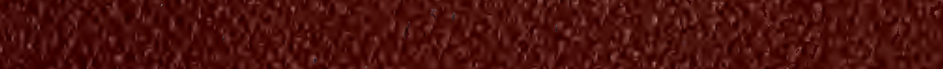

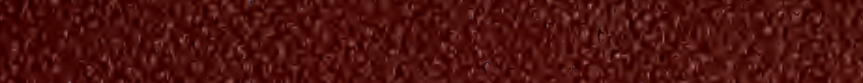

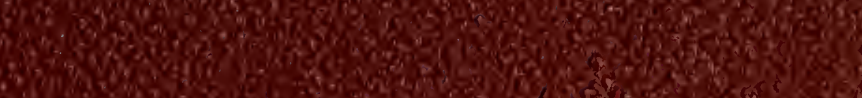

S.

5. 4..0.

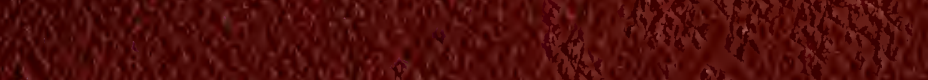
3iv

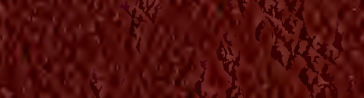
13.

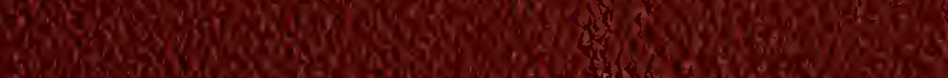

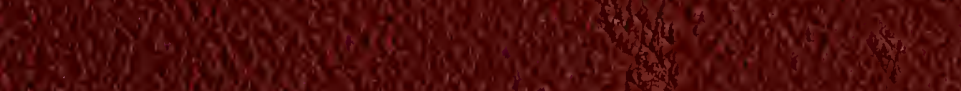

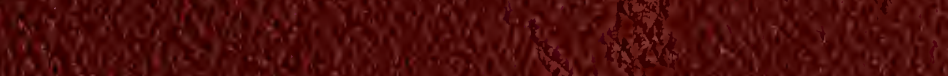
1).

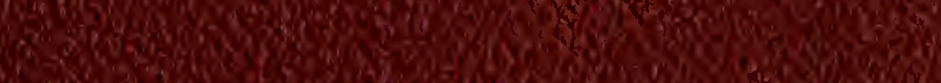
ans 109 3 ang

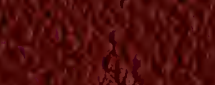

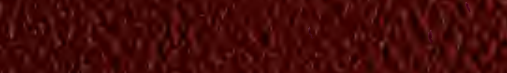
19 ying $4 \tan ^{2}$ Q.) I. 4)

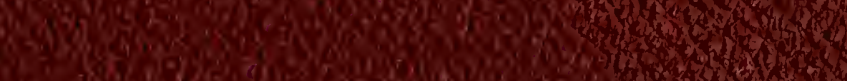
250. 


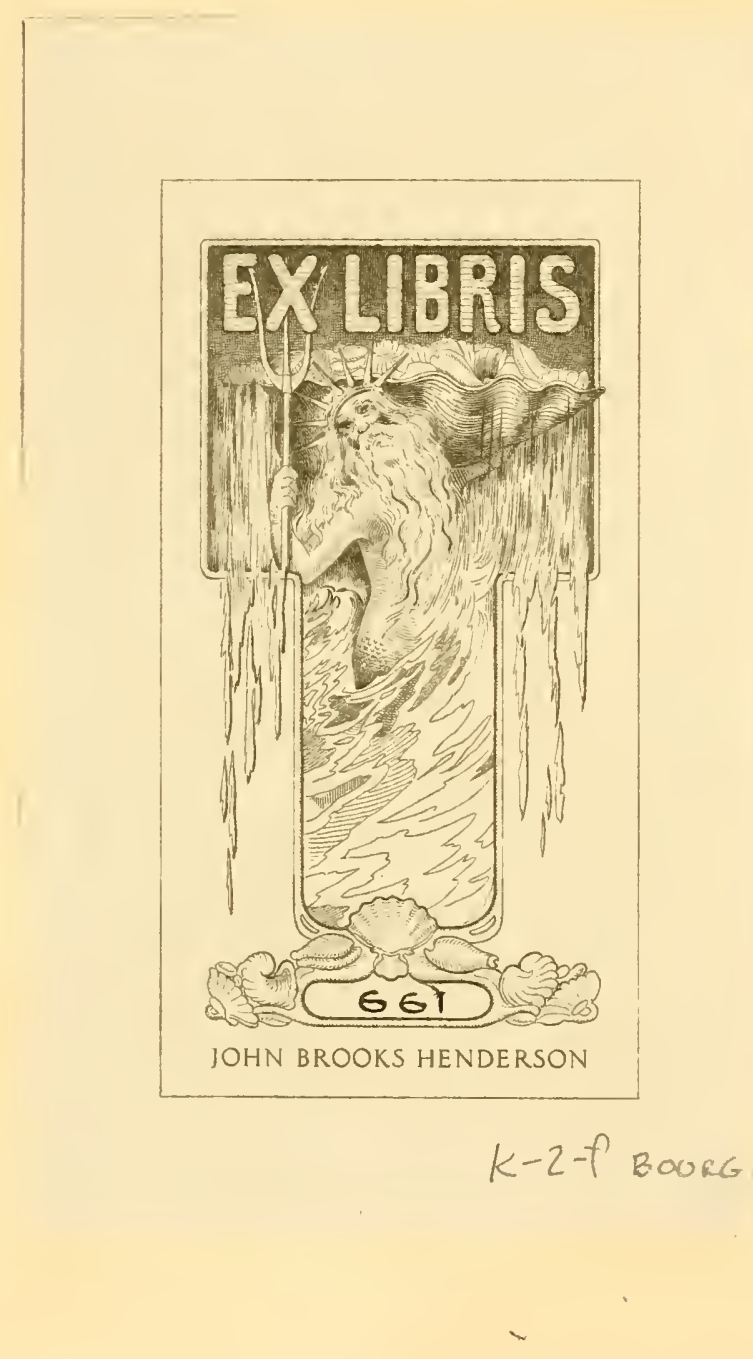

GIAU WESLEY \& SOV, WADI WESLE 




\section{MOLLUSQUES}

D E

\section{L'AFRIQUE EQUUTORIALE}





\section{MOLLUSQUES}

DE

\section{LAFRIOQUE ÉQUATORIALE}

DE NOGUEDOUCHOU A BAGAMOYO

ET DE

BAGAMOYO AU TANGANIKA

M. J. R. ${ }_{i l}^{\text {BOURGUignat }}$

SECRÉtaIRE GÉNÉRAL DE LA SOCIÉtÉ MaLACOLOGIQUe

DE FRANCE, ETC.

SWIt RGGWIN

MAY 201988

\section{LIBAARIES}

PARIS

IMPRIMERIE D. DUMOULIN ET Cie

5, RUE DES GRANDS-AUGUSTINS, :3

MARS 1889

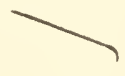



J'ai eu, longtemps, l'intention de donner une Histoire malacologique des régions équatoriales de l'Afrique. Aux documents assez nombreux, parvenus entre mes mains, j'espérais, en y adjoignant les renseignements publiés par les auteurs, mes devanciers, pouvoir arriver à présenter une faune a peu près complète de ces vastes contrées, lorsqu'après réflexions et examen du nombre relativement petit de documents, comparativement à celui plus considérable que les explorations des immenses espaces encore inconnus auraient pu me fournir, je compris que le plan que je m'étais proposé n'était pas réalisable.

Le moment n'est pas venu de publier une faune de ces régions; ce ne sera guère que dans une vingtaine d'aunces que l'on pourra entreprendre un pareil travail.

En attendant, j'ai pensé qu'il serait peut-être utile de mettre en lumière les renseignements à ma connaisssance, dans le but d'apporter une pierre à l'édification de la future faune équatoriale.

Ce sont done ces renseignements que je présente actuellement, renseignements essentiellement malacologiques, consacrés aux Espèces re- 
cueillies dans les immenses territoires qui, des côtes de l'océan Indien, s'étendent jusqu'à celles qui circonscrivent le grand lac Tanganika, c'esta-dire sur un espace de pays égalant à vol d'oiseau plus que la longueur de la France entière, du Nord au Midi.

Quelques mols sur cette vaste étendue de pays sont nécessaires pour l'intelligence du clamp malacologique de cet ourrage.

Deux bassins, celui du Kyngani el celui du Vouami, occupent les régions en face du canal de Zanzibar, régions aujourd'hui sous la domination allemande.

Le bassin du Kyngani, an sud de Bagamoyo, limité à l'Occident par une chaine de hautes collines, comprend les territoires Ouzaramo, Oukonéré, Ouseguoa, Ourougourou et Oukami. Le Kyngani, dont le plus fort aflluent est le Guéringuéré, arrose tous ces pays.

Le bassin du Vonami, situé plus au Nord, se déreloppe depuis Sadani, petit port à son embouchure, jusqu’a l'Ougogo, dont il n'est séparé que par une chaine montueuse. Toute la partie supélieure de ce bassin forme le pays de l'Ousaghara, où se montrait naguc̀re la station hospitalière de Kondoa, fondée par notre compatriote, le capitaine Bloyet.

Au nord de ce bassin se dresse le massif montueux du N"gourou. dont les plus hautes cimes alfoignent 2500 metres. C'est sur un de ses contre- 
forts, à M'hounda, que s'élèvent les bâtiments de la mission française des RR. PP. missionnaires du Saint-Esprit.

Au-delà de l'Ousaghara, entre deux chaînes de montagnes, s'étend, sur une largeur de 160 kilomètres, l'Ougogo, pays très peuplé, arrosé par le Dendalasongoué, affluent du Roufidgi, qui porte ses eaux à l'océan Indien, en face l'ìle Mafia, au nord de Quiloa.

Au sortir de l'Ougogo, l'on entre sur le territoire des Ouyanzi et des Ourimbou, territoire presque entièrement recouvert par l'immense forêt "M'gounda-Mkali ", sorte de maquis souvent impénétrable, tantôt aride, tantôt marécageux, pour parvenir, après l'avoir traversée, à la région des Ounyamouezi, dont la plus importante peuplade, celle de l'Ounyanyembé, a pour ville principale Tabora', point central d'ou partent les caravanes, soit pour le Tanganika, soit pour les Nyanzas nilotiques.

De Tabora, en se dirigeant tonjours dans la direction de l'Ouest, on traverse de nombreuses campagnes cultivées, puis une vaste forèt, aux arbres magnifiques, pour tomber dans le bassin du Malagarazi, la plus forte rivière tributaire du Tanganika, qui, à quarante kilomètres au-dessous d'Oudjiji, va se perdre dans le lac.

Le lac Tanganika, où l'on parvient enfin, après une route de plus de 1400 kilomètres, occupe une

1. C'est à dix kilomètres de celte ville que se trouve la Mission française de Kipalapala des RR. PP. missionnaires. 
vaste dépression s'étendant, du Nord au Sud, sur une longueur de plus de 600 kilomètres.

C'est sur ses côtes, dont le développement dépasse 1500 kilomètres, côtes occupées par de nombreuses peuplades, que se trouvent Oudjiji, Karéma, Pambété, ete., les missions de Kibanga, Mpala, Mlilo, etc., où ont été recueillis les nombreux Mollusques d'eau douce (271 Espèces), que j'ai fait connaitre dans mon Histoire malacologique du lac Tanganita.

Dans le travail actuel, j'ai laissé de côté toutes les Espèces fhuviatiles du lac; je n'ai publié dans cet ouvrage, complément naturel de celui du Tanganika, que les formes découvertes dans les pays du pourtour ou dans les cours d'eau qui les arrosent.

- Les documents malacologiques que je fais connaitre sont donc ceux relatifs aux territoires que je viens de mentionner.

A ces documents, j'ai cru devoir joindre ceux qui me sont parvenus de toute la côte zanguébarienne, depuis Moguedouchou jusqu'à Bagamoyo.

Je dois la connaissance des Espèces de la côte orientale, d'abord à notre excellent ami le conseiller Letourneux, puis au courageux explorateur du pays Comalis, M. Georges Revoil ${ }^{1}$, qui, au péril de ses jours, remonta le cours de l'Ouebi jusqu'audelà de Guélidi; enfin, à plusieurs voyageurs et au R. P. Alexandre Leroy, de la Mission de Zanzibar.

1. Voyage chez les Bénadirs, les Comalis et les Bayouns, in : le Tour du Nonde, 1885. 
Je dois celle des Espèces de l'intérient : $1^{\circ}$ it l'intrépide voyageur, le lieutenant de marine, M. Victor Giraud, qui, après sa périlleuse expédition au lac Bangouélo, visita les còtes méridionales du Tanganika, avant d'effectuer son retour par le Nyassa et le Shiré; 2" au capitaine Bloyet, le fondateur de la station de Kondoa; $3^{\circ}$ au capitaine Léopold Joubert, actuellement commandant les forees militaires du Tanganika; enfin, $4^{\circ}$ à plusieurs Pères missionnaires qui, dans leur modestie. désirent garder l'incognito.

Toutes les découvertes dues à ces zélés voyageurs, explorateurs et missionnaires, auxquels, avec mes remerciements, s'adresse l'expression de ma reconnaissance, si elles ont fini, en s'accumulant, par former un ensemble scientifique assez important pour l'histoire malacologique de ces régions, elles ne sont pas néanmoins suflisantes pour constituer la base d'une faune générale.

C'est pour ce motif, que laissant de còté mon idée première, je me permets de les présenter seulement sous forme de chapitres indépendants les uns des autres.

$$
\text { J.-R. B. }
$$

Saint-Germain, 15 févier 1889. 



\section{MOLLUSQUES}

\section{E \\ L'AFRIQUE ÉQUATORIALE}

I

\section{HELIXARIONIDE}

Je comprends dans celte famille toutes les Espèces Hyaliniforme ou Héliciforme, dont les Animaux ont l'extrémité du pied netlement tronquée et pourvue d'un large pore muqueux.

Je laisse dans la nounelle famille des Africarionidie 1 toutes les autres à test vilriniforme, pour lesquelles Godwin-Austen ${ }^{2}$, avec raison, a ćlabli sa coupe générique d'Afiricarion, pour la pallens ${ }^{3}$ de l'Abyssinic .

Le genre OErope ${ }^{5}$, créé pour l'Helix caffir ${ }^{6}$, doit également faire partie de celte famille ${ }^{7}$.

1. Bourguignat, in sclied., 1888.

2. Moll. ind., p. 15 't et 163 (oct. 1883).

3. Helicarion pallens, Morclet, in : Ann, mus. civ. Genora, III, 1872, p. 189, pl. w, fig. 4.

4. Les autres Africarions sont les lymphaseus (Morelet, 1872), Raffrayi (Bourguignat, 1883), Ragazzii (Pollonera, 1887), semi-membranaccus et plicalulus (Marlens, 1876), d'Abyssinie, du Choa et du Gabon.

5. Albers, Helic., p. 83, 1860.

6. Férussac, Prodr., 1821, et Hist. moll., pl. Ix ${ }^{A}$, fig. 8.

7. L'Helix Wesseliana (Malzan, in : Jahrb. mal. Gesellsch., 18:6, p. 149, pl. v, fig. 1 l est une Erope. 
Les Ilélixarionidées, réduites anx Espéces a lest Hyaliniforme on Héliciforme, comprennent les genres suivants :

\section{TAPSI A}

Tapsia, Ilbers, Ilelic., p. 56, 1860.

Ce genre, établi aux dépens des Nanina d'Asie, pour des Espèces africaines /yalinoüdes, est composé de formes ressemblant beaucoup à certaines Iyalinies d'Europe, de la série des Lucida, septenIrionalis, ete. Parmi les formes de ce genre, je mentionnerai les egenula (Helix), columellaris (Helix), Pfeiffer, du Sénégal; - pellucida, indecorata et cerea (Helix), Gould, Thomensis (Nanina) Dehrn; Liberix (IJelix) Brown; chrysosticta et lroglodyles (Helix), Morelet; glomus (Nanina), Albers; calamechroa (Helix), Jonas; Buchholzi, (Tapsia), Bourguignat, ete..., des côtes de Guinće, du Gabon et des ìles voisines de ces régions; oleosa (Helix), Pfeiffer ; emryomphala (Tapsia), Bourguignal; abyssinica et Vesti (Hyalinia), Jickeli, de l'Abyssinie et du Choa.

Je ne connais qu'une Tapsie a signaler du Zanguébar1, la Tapsia Leroyi (Grandidier, in : Bull. Soc. malac. Fr., IV, 1887, p. 186).

1. C'est par suite d'une erreur de géographie que dans mes Ilélixarionidées des régions orientales de l'Afrique (Paris, in-8, 1885), j'ii confondu le Bonjongo des monts Cameroon, menlionné par le doctenr Marlens, aree un autre Bonjongo du Mozambique; erreur qui na a fait sigualer, at tor, dans cette ré" 1 , plusieurs Espèces, qui n'y virent pas. 
Cette Espèce qui, comme forme et comme aspect, ressemble beancoup a la Tapsia calamechroa ${ }^{1}$ des monts Cameroon (Gabon), se disting'ue néanmoins de cette Tapsie, telle qu'elle est représentée par le Dr Martens, par sa spire moins convexe, par son onverture plus échancrée, phus en forme de croissant, et par son dernier tour dont le maximum de la convexité est plus supérieur.

Environs du couvent des RR. PP. du Saint-Esprit, près de Bagamoyo.

\section{SIT A L A}

Sitala, H. Adams, in : Proc. Zool. Soc. Lond., 1856, p. 408.

Ce genre a été également établi, atıx dépens des Nanina, pour de très petites Espèces brillantes, transparentes, ressemblant aux Conulus d'Europe.

Les Sitales africaines sont les: membranacea (IIelix) Jickeli; Raffrayi (Sitala), Bourguignat; Trochulus (Sitala), Pollonera, des régions abyssiniennes ${ }^{2}$. Je n'en connais pas des contrées équatoriales.

1. Bourguignat, 1884, et Hélixarionidées, p. 6, 1885 (Helix tenera, Jonas, in : Philippi, 1843, et Nanina calamechroa, Vartens, Buccholz moll., p. 254, pl. 1, fig. 10-11, 1876.

2. Pour la synonymie de ces Espèces voir ma "Malacologie de l'Abyssinie ", 1883, et le mémoire "Nuove specie di moll. err. raccolti nello Scioa, fév. 1888, du docteur C. Pollonera. 


\section{MOARIA}

Moaria, Chaper, in : Bull. Soc. zool. Fr., 1885.

Ce genre a été créé pour une Espèce des côtes de Guinée, la Moaria conica ${ }^{1}$, remarquable par sa forme trochoüde et par les sillons spiraux qui ornent la partie supérieure des tours.

Il convient de rapporter à cette coupe générique les Helix talcosa ${ }^{2}$ et calabarica ${ }^{3}$ des còtes de Guinée et du Gabon.

Le R. P. Leroy a découvert, dans les parties humides et boisées du N'gourou, au nord de l'Ousaghara, une minuscule Espèce de ce genre.

\section{MOARIA CHAPERIANA}

Testa minuta, anguste perforata, conoïdea, fragili, pellucida, cornea, subtus lavigata, supra spiraliter substriatula; - spira sat breviter conica, nihilominus ad summum obtusiuscula ; - anfractibus 6 tectiformibus, vix subconvexiusculis, lente crescentibus, sutura parum impressula separatis; - ultimo obscure subangulato, ad insertionem breviter subdescendente; - apertura subobliqua, parum lunata, irregulariter transverse oblonga;-peristomate recto, acuto, inferne leviter

1. Chaper, Esp. et g. nouv. (ext. Bull. Soc. zool. Fr., X, 1885), p. 8, pl. 1, fig. 12 .

2. Gould, in : Proc. Bost. Soc., III, 1850, p. 194, el Pfeiffer, Novitates, pl. crint, fig. 7-9.

3. Pfeiffer, in : Proc. zool. Soc. Lond., p. 327, et Novitates, pl. crin, fig. 10-12. - Espèce très déprimée, peu conique. 
subpatulo ac incrassatulo; margine columellari ad summum triangulatim expanso; - alt. ac diam. reque 2 ; alt. ap. 1, lat. $11 / 4$ millim.

Cette très petite Espèce, à laquelle j'attribue le nom du créateur du genre, M. Maurice Chaper, se distingue de sa congénère d'Assinie par sa taille bien plus petite, puisqu'elle a 2 millim., tandis que la conica en a 8, par ses sillons spiraux moins prononcés, par son demier tour moins anguleux et par son ouverture de forme différente.

Les Espèces de ce genre ont un aspect tout particulier qui ne permet pas de les confondre ni arec les Sitales, ni avec les Trochonanines; on les prendrait plutôt pour le sommet d'une coquille inconnue, si l'on ne r'emarquait pas, chez elles, une légère direction descendante à l'endroit de l'insertion du bord externe, signe certain de leur état adulte.

\section{TROCHONANINA}

Trochonanina, Molzsson, in : Journ. Conch., 1869, p. 330 .

Ce genre a élé établi, aux dépens des Trochomorpha, pour des Espèces conico-carénées, à test mince, assez transparent, strié ou lamellé en dessus, presque lisse en dessous, et à péristome seulement réfléchi triangulairement au sommet columellaire.

Les Trochonanines sont des formes océaniennes que l'on rencontre aux îles Marquises, Bornéo, 
Java, Sumatra, Manrice, Bourbon, ainsi que dans la presqu’ìle de Malacca.

En Afrique, les Espèces de ce genre ont été constatées dans les contrées occidentales ${ }^{1}$, voisines des côtes, ainsi que dans quelques îles limitrophes de ces côtes, et, dans les régions orientales, au Mozambique, à Zanzibar et au Zanguébar. Seulement, dans cette dernic̀re région, elles ne paraissent pas dépasser le niveau de Monbas, oì, plus au Nord, elles semblent être remplacées par les Ledoulxies.

Les vraies Trochonanines sont les suivantes:

Trochonanina Folisi², Bourguignat (Helix Folini, Morelet, in : Rev. zool., 1848, p. 352, et Sér. conch., I, 1858, p. 13, pl. 1, fig. 3.-Trochomorpha Folini, Pfeiffer, Nomencl., p. 80, 1878). - Belle Espèce hispide. - lle du Prince (côte du Gabon).

Trochonanina Adansonie, Bollguignat (Helix Adansonix, Morelet, in : Rev. zool., p. 351, et Sér. conch., I, 1858, p. 13, pl. I, fig. 4.-Conulus Adansonia, Pfeiffer, Nomencl., p. 74, 1878). Gabou.

Trochoxaxixa agupta, Bourguignat (Nanina aglypta, Martens, in : Malak. BI., 1866, p. 119 , pl. v, fig. 5-7). - Ile du Prince.

Trochonasixa trumula, Marters, Buchholz,

1. Gabon, Guinée, Benguella.

2. Ancey (Bull. soc. malac. Fr., V, 1888) place cette Espèce, ainsi que la suivante, l'Adansonix, dans le genre trochozonites de Pfeiffer, genre qui m'est inconnu. 
moll. in Monatsb. Wiss. Berl., 1876, p. 256, pl. I, fig. 12-14. - Gabon (monts Caméroon).

Trochonaxina plicatula, Pfeiffer, Nomencl., 1878, p. 57 (Nanina plicatula, Martens, conch. Zanzib. Sesamsaanen, in : Nachr. Malak. ges., 1869, p. 149; Helix plicatula, Pfeiffer, Monogr. Hel., VII, 1876, p. 97, et Nanina plicatula, Pfeiffer, novitates, IV, 1876, p. 48, pl. cxvin, fig. 17-18). Ile de Zanzibar.

Trocionaxisa Nrassana, Bourguignat (Helix [nanina] Nyassana, Smith, in : Proc. zool. Soc. Lond., 1881, p. 278, pl. xxxir, fig. 2, $2^{\mathrm{A}}$ et $2^{\mathrm{B}}$ ). Grande Espèce des contrées entre le Nyassa et l'Océan.

Trochonasixa Smith, Bourguignat (Helix [Trochonanina] Mozambicensis, Smith, in : Proc. zool. Soc. Lond., 1881, p. 279, pl. xxxı, fig. $3-3^{\mathrm{A}}$ ). Mème localité que la précédente. Cette Espèce, à laquelle j'attribue le nom du docteur Smith, est tont à fait dissemblable de la vraie Mozambicensis. La Smithi est une forme déprimée également très différente des Trochonanina Anceyi, Bloyeti, percarinata et autres.

Les Trochonanines qui suivent ont toutes été constatées dans les régions qui constituent le champ malacologique de cet ouvrage.

Trochomaxina Mozambicensis, Mousson, in : Journ. conch., 1869, p. 330 (Helix Mozambicensis, Pfeiffer, in : P'roc. rool. Soc. Lond. 1855, p. 91. 
pl. xxxi, fig. 9. - Martensia Mozambicensis, Semper, Phill. Land moll. p. 42, $1870^{1}$ ). - La Mozambicensis est une petite coquille très voisine, comme forme, de l'Ibuensis, dont elle ne diffère guère que par son dernier tour, plus convexe en dessous. La Trochonanina Mossambicensis, var. de Martens (Moll. Decken, p. 56, pl. I, fig. 2, et Hildebrandt, Conch., p. 289, 1878) est la Ledoulxia albopicta. Le type de la Mozambicensis provient de Tette, sur le Zambèse (Mozambique). - Environs de Kondoa (Ousaghara).

Trocionanina Ibuexsis, Martens, Buchholz Moll., in : Monatsb. Wiss., Berl., 1876, p. 256, pl. I, fig. 15 (Helix Ibuensis, Pfeiffer, Symb. Hel., 11, p. 66, 1846). - Ibu (Mozambique). Vallée du Kyngani, sur le territoire des Okami. - Cette Espèce se rencontre assez fréquemment dans les sacs de Sésame provenant du Zanguébar.

TROCHONANINA LEROYT, spec. nov. (pl. i, fig. 13-14)

Testa vix punctiforme subperforata, conica (conus leviter concavus), percarinata (carina suturam superficialem sequens, in ultimo producta ac strangulato-acuta), tenui, subtranslucida, uniformiter corneo-lutescente, supra striatula ac parum nitente, subtus quasi polita ac splendide nitidissima; spira conico-tectiformi, ad summum acutiuscula

1. Semper, se basant sur quelques détails du collier et sur quelques particularités de l'appareil reproducleur, a établi le nouveau nom de Marénsia, nom qui a élé adoplé par Jickeli (Moll. N. O. Afr., p. 50, 187i). 
(apex exiguus); - anfractibus 8 acute carinatis, tectiforme planis, lente crescentibus; - ultimo majore, compresso, percarinato (carina peracuta, producta), supra tectiformi, infra convexiusculo; - apertura parum obliqua ac lunata, irregulariter transverse suboblonga, ad carinam canaliculata; - peristomate fragili, acuto, recto; margine columellari superne reflexo ac triangulatim dilatato; - alt., 11; diam., 7; alt. ap., 4; lat., 8 millim.

Cette Espèce, remarquable par sa forme conique, similaire à celle d'un chapeau chinois, ne peut être assimilée avec aucune de ses congénères. Elle a été recueillie par le R. P. Leroy, à qui elle est dédiée, dans les endroits ombreux des monts N'gourou, au nord de l'Ousaghara.

Trochonaxixi Jexmsi, Martens, Hildebrand, Conch., in : Monatsb. Wiss., Berl., 1878, p. 290 (Helix Jenynsi, Pfeiffer, in : Proc. zool. Soc., Lond., 1845, p. 131, et [2 édit., Chemnitz] n ${ }^{\circ} 821$, pl. cxxix, fig. 23-24; Nanina Jenynsi, Gray, 1855, et Martens, 1869, etc.). - Cette forme cosmopolite a été retrouvée aux environs de Bagamoyo, de Kiondoa (Ousaghara) et de Monbas.

Trochonaniva percaninata, Martens, Buchholz Moll., in : Monatsb. Wiss., Berl., 1876, p. 256, pl. , fig. 16-18. - Cette Espèce, du Gabon (mont Cameroon), a été constatée fréquemment dans le Mozambique et le Zanguébar. C'est la forme la plus commune que l'on trouve dans les sacs de 
Sésame provenant de ces régions. Elle a été recueillie, dans ces derniers temps, dans l'ile de Zanzibar, ainsi que presque sur toute la còte africaine à Dar-ès-Salam, Bagamoyo, Pangani, Tanga et Monbas. Je ne la connais pas de l'intérieur.

Trochonaxixa Axceyt, Bourguignat, Hélixar. Af., p. 9, 1885.

Petite Espèce (haut., 7; diam., 12 millim.), discoïde, déprimée, presque aussi convexe en dessus qu'en dessous, pourvue d'une perforation réduite à un point. Test brillant, presque opaque, très délicatement striolé en dessus, poli et présentant en dessous de nombreuses petites linéoles spirales. Coloration d'un roux uniforme avec des taches plus pâles passant, en dessous, à une teinte d'un ton de café au lait, orné, çà et là, de petites taches ponctiformes d'une nuance marron. Spire déprimée, conoïde, peu laute, à sommet aigu surmonté par un tour embryonnaire minuscule et proéminent. Six tours peu convexes, à croissance régulière, séparés par une suture un tant soit peu accentuée. Dernier tour de taille médiocre, à carène aiguë, peu convexe en dessus, plus convexe en dessous. Ouverture peu oblique, échancrée, d'une forme semi-oblongue, dans le sens transversal, avec une angulosité marquée à l'endroit de la carène. Péristome droit, aigu, un tant soit peu épaissi à l'intérieur, faiblement subpatulescent inférieurement et offrant, à la partie supérieure du bord colımellaire, une courte dilatation triangulaire. 
Cette Espèce, découverte primitivement dans des sacs de Sésame provenant du Zangtiébar, a été retrouvée vivante aux environs de Kondoa (Ousaghara).

TROCHONANINA BLOYETI, spec. nov. (pl. II, fig. 10-12).

Testa aperte profundeque perforata, lenticulari, depressa, supra infraque fere aqualiter convexa, solidula, opacula, mediocriter nitente, cornea, eleganter valide striata (stria, quasi lamellosx, regulares, obliqua ac producte, et, circa suturam albo-punctata, subtus pallidiore, nitidiore et quasi polita ; - spira subtectiforme depressa, perobtusa subconoidea, ad summum rotundata; - anfractibus vix subconvexiusculis, regulariter crescentibus, earinatis (carina suturam leviter impressulam sequens); - ultimo majore, carinato (carina acuta), supra parum convexiusculo, subtus convexiore; - apertura obliqua, sat lunata, transverse irregulariter oblonga, ad carinam angulata; - pe ristomate recto, subincrassatulo, inferne leviter patulescente; margine columellari superne mediocriter dilatato; - alt., 7 ; diam., 15; alt. ap., 4; lat., 7 millim.

Cette Espèce, remarquable par sá dépression spirale, par sa large perforation, par ses striations lamelliformes munies, le long de la suture, de points saillants similaires aux costulations albosuturales des Clausilies européennes de la série de la $\mathrm{Cl}$. punctata, est très distincte des Trocho- 
nanina Smithi et Anceyi, les seules qui, par leur taille et l'ensemble de leur forme, peuvent lui être assimilées.

Environs de Kondoa(Ousaghara), où elle a été recueillie par le capitaine Bloyet.

\section{ZINGIS}

Zingis, Martens, in : Monatsb. Wiss. Berl., 1878, p. 290 .

Ce genre a pour type une coquille mince, hyalinoïde, la Zingis radiolata ${ }^{1}$ de Zanzibar, remarquable par sa croissance spirale très rapide, par son dernier tour très développé, par une spire brièvement conoïde, par une ouverture très ample, subarrondie, et par un péristome délicat, tranchant, dilaté seulement triangulairement au sommet columellaire.

D'après la figure de l'appareil reproducteur donné par le $\mathrm{D}^{\mathrm{r}}$ Martens ${ }^{2}$, le fourreau de la verge na pas de flagellum, le vagin ressemble à un sac ovoïde, à l'extrémité duquel se trouve un court oviducte et d'où part, à son origine, l'appareil de la poche copulatrice, biparti, d'un côté, en une branche terminée par une glande oblongue, et, de l'autre, en un conduit en cul-de-sac. Ce genre, d'après les caractères de son appareil reproducteur, tient des Hyalinia et des Helix.

1. Martens, Hildebrandt, Conch. in : Monatsb. Wiss. Berl., $18 \% 8$, p. 290 , pl. I, fig. 8-17.

2. Monatsb. Wiss. Berlin, 1878, pl. s, fig. 11. 
H A M A

Hamya, Bourguignat, Hélixar. Afr., p. 11, 1885.

Ce genre est caractérisé par une coquille hyalinoïde, offrant sur son dernier tour, à 4 millimètres en avant de l'ouverture, un étranglement analogue a celui qui distingue certains genres de Cyclostomidés, tel que celui des Alycxus. A partir de cet étranglement, d'une étendne de 3 millimètres, le tour reprend sa forme et son volume pour se contracter de nonvean, mais d'une facon moins prononcée, seulement à la partie supérieure du bord péristomal.

Une seule Espèce connue des dépôts quaternaires de l'Ouébi, entre Meurka et Moguedouchou, ou elle a été déconverte par le voyageur G. Revoil.

Hamy Revolu, Bourguignat, Hélixar. Afr., p.11, 1885.

Coq. étroitement perforée, hyaliniforme, fragile, finement striolée, déprimée, offrant, en dessous, une surface convexe avec une concavité assez prononcée à son centre, et, en dessus, une surface plus convexe. Six tours médiocrement bombés, à croissance lente, séparés par une suture accentuée. Dernier tour comprimé tout en restant presque rond, subanguleux à son origine, brièvement descendant à l'insertion, offrant, en avant de l'ouverture, un large et profond étranglement et une con. traction à la partie supérieure du bord péristomal. Ouverture oblique, échancrée, semi-oblongue dans 
le sens transversal, entourée par un péristome aigu, rectiligne, légèrement infléchi à sa partie supérieure, faiblement patulescent à la base et offrant, au sommet du bord columellaire, une dilatation triangulaire. Haut., 8; diam., 14 millim.

\section{LEDOULXIA}

Ledoulxia, Bourguignat, Hélixar., rég. orient. Afr. - Janv. 1885, p. 12.

Ce genre, dédié à M. Ledoulx, ancien consul de France, à Zanzibar, se compose d'Espèces subtrochoïdes, à test opaque plus fortement strié en dessus qu'en dessous et ordinairement d'un blanc uniforme, à ouverture échancrée, transversalement oblongue et entourée par un bord péristomal aigu, parfois obtus et fortement bordé.

Les Ledoulxies paraissent s'étendre depuis la latitude de Zanzibar jusqu'en Abyssinie et à la terre d'Adel; elles remplacent dans ces régions les vraies Trochonanines dont l'aréa semble plus méridional.

A l'exception de la Ledoulxia Alfieriana', du Choa, toutes les autres ont été trouvées de Moquedouchou à Bagamoyo.

Ledovlxia albopicta, Bourg., Hélixar., p. 12, 1885 (Nanina Mozambicensis, var. albopicta, Martens, Moll. Decken, p. 56., pl. I., fig. 2, 1869). Cetteforme, que le $\mathrm{D}^{\mathrm{r}}$ Martens, à tort, a assimilée au Mozambi-

1. Soleillet, in : Bourguignat, Họlixar., p. 13, 1885, et Moll. Choa, p. 7, 1885 . 
censis de Pfeiffer, se trouve aux enviroms de Monbas, ainsi qu'au cap Gassi entre Mombas et Tanga. Les échantillons de cette localité sont plus blancs et plus opaques que ceux de Monbas.

Ledoulxia pyranidea. Bourg. Hélixar., p. 12 , 1885 (Nanina pyramidaea, Martens, Moll. Decken, p. 55, pl. I, f. 3, 1869, et Trochonanina pyramidaea, Martens, Hildebrandt, Conch. in. Monatsb. Wiss. Berl. 1878, p. 289, pl. I, fig. 5-7).

Espèce à spire plus conoïde, à test plus opaque, au dernier tour moins anguleux que la précédente. Guélidi, à trois journées de marche de Moguedouchou, environs de Monbas; Tanga, chez les Ousambura; Kitoni chez les Oukamba, au nord de l'Ousaghara'.

Ledoulxia formosa, Bourguignat, Hélixar., p. 14, 1885.

Environs de Guélidi, près Moguedonchou. Cette Espece se distingue de la Ledoulxia pyramidxa, telle qu'elle a été figurée par le $\mathrm{D}^{\mathrm{r}}$ Martens, par sa taille moindre, par sa forme plus déprimée, par sa spire un peu moins conoïde, par sa croissance spirale plus serrée, par son dernier tour plus exigu, moins gros, par son ouverture semi-oblongue dans un sens transversal faiblement incliné de ganche à droite, entourée par un bord péristomal épaissi a l'intérieur, notamment au bord inférieur.

Le dessous du dernier tour se creuse sensible-

1. Cette Ledoulxie a été également constatéc dans le Choa, (Bourguignal, Moll. Choa, p. T, 1885.) 
ment vers la perforation et parait légèrement concave, ce qui n'a pas lieu ni chezl'Alfieriana, ni chez la pyramidixa.

Ledoulxia megastona, Bourguignat, Hélixar., p. 14, 1885.

Cette Ledoulxie, de même taille que la formosa, est caractérisée par un dernier tour très renfléconvexe en dessous, et par une grande ouverture exactement hémisphérique et un peu en disproportion avee la taille générale. Haut. ouv., 7 1/2; larg., 8; hauteur du test, 10; diam., 15 millim.

Chez cette Espèce, également très costulée en dessus et plus polie-brillante en dessous, le dernier tour, d'abord comprimé et anguleux à son origine, finit, en grossissant rapidement, par devenir presque rond à l'ouverture. - Environs de Guélidi.

Ledoulxia insıgnis, Bourguignat, Hélixar., p. 15, 1885.

Espèce subglobuleuse (Haut., 11; diam., 17 millim.), non anguleuse, sauf un tant soit peu à l'origine du dernier tour. Test opaque, blanchâtre, avec des vestiges de zonules très étroites, interrompues et fort peu apparentes en dessous. Costulations fines, très serrées en dessus. Sept tours assez convexes, à croissance lente et régulière, séparés par une suture bien accentuée. Dernier tour subcomprimé-arrondi, légèrement et lentement dèscendant a l'insertion. Ouverture échancrée, semi-ovalaire, a péristome aigu, tranchant au bord supérieur, 
épais, obtus et patulescent au bord inférieur et à peine dilaté au bord columellaire.

L'encrassement interne péristomal, qui est marginal à la base aperturale, se montre très enfoncé supérieurement par suite de la projection en avant du bord externe, sous l'apparence d'une paroi mince et tranchante.

Vallée de l'Ouébi, entre Guélidi et Mognedouchou.

Ledoulxia uxizonata, Bourguignal, Hélixar., p. 15, 1885 .

Cette Espèce, remarquable par sa belle zone étroite d'un noir-marron intense, se détachant sur un fond blanchàtre tirant légèrement sur une nuance jaunacée, ressemble, au point de vue de la coloration, aux Campylées alpiques d'Europe, telles que les phalerata, glacialis et cingulata.

Chez la Ledoulxia unizonata, le test opaque est aussi finement strié en dessus qu'en dessous; l'ombilic est relativement assez ouvert; la spiro (de 7 tours), à croissance lente, est obtusément conoïde; le dernier tour, faiblement et brièvement descendant à l'insertion, est comprimé-subarrondi sans angulosité; l'ouverture, très échancrée semioblongue, est entourée par un bord péristomal aigu, intérieurement bordé et extérieurement patulescent.

Environs de Gućlidi, près Moguedouchou. 


\section{BLOYETIA}

(Olim Guillainia, Bourguignät, Hélixarionidées, p. 16, Jano. 1885! - non Guillainia, Crasse in : Journ. conch., p. $370^{1}$, dans le numéro portant la date d'octobre 1884, tandis qu'eu réalité ce numéro n'a paru que le 15 avril 1885?, c'est-ì-dire plus de deux mois aprés la publication de mes Hélixarionidées.)

Malgré l'évidence de mon droit à l'antériorité du nom générique de Guillainia, j’aime mieux, pour éviter aux malacologistes futırs toute confusion, laisser de côté ce nom pour prendre celıi du brave capitaine Bloyet, le fondateur de la station de Kondoa (Ousaghara).

Ce genre Bloyetia (olim Guillainia) est composé d'Espèces de toute beauté. Ces Espèces, an nombre de huit, découvertes par le voyageur M. G. Revoil, dans sa dernière exploration ${ }^{3}$, proviennent toutes de la vallée de l'Ouébi ${ }^{4}$ et des environs de Guélidi,

1. Mauvais nom générique établi pour la Rochebrunia tricolor (Bourguignat, Moll. Revoil, p. 83, 1882), Espèce de Cyclostomidé de l'ile de Socotora.

2. Comme preuve, je possède le bon à tirer de la feuille 25 du Journal de conehyliologie (comprenant les pages 357 à 372 , oin se troure le genre Guillainia), indiquant, pour date de tirage " 25 mars 1885, 385 ex., signé André ", ainsi que le bon a tirer de la couverture de ce numéro d'octobre 1884. Ce bon porte comme date du tirage, celle du " 4 arril 1885 »!

3. Voyage ehez les Bénadirs, les Comalis et les Bayouns, in : Tour du Monde, 1885.

4. L'Ouébi, un des plus grands cours d’eau des régions ço- 
à quatre ì cinc jours de marche de Moguedouchou. le Magadoxo des Portugais.

Les Bloyeties sont des animaux nocturnes, elles se terrent, le jour, dans les broussailles, sous les pierres ou dans le sable.

Les deux Bloyeties, la Mabilliana et la Leroyi, paraissent des formes disparues; elies n'ont été découvertes, jusqu'à présent, que dans les dépôts sablonneux de la vallée oućbienne.

Les Espèces de ce genre, caractérisées par un test calcaire, pesant, opaque, fortement strié ou costulé, d'une forme globuleuse, à spire conoïde et à péristome obtıs, sont remarquables par leur coloration bleuâtre et par l'intérieur de leur bouche d'une teinte intense noir-marron, analogue a celle que l'on observe chez l'Hélix Lucasi, d'Algérie.

L'animal est jaunâtre, avec un collier d'un beau noir-ardoisé. La màchoire et le ruban lingual présentent une disposition à peu près analogue à celle des Hyalinies européennes.

L'appareil reproductem (pl. 1, fig. 15') offre une glande copulatrice volumineuse, un fourreau de la verge étranglé à sa partie moyenne et se prolongeant, à partir de l'insertion du canal déférent,

malienues, a son origine aux environs d'Harrar, au sud du massif éthiopien; après un long parcours peu connu, dans le pays des Gallas, il vient se jeter dans l'océan Indien au sud de Moguedouchou.

1. Partie inférieure de l'appareil génital de la Bloyetia Revoili. A. orifice commun; B. poche vaginale; C. oviducte; D. matrice; E. poche copulatrice; F. fourreau de la rerge; G. flagellum, H. canal déférent. 
en un long flagellum filiforme. Chez les Hyalinies et les Zonites, le flagellum n'existe pas, ou s'il existe, il est réduit à l'état rudimentaire.

En somme, les Bloyeties sont, pour les contrées océano-équatoriales du continent, les représentants de la série des Leucochroa candidissima si abondantes dans la région méditerranéenne.

Bloyetia Revolu, Bourguignat, pl, I, fig. 4-6 (Guillainia Revoili, Bourguignat, Hélixar., p. 17, 1885).

Coq. étroitement perforée, globuleuse, ventrue, conö̈de en dessus, convexe en dessous. Test opaque, assez pesant, crétacé, brillant, grossièrement strié ou plutôt subcostulé avec des malléations sur le dernier tour. Coloration d'un bleu-blanchâtre en dessous, offrant en dessus une large zone roussâtre, interrompue par de nombreuses striations bleuitres, souvent si rapprochées que la zone foncée disparait presque entièrement pour faire place ì une surface presque entièrement bleuâtre. Spire conoïde, assez haute, assez aiguë au sommet, qui est terminé par un apex proéminent lisse, d'un jaune éclatant. Six à sept tours peu convexes à croissance lente, séparés par une suture accentuée. Dernier tour d'une taille médiocre, subcompriméarrondi, obscurément subanguleux à son origine, plus convexe en dessous qu'en dessus et rectiligne à l'endroit de l'insertion. Ouverture oblique, relativement peu ample, échancrée, irrégulièrement semi-ovalaire dans le sens transversal, et intérieurement d'une coloration brillante d'un noir-marron 
très intense. Péristome rectiligne, obtus, non évasé, mais plutôt contracté, intérieurement épaissi. Bord columellaire très faiblement dilaté à sa partie supérieure. Bords marginaux écartés, réunis par une faible callosité d'un noir-marron très brillant. Haut., 15-18; diam., 2I-26 mill.

Bloyeta magxifica, Bourguignat, pl. 1, fig. 7-8, (Guillainia magnifica, Bourguignat, Ilélixar., p. 18, 1885.

Cette Bloyetie, à peu près de même taille et de même coloration que la précédente, se distingue, néanmoins, très nettement de celle-ci, par sa forme plus trapue et plus obèse, par son test plus épais, par son dernier tour moins développé, malgré tout plus gros, moins comprimé à l'origine, enfin d'un aspect plus rond et offrant en dessous une turgescence autour de la perforation, perforation généra, lement un peu peu plus étroite, par son bord péristomal plus épais, plus obtus et sensiblement patulescent à la base, enfin par son ouverture contractée, relativement très étroite et moins ample que celle de la Revoili.

Bloyeta c.enulaxs, Bourguignat, pl. 1, fig. 13 (Guillainia carulans, Bourguignat, Hélixar., p. 19, 1885 ).

Espèce presque aussi convexe en dessus qu'en dessous, à spire moins conoïde et un peu moins élevée; de plus, se distinguant des deux précédentes par une croissance spirale plus accélérée, bien que régulière, par un test un peu moins épais, 
plus fortement martelé au dernier tour qui est moins bien arrondi, par sa belle coloration bleuacée, par son ouverture plus oblique, plus ovalaire dans le sens transversal, entourée par un bord péristomal moins épais et plus délicatement obtus. Les tours, au nombre de sept, sont, en outre, un peu plus convexes et la suture est un peu plus profonde. - Haut., 14-16; diam., 24-25 mill.

Bloyetia compressa, Bourguignat, pl. 1, fig. 10 (Guillainia compressa, Bourguignat, Hélixar., p. 20, 1885).

Espèce déprimée, en cône surbaissé, présentant une surface d'une teinte blanche peu bleuacée et sillonnée, sur le dernier tour, par de robustes stries ressemblant à des côtes; six tours à croissance lente, peu convexes, à suture moins accentuée; dernier tour comprimé, subanguleux, très convexe en dessous; ouverture contractée, relativement petite, peu oblique, transversalement oblongue, avec une angulosité du côté externe et un bord inférieur bien cintré; péristome rectiligne, aigu, fortement épaissi à l'intérieur et très robuste sur le côté columellaire, qui est ì peine dilaté. Haut., 12; diam., 20 mill.

Bloyetia Georgl, Bourguignat, pl. 1, fig. 11 (Guillainia Georgi, Bourguignat, Hélixar., p. 20, 1885).

Espèce ayant un certain air de ressemblance avec le Zingis radiolata (voir p. 22), mais s'en distinguant par une taille plus forte, un dernier 
tour bien moins ample, un ombilic plus ouvert, une croissance spirale plus régulière, bien moins accélérée, etc.

Cette Bloyetie est de toutes celle qui a le test le moins grossièrement strié, le moins calcaire, le moins épais et une coloration pas aussi uniforméw ment bleuacée. On ne remarque, en effet, la coloration bleuâtre que sur le dernier tour, notamment en dessous; la surface supérieure est occupée par une large bande d'un roux-marron peu interrompue, passant au rouge sur les tours médians et finissant par disparaitre vers les supérieurs, qui, comme chez les autres Bloyeties, sont d'une nuance éclatante d'un jaune blanchâtre.

Les tours, au nombre de sept, faiblement convexes, néanmoins séparés par une suture prononcée, s'accroissent très lentement jusqu'au dernier, qui, bien renflé-arrondi, se termine à une ouverture presque verticale, échancrée, hémisphérique, à péristome aigu, droit, mince et réfléchi seulement à la partie supérieure de la columelle, sous la forme d'une petite dilatation triangulaire.

— Haut., 17; diam., 24 mill.

Bloyetia Rochebrexiana, Bourguignat, pl. I, fig. 9 (Guillainia Rochebruniana, Bourguignat, Hélixar., p. 21, 1885).

Espèce se distinguant de la précédente par sa haute forme conique, par son test plus épais, plus blenacé, plus vigoureusement strié, par sa spire plus élancée, conico-tectiforme, par son dernier tour plus rentru, plus globuleux, tout en présen- 
tant, à l'origine, une angulosité qui n'existe pas chez la Georgi, par son ouverture plus exactement hémisphérique, un peu plus haute que large (ce qui est l'inverse chez la précédente) et paraissant plus fortement échancrée par suite de la plus grande convexité de l'avant-dernier tour. - Haut., 20; diam., 23 mill.

Bloyetia Mabilliana, Bourguignat, pl. i, fig. 12 (Guillainia Mabilliana, Bourguignat, Hélixar., p.21, 1885).

Cette espèce diffère des deux qui précèdent par son ouverture moins ample, plus oblique, non aussi exactement hémisphérique, mais plutôt arrondie dans un senslégèrement ovalaire, par son péristome obtus et robuste, épaissi et faiblement patulescent, par un test plıs épais, assez finement strié, d'une nuance blanche-bleuacée uniforme (chez les individus bien conservés), par son dernier tour ventruarrondi et légèrement descendant à l'insertion.

Les tours, au nombre de sept, s'accroissent avec lenteur et régularité; la suture est accentuée et la spire est conoüde sous une forme légèrement arrondie, avec un sommet assez proéminent. - Haut., 13-16; diam., 20-22 mill.

Bloyetia Leroyi, Bourguignat, pl. I, fig. 14.

Espèce fossile plus déprimée que la Mabilliana, à spire non conoïde, mais seulement faiblement convexe. Perforation étroite. Test épais, pesant, assez régulièrement strié. Six à sept tours peu convexes, à croissance serrée, séparés par une 
suture assez accentuée. Dernier tour renfié, bien rond, tout en paraissant néanmoins un peu comprimé, très faiblement subanguleux à l'origine. Ouverture oblique, semi-ovale, dans un sens faiblement descendant. Péristome droit, obtus, épaissi, légèrement patulescent à la base. Bord columellaire robuste. un peu dilaté. - Haut., 15; diam., 22 mill. Dépôts sablonneux de la vallée de l'Ouébi, avec la Mabilliana. 


\section{A RTEMONIDE}

Lorsqu'on est en présence ${ }^{4}$ de la méthode taxonomique qui préside à la distribution des Espèces que les auteurs de l'ancienne École ont préconisée pour les formes du genre Streptaxis, on reste stupéfait. Il y a de tout dans ce genre.

Là où l'on ne croyait reneontrer, ainsi que le nom l'enseigne, que des Espèces caractérisées par un axe dévié, que des coquilles remarquables par leur obliquité spirale, on trouve des formes d'Ilyalinies, de Drépanostomes, d'Hélices, sans ancune trace de déviation, accolées à d'autres formes turbinées parfaitement déviées. Cet assemblage d'Espèces hétéroclites est si étrange, que l'on se demande comment on a pu commettre des réunions aussi antiméthodiques, et comment, parce que ces animaux possèdent une plaque linguale analogue à celle des Glandina et similaire de celle des Testacella, on a pu les classer, en compagnie des Glandina, Streptostylus, Pseudosubulina, Streptostele, Gibbus, etc., dans une seule et même famille, celle des Testacellida.

Pour moi, qui n'ai pas vraisemblablement le jugement aussi sain que l'auteur de ces savantes

1. Nolamment du Munuel antiscientifique de conchyliologie, publié sous le nom de M. P. Fischer, et édité par le libr. Savy (Paris, 1881 à 1887). 
classifications, je n'admettrai jamais que les Glandines, ou les Espèces hyaliniformes ou héliciformes, à axe non dévié, soicnt des Streptaxis de la famille des Testacellidre.

Je considère donc toute la série des Espèces à a.xe non dévié non seulement comme étrangères au genre Streptaxis, mais encore comme n'ayant aucun lien de parenté avec les Testacellidx.

J'admets pour ces Espèces la nouvelle famille des Antemonide, famille qui devra prendre place dans la méthode entre celle des Helixarionida et celle des Helicida.

La famille des Artemonidx comprend toutes les Espèces non turbiniformes à axe non dévié, jadis regardées à tort comme des Streptaxis.

Les Espèces de cette nouvelle famille peuvent, d'après l'ensemble de leurs formes et de leurs signes distinctifs, se répartir en neuf coupes génériques, coupes qui ont cela de remarquable qu'elles ont, chez les Hélicidées, leurs analogues et leurs similaires; toutes ces coupes génériques, à l'exception des Gibbonsia, Tayloria et Colpanostoma, sont américaines. Néanmoins, je crois nécessaire de les passer toutes en revue pour faire connaitre leur ensemble et pour faire comprendre l'importance de chacune d'elles par rapport à celles des Gibbonsia, Tayloria et Colpanostoma, les seules spéciales au continent africain. 


\section{GIBBONSIA 1}

Espèce rappelant comme forme certaines Hyalinies de la série des Incerta, Vasconica, etc.

Spire bien convexe, non déviée. Test mince, brillant, fortement strić (stries lamelliformes flexueusement ondulées). Ombilic profond, ouvert en entonnoir. Ouverture échancrée, hémisphérique. Péristome droit aigu et columelle faiblement dilatée, comme chez les Hyalinies.

Gibbonsia gigas (Streptaxis gigas, Smith, in : Proceed. zool. Soc., Lond., 1881, p. 279, pl. xxxir, fig. 4).

Grande espèce adulte, malgré l'assertion du $\mathrm{D}^{\mathrm{r}}$ Smith, découverte dans les régions intermédiaires du lac Nyassa et de l'Océan Indien.

\section{TAYLORIA?}

Espèces ayant tout à la fois une apparence d'Hyalinie et d'Hélice, ce qui mn'empêche de les assimiler à aucune série de ces deux genres.

Spire convexe-arrondie. Test mince quoique opaque, recouvert par un tissu épidermique fugace, d'un brun-marron. Striations lamelliformes, fluxueuses et ondulées. Ombilic profond, médiocrement ouvert, en entonnoir. Ouverture échancrée, ovalaire dans une direction faiblement descen-

1. En l'honneur du voyageur anglais J. S. Gibbons, de Souhampton.

2. En l'houneur du zelé et savant directeur du Quaterly jourual of conchology, M. John, W. Taylor. 
dante. Péristome relativement épais, bien réfléchi, sauf à la partie supéro-aperturale.

Tayloria ventrosa (Zonites? ventrosa Taylor, Desc. new-spec. east coast Afr. in : Quat. Journ. conch. 1 [ $n^{\circ} 12$ aoùt 1877, p. 251, pl. n, fig. 2]). Petite Espèce de Zanzibar.

Tayloria joubert, Bourguignat, spec. nov . (voir ci-après).

Sous les pierres à Nyantaga, dans l'Outongoué, à 50 ou 60 kilom. avant d'arriver à Oudjiji, sur le lac Tanganika.

\section{HAPPIA 1}

Allmonoceras ${ }^{2}$, Pfeiffer, Versuch anordn. Hélic. in : Malak. Bl. 1855, p. 122.

Coupe générique établie, comme nom de section, pour des Espèces américaines hyaliniformes ressemblant aux formes des groupes de la lucida, septentrionalis, etc., et à celui de la testæ de Sicile.

Coq. ombiliquée (ombilic en entonnoir ou simplement dilaté au dernier tour), déprimée, presque plane en dessus ou peu convexe. Test fragile, trans. parent, hyalinoïde, corné, olivâtre ou jaunacé. Croissance spirale rapide ou serrée. Tours peu nombreux (vitrina) ou nombreux (omalomorpha). Ouverture échancrée, semi-ovalaire ou semi ronde. Péristome simple, aigu.

1. En l'homneur de M. Edouard Happe, voyageur français dans l'Amérique méridionale.

2. Non Ammonoeeras, Lamarck, 1822, nec Ammonoceras, Sowerby, 18'2, gemres créés pour des Céphalopodes fossiles. 
A. Espèce ì croissance spirale rapide.

Happla vitrixa (Helix Vitrina, Wagner, in : Spix Test. Bras. 1827, p. 25 ; et deuxième édit. Chemnitz [Helix], pl. Lxxxiv, fig. 14-16). - Brésil.

Happia Cuzcaxa (Helix Cuzcana, Philippi, in : Malak. Bl., 1869, p. 37; Pfeiffer, Novit. p. 476, 1869, pl. cıl, fig. 22-24; - Helix Baezensis, Hidalgo, 1869). - Pérou.

Happia flona (Helix flora, Pfeiffer, in : Zeitschr. Malak. 1850 , p. 68 , et $2^{\circ}$ édit. Chemnitz [Helix] $n^{0}$ 796, pl. cxxril, fig. 1-2). - Colombie.

Happia Morobambensis (Helix Moyobambensis, Moricand, in : Rev. et mag. zool. 1858, p. 450, pl. xııı, fig. 3). - Pérous.

B. Espèce à croissance spirale servée.

Happia omalomorpha (Helix omalomorpha, d'Orbigny, Synops, p. 5, 1837, et Voy. amér. Moll., p. 259, pl.xxvir, fig. 1-3. - Iyalinia omalomorpha, Albers, 1860). - Bolivie.

Happia onbicula (Helix orbicula, d'Orbigmy, Syn. p. 6., 1837, et Voy amér. Moll. p. 270, pl. xxvir, fig. 16-19). - Bolivie.

Happia trochiliones (Helix trochilioides, d'Orbigmy, Syn., p. 6, et Voy. Amér. Moll., p. 251, pl.xxru, fig. 12-15). - Bolivie et Pérou.

\section{SCOLODONTA}

Doring, in : Boletin. acad. cienc. Cordoba. 1875, p. 4.38 . 
Espèce ressemblant à des Hyalinies de la série des Crystallines d'Europe.

Coq. de petite taille, largement ombiliquée, déprimée, tantòt presque plane en dessus (Semperi $i$, ou faiblement convexe, tantôt subconoïde (Paraguayana); croissance spirale régulière, généralement à tours serrés; test délicat, brillant, cristallin, strié; ouverture hémisphérique; péristome ordinairement simple, aigu, parfois bordé.

Scolodonta semperi (Streptaxis [Scolodonta] Semperi, Docring [Loc. sup. cit.], p. 439). République Argentiue.

Scolodonta ammoxoceras (Helix ammonoceras, Pfeiffer, in : Proc. zool. Soc. Lond. 1854, p. 54, et Monogr. Hel. viv. rv, 1859, p. 104). - NouvelleGrenade.

Scolodoxta argextixa (Hyalina Argentina, Strobel, Malac. Arg. p. 9, pl. I, fig. 1, 1874). - République Argentine.

Scolononta Thomasi (Helix Thomasi, Pfeiffer, in: Proc. zool. Soc. Lond., 1854, p. 54). - Nouvelle Grenade.

Scolodonta spirorbis(IIelix spirorbis, Deshayes, in : Ferussac. Hlist. Moll., I, p. 83). - Brésil.

Scolodonta cualicophata (Helix chalicophila, d'Orbigny, Syn., p. 6, 1837, et Voy. Amér. Moll., p. 251, pl. xxru, fig. 8-11). - Bolivie.

Scomonoxta simpula (Helix Skiaphila, dorbi- 
gny, Syn., p. 5, 1837, et Voy. Amér. Moll., p. 249, pl. xxvir, fig. 4-7). - Bolivie.

Scolodonta mutata (Helix mutata, Gould, Expéd. Shells, p. 19, 1846). - Brésil.

Scolodonta Bounobena (Belix Bounobæna, d'Orbigny, Syn., p. 7, et Voy. Amér. Moll., p. 253, pl. xxvirl, fig. 17-20). - Brésil.

Scolodonta myephila (Helix hylephila, d'Orbigny. Syn., p. 7, 1837, et Voy.A mér. Moll., p. 253, pl. xxvul, fig. 13-16). - Bolivie.

Scolodoxta acitephila (Helix achtephila, d' $\mathrm{Or}$ bigny, Syn., p. 7, 1837, et Voy. Amér. Moll., pl. xxvir, fig. 9-12). - Pérou.

Scolodonta paraguayua (Helix Paraguayuna, Pfeiffer, Symb. Helic., II, 1842, p. 93. - Helix elevata ${ }^{1}$, d'Orbigny, Syn., p. 6, 1837, et Voy. Amér. Moll., p 254, pl. xxvin, fig. 5-8).-Paraguay. Cette Espèce a la forme d'un Conulus d'Europe

\section{DREPANOSTOMELLA}

Espèce hyaliniforme ressemblant, en dessus, à une Hyalinia cellaria et rappelant, par les caractères de l'ombilic, de l'ouverture et du mode de projection du labre externe apertural, les Diepanostoma de Lombardie; de là le nom de Drepanostomella.

Coq. orbiculaire, largement ombiliquée en en-

1. Non Helix elevata, Say, 1818, Espèce de l'Améríue du Nord. 
tonnoir, à test fragile, transparent, brillant, presque lisse. Spire concave, enroulée sur un plan presque horizontal. Tours peu nombreux, embrassants en dessus. Ouverture ovalaire, caractérisée par un bord externe se projetant en avant et domnant lieu, par suite de sa grande projection, à un simulacre de sinus supéro-apertural.

Drepanostomella amonitiformis (Helix ammonitiformis, d'Orbigny, Syn.,p. 5, 1837, etVoy. Amér. Moll., p. 243, pl. xxvi, fig. 10-13.-Ammonoceras ammonitiformis, Doring, 1875). - Brésil et Bolivie.

\section{COLPANOSTOMA}

Espèce ressemblant comme forme générale à l'Helix fruticum, mais en différant essentiellement par une échancrure à la partie supérieure de la paroi externe.

Coq. ouvertement ombiliquée, ventrue, à spire bien convexe. Test transparent, opalin, très brillant, sillonné par de fortes radiations lamelliformes flexueuses. Ouverture échancrée, presque ronde, entourée par un bord péristomal nettement réfléchi, sauf à la partie supérieure du bord externe, à l'endroit de l'échancrure.

Colpaxostona leroyt, Bourguignat, sp. nov. (Voir ci-après.)

Endroits boisés, à une altitude de 2000 mètres, sur le N'gourou, massif de montagnes au nord de

1. Etymologie : ouverture échancréc dans le haut. 
la haute vallée du Vouami, entre l'Oukamba et l'Ousaghara.

\section{A RTEMON 1}

(Beck, Ind. Moll., p. 48, 1838, et Pfeiffer, Vers. anordn. Helic., in : Malak., BI., 1855, p. 172).

Genre établi pour des espèces ressemblant à des Hélices de la série des Strigella.

Test transparent, d'une teinte opaline. Striations lamelliformes régulières et accentuées. Spire convexe. Croissance spirale normale. Ouverture échancrée, subovalaire et subarrondie. Péristome brièvement réfléchi ou non réfléehi. Ombilic cireulaire bien ouvert.

Artemox candidus, Beck, Ind. Moll., p. 38, 1838 (Helix Spixiana, Pfeiffer, Symb. Helic., I, 1841, p. 40; Helix perspectiva, Wagner, 1827; Hélix candida, Moricand, 1841; Solarium eandidum, Spix, 1827). - Brésil.

Artemon intemaneres (Streptaxis intermedius,

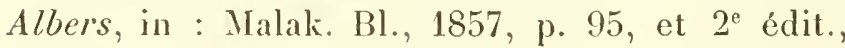
Chemnitz (Helix), p. 15, pl. cur , fig. 26-28 [Sous le nom de Streptaxis Wagneri, var. Pfeiffer]). Brésil.

Artenon ubenfonmis (Streptaxis uberiformis, Pfeiffer, in: Proc. zool. Soc. Lond. 1848, p. 108,

1. Les auteurs domnent pour étymologie le mot $\alpha \rho \tau \varepsilon u(\theta) v$ (voile d'artimon, ou poulie d'une moufle). Cette étymologie est fausse. Beck a dû tirer ce nom générique du mot opteuro (frais), parce que le test des Espèces est brillant et d'une teinte fraîche. 
et Hidalgo, in : Journ. Conch.. 1875, pl, ru, fig. 8 . - Brésil.

Artemon Wagrem (Helix Wagneri, Pfeiffer, Symb. Helic., I, 184L, p. 40, et $2^{e}$ édit., Chemnitz, [Helix], pl.xix, fig. 4-6; Helix coffreana, Moricand, 1841). - Brésil.

Antemon costulosus (Ilelix costulosa, Pfeiffer, in : Proc. zool. Soc. Lond., 1852, p. 136, et $2^{\mathrm{e}}$ édit., Chemnitz [Helix], pl. cxul, fig. 29-31). - Iles Salomon.

Les Espèces apertus, alveus, Paivanus, cypsele, Rollandi, conoideus et le turbinatus (Morelet) d'Angola, mentionnées dans le Nomenclator Heliceorum de Pfeiffer, ne peuvent faire partie de ce genre.

\section{DISCARTEMON}

(Pfeiffer, Vers. anordn. Helic, in: Malak. B1., 1855, p. 175).

Espèces déprimées, héliciformes, ressemblant par l'ensemble de leur's caractères aux Hélices du groupe des Diodonta (Muhlferldt) et Camerani (Lessona) d'Europe.

Coq. largement ombiliquée, discoüde, à spire peu convexe (sauf chez le Crossei, qui est subconoïde); test assez résistant, plus ou moins opaque ou subtransparent, offrant des parties variqueuses aux arrêts d'aceroissement; coloration d'un ton cristallin blanchàtre on verdâtre; ouverture dentée; péristome bordé, plus ou moins 
réfléchi, épaissi intérieurement à son contour inférieur.

La spire chez les Discartemon n'offre aucune déviation streptaxiforme; si en dessous, chez le discus, le dernier tour semble un tant soit peu dévié, cela tient à ce que l'ombilic, chez cette Espèce, en prenant au dernier tour une expansion ellipsoüde, donne à ce tour un aspect excentrique.

Discantemon disces (Streptaxis discus, Pfeiffer, in : Proc. zool. Soc., Lond., 1851, p. 252, et $2^{\mathrm{e}}$ édit., Chemnitz [Helix], pl. cxtr, fig. 15-17). - Brésil.

Discantenox chrptonox (Helix [Streplaxis] cryptodon. Moricamel, in : Journ. Conch., 1851, p. 370, pl. x, fig. 2). - Brésil.

Duscamenox Crosser (Streptaxis Crossei, Pfeiffer, in: Journ. (ronch. 1867, p. 43, pl. I, fig. 1). Brésil.

\section{ALCIDIA}

Espèces ressemblant à des Hélices de la série de la conica, ou plutòt à des Sagda du groupe de la Sagela alligaus de la Jamaïque.

Coq. obluse, turbince, à spire élerie, arrondie on forme de dóme; lest bien strié on costulé, transparent, d'une teinte blanche-jaunacée ou olivàtre; tours nombreux très serrés; dernier tour subanguleux ou subarrondi; ouverture petite, hémisphérique; ombilic profond très étroit alveus ou

1. Prénom du célébre zoologiste et paléontologiste. Alcide d'Orbign'ly. 
très ouvert en entonnoir et laissant voir l'enroulement interne (cypsele).

Alcidia cripsene (Helix cypsele, Pfeiffer, in : Zeitschr. Malak., 1849, p. 78, et $2^{\text {e }}$ édit., Chemnitz. [Helix], p. 384, pl. xı*, fig. 8-10). - Brésil.

Alcidia apenta (Streptaxis apertus [pars, exclud. var.], Martens, in : Malak. Bl., 1868, p. 180, et Pfeiffer, Monogr. Hel. viv., VII. 1876, p. 49 [pars]). - Brésil.

Alcodi alveus Streptaxis alveus, Philippi, Abbild. Helix, pl. vt, fig. 14, et $2^{\mathrm{e}}$ édit., Chemnitz [Helix], pl. ci, fig. 33-35). - Brésil.

Alcond Paraxa Streptaxis Paivana, Pfeiffer, in: Journ. Conch. 1867, p. 43, pl. 1, fig. 2). - Brésil.

Tel est l'ensemble des genres et des Espèces qui composent la famille des Artemonidx. On remarquera que toutes les formes spécifiques, saul quatre, sont spéciales à l'Amérique du Sud.

Je passe aux descriptions des deux Artémonidées africaines inćdites que j’ai à faire connaître.

TAYLORLA JOLBERTI, spec. nov. (pl. it, fig. 6-9)

Testa aperte umbilicala (umbilicus relative medioeris, nihilominus sat apertus, profundus ac subpervius!, depresso-convexa, solidula, opacula, nitidula, epidermide fugaci fusco-castaneo induta et eleganter striato-sublamellosa (lamella oblique undulate ac flexuosa ad basin ultimi evanescentes); - spira sal produclo-convexa, obeso-rolundata, 
ad summum perobtusa; - anfractibus 6 arctespiratis, convexiusculis, sutura angusta profundaque separatis; - ultimo rotundato, nihilominus leviter superne convexo-subtectiformi; - apertura parum obliqua, lunata, semiovata in directionem descendentem; - peristomate prope insertionem recto, externe et ad basin incrassato ac reflexo; margine columellari valido, dilatato-reflexo; - callo palatali tenui, late expanso; - alt., 10; diam., 7 ; alt. ap., 4 ; lat., $41 / 4$ millim.

Chez cette Taylorie, le maximum de la convexité du dernier tour s'accuse un peu au-dessous de la partie périphériale.

Cette Espèce se distingue de la Tayloria ventrosa de Zanzibar, par sa taille plus forte, par sa croissance spirale plus lente, par ses tours plus nombreux, par son ouverture plus ovalaire-descendante, par son péristome plus épais, plus réfléchi, et par son bord columellaire plus robuste et plus dilaté,

COLPANOSTONA JEROYI, spec. nov. (pl. I, fig. 1-3)

Testa profunde umbilicata, ventrosa, solidula, subopacula, nihilominus subtranslucida, nitidissima, uniformiter crystallino-opalina, inferne polita, superne eleganter striato-radiatula (strixe flexuosæ, regulares, circa suturam productre, validiores, quasi costulatæ ad basin ultimi omnino evanescentes); - spira convexa, rotundata ; anfractibus 6 (superiores exigui, levigati) convexiusculis, sat lente crescentibus, sutura impressa 
separatis; ultimo rotundato; - apertura obliqua, lunata, subrotundata, superne ad insertionem rimata, intus albo-margaritacea; - peristomate undique (preter ad partem superam) incrassato ac bene reflexo; margine externo superne sinuatolunato ae subter antrorsum curvato-provecto et dein recte retrocedente; callo palatali insconspicuo; alt., 20; diam., 25; alt. ap., 11; lat., 13 millim.

Cette singulière Espèce, des sommets du mont N'gourou, remarquable par la forte échancrure qui caractérise le sommet de son ouverture, ressemble beancoup comme forme à l'Helix fruticum, dont elle se distingue, dı reste, par sa coloration, le brillant de son test. le mode de ses striations, etc.

D'après un échantillon conservé dans l'alcool, le manteau de cet animal, vu par transparence, m'a paru jaune et moucheté de nombreuses taches noires. 


\title{
I I I
}

\author{
BUL1MES DU GROUPE DE
}

\section{L'A BYSSINICUS}

Les Bulimes de ce groupe, remarquables par leur test sillonné de stries lamelleuses, fines, serrées, régulières, qui leur donnent un air de parenté indéniable, appartiennent aux trois séries suivantes:

A. Espèces de forme ventrue-ovoïde ou oblongue, à spire plus ou moins atténuée, saus être ni comoìde ni pyramidale, et à péristome toujours patulescent du côté externe.

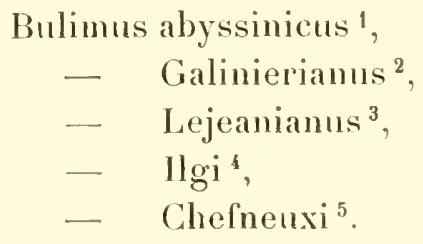

1. L. Pfeiffer, in : Zeitschr. f. Malak, p. 157, 1845, et Monogr. hel. viv., II, p. 110, 18ł8, et Jickeli, Moll. n. ost. Afr., 1874, p. 102, pl. v, fig. $2 \mathrm{~A}$ et C seulement. - Espèce de l'Abyssinie et du Choa.

2. Bourguignat, Malac. Abyss., p. 56 et 113, fig. 60, 1883. - De l'Abyssinie.

3. Bourgnignat, Mal. Abyss., p. 57 et 114, fig. 61, 1883. 1)e l'Abyssinie.

i. Soleillet, in : Bourgnignat, Moll. Choa, p. 11, 1885. - Du Clioa.

5. Snleillet, in : Bourgniguat, Moll. Choa, p 12, 1885. - Dn Clunit. 
B. Espèces de forme allongée, à spire acuminèe, à péristome également patuleseent du côté externe.

\section{Bulimus Hemprichi !,}

- Lonrdeli, spec. nov.

C. Espèces de forme oblongue-allongée ${ }^{2}$ ou inférieurement ventrue, aree une spire acuminee ou conoide et un péristome droit non patulescent du côté exterue.

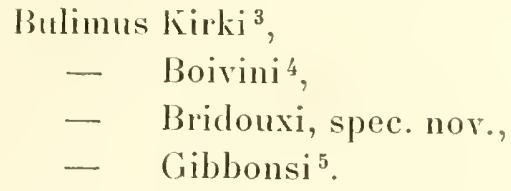

Chez les Espèces de la première série, la perforation est relativement très ouverte; chez celles des denx autres, elle est bien plus réduite.

BLLIMUS LOLRDELI, spec, nov. (pl. In, fig. 2-3)

Testa perforata (perforatio profunda, angustaque), elongato-pyramidali, inferne relative ventrosa,

1. Jickeli [Buliminus], Moll. n. ost. Afr., 1874, p. 106, p]. v, fig. 3. - De l'Abyssinie et dı Choa.

2. Comme le Bul. Boivini.

3. Dohrn [Buliminus], in Proc. zool. Soc. Lond., 1865, p. 232. - Bulimas Kirki, Smith, in : Proc. zool. Soc. Lond., 1881, p. 282, pl. xxx11, fig. 9. - De Mozambique, de l'Ousaghara, de l'Ougogo, etc.

亿. Bonrguignat, in : Grandidier, Moll. Ousagh., in : Bull. Soe, malac. Fr., IV, 1887, p. 187 (Glandina Boivini, Morelet, 1860). - Cả et là des côtes de l'Océan à la région du Tanganikit.

5. Taylor, in : Quat. journ. conch., 1er nor. 1877, p. 280, pl. ın, fig. 1. - Cette Espèce a été retronvée par le commandiut Bloyet aux envioms de Kondoa. 
solidiuscula, subopacula, nitida, cornea, eleganter subcostulato-striata (strix conferte regulares, validx, costulas simulantes); - spira elongata, pyramidata, ad summum obtusa (apex obtusus, sublevigatus ac incolor); - anfractibus 7-8 tumidorotundatis, lente arcteque crescentibus, sutura profunda separalis; - ultimo rotundato, dimidiam altitudinis non requante, ad insertionem recto, iufra cirea perforationem vix turgidulo; - apertura vix obliqua, valde lunata, semiovato-rotundata; peristomate recto, leviter incrassatulo, undique patulescente; columella robusta, recta, dilatatoreflexa. - Nt., 18; diam., 10; alt. ap., 7; lat., $51 / 2$ millim.

Cette Espèce, dédiće au Rér. P. Lourdel, a été recueillie dans l'Ousaghara, aux environs de Kondoa.

Ce Bulime, qui ne peut être rapproché que du Bulimus llemprichi (Jickeli), se distingue de celuici par sa forme plus allongée-pyramidale, plus ventrue au dernier tour, par sa spire un peu plus longue, par son sommet plus obtus, par ses tours plus gonflés, plus rouds, par conséquent par une suture plus profonde, par son ouverture fortement échancrée, semi-ovale, presque semi-ronde, très ample a la base, bien dans l'axe de la coquille et non un peu inclinée à droite comme celle de l'Hemprichi, par sa columelle rectiligne, enfin par ses bords marginaux très écartés, non convergents, comme ceux de l'Hemprichi. 
BULIMUS BRIDOUXI, spec. nor. (pl. II, fig. 4-ä).

Testa anguste perforata (perforatio nihilominus sat aperta), ventroso-subconoidali, soliclula, subopacula, parum nitente, cornea, valide costulatostriata (stric conferta ac regulares); - spira conoidali, mediocriter producta, ad summum subacutiuscula (apex minutus); - anfractibus 7 convexis, lente crescentibus, sulura subprofunda separatis; - ultimo tumido, subrotundato-convexo, dimidiam altitudinis non aequante, ad insertionem recto, inferne circa perforationem turgidulo; apertura parum obliqua, lunata; irregulariter ovata, superne angulata, inferne leviter angustala; - peristomate recto, acuto, intus leviter subincrassatulo; columella valida, brevi, recta ac dilatato-reflexa; - Mlt., 19; diam., 11 ; alt. ap. 9 ; lat., 7 millim.

Espèce abondante sous les pierres, le long des broussailles, entre Kondoa et Mpouapoua, dans l'Ousaghara.

Ce Bulime, auquel j'attribue le nom de Mgr Bridoux, vicaire apostolique du Tanganika, diflère du Bul. Kirlici (Smith), la seule espèce avec laquelle il peut être assimilé, par sa forme plus ventrue, plus courte, plus trapue, par sa spire moins allongée, plus conoüde, par sa perforation un peu moins ouverte, par son dernier tour plus gros, ventru et plus rond, égalant presque la moitié de la haı- 


$$
-54-
$$

teur', et moins renflé autour de la perforation, enfin, par ses stries coslulées plus accentuées et un peu moins serrées.

1. Che le kirli, le dernier tour est loin d'atteindre la moilié de la hauleur. 


\section{RACHIS'}

Ce genre, tel qu'il a été établi par le Dr Albers (Die Heliceen, p. 182, 1850), est certainement un des genres le plus mal définis qui existent.

L'auteur lui attribue comme caractère une coquille perforée, turriculée-conique ou ovale; une spire acuminée, plus longue que la hautenr du dernier tour; cinq à six tours, dont le dernier sonvent subanguleux à la périphérie; une ouverture ovale; un péristome simple aigu, avec un bord columellaire dilaté et rélléchi.

A l'appui de sa diagnose, le $D^{r}$ Albers eite huit Espèces, réparties en Espèces à test mince, diaphane, et en Espèces à test mince et solide; savoir:

\section{Test mince et diaphane.}

\section{Bulimus pallens, Jonas, - Guineensis, Jonas, - Ferussaci, Dunker.}

1. Doit-on écrire Rachis, ou Rhachis comme l'enseigne le docteur Martens. Raehis est tiré du mot p̉x $x /^{10}$, épine dorsale, par extension axe columellaire. Le docteur Marlens (in : $2^{\circ}$ édit. d'Albers, p. 231 ) écrit Rhachis, en donnant ì ce mot la signification de Rückgrut spindel, ce qui signifierait axe rétrocédant. Quoi qu'il en soit de ce nom générique, mal digéré, il doit s'orthographier Rachis et non Rhachis. 
Test opaque et solide.

\section{Bulimus Mozambicensis, Pfeiffer,}

- Benguelensis, Pfeiffer,

- albatus, Férussac,

- Socotrensis, "

- connivens, Pfeiffer.

Sur ces huit Espèces, trois seulement possèdent les caractères rachisidiens, les Bulimus pallens, Mozambicensis, et Benguelensis.

Les cinq autres appartiennent a des groupes génériques différents :

Le Guineensis doit être un Opeas 1 ,

Le Ferussaci est un Rachisellus ${ }^{2}$,

L'albalus est un Limicolaria,

Le socotrensis, un Ovella ${ }^{3}$,

Le connicens, un Bulimus.

Dans la seconde édition des Heliceen du $\mathrm{D}^{\mathrm{r}} \mathrm{AI}-$ bers, édition publiée par les soins du $\mathrm{D}^{\mathrm{r}}$ Martens,

1. Ce genre, établi également par le docteur Albers (Hélic.,

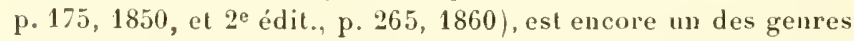
le plus mal défini, geure qui aurait besoin d'ètre bien déliuilé, parce que dans cette coupe générique, les auteurs, chacun a leur fantaisie, $y$ ont compris des formes très disparates les unes des aulres.

2. Voir ci-après.

3. Genre élabli par Pfeiffer (Nomencl. Hel. viv., p. 284, 1878). pour le Bulimus socotrensis. - Les Ovella sont les Socotrensis, hadibuensis, Bal/ouri, Gollons rensis, ligris, zebrinus, longiformis, semicastaneus de l'ile de Socolora. - Ce genre avait été primitivement établi par Nevill (Hand. list. Moll. ind. museum Calcuttit, I, p. 131, 1878), sous le nom antimétho- 
en 1860, les caractères de la diagnose première restent à peu près les mêmes, seulement le Dr Martens donne pour type générique le Bulimus punctatus, d'Anton ${ }^{1}$, qui, d'après la figure de Kuster (2 $2^{\mathrm{e}}$ édit., Chemn., pl. Lxil, fig. 22-24), parait par sa petite taille, sa forme oblongue, atténuée à la base, sa perforation ombilicale plus ouverte, etc., appartenir a la série des Rachisellus, série qui a pour lype l'Espèce si commune sur les côtes africaines du Zanguébar et du Mozambique, le variolosus; enfin l'on trouve mentionnées près d'une vingtaine de formes spécifiques, dont la plupart ne possèdent pas les caractères voulus par la diagnose.

Le type Rachis, ainsi que l'a indiqué Albers en 1850, est le Bulimus pallens de Jonas, et nul autre.

Cette Espèce type, telle qu'elle est figurée (pl. I, fig. 3) dans Philippi, est une coquille conoïde, a spire acuminée, à croissance spirale régulière, subtectiforme, avec un dernier tour subanguleux, n'égalant pas la moitié de la hauteur, caractérisé, en outre, par un bord péristomal simple, aigu, du còté externe, et par un bord columellaire dilaté seulement au sommet sous la forme d'une petite réflexion trianguliforme.

Ces caractères cadrent bien avec ceux reconnus par le Dr Albers.

dique d'Achalinelloides. (Voir au sujet des Espèces de Socolora, l'exeellent Iravail du lieutenant-colonel Godwin-Austen [Socotr. shells, in : Proc. zool. Soc. Lond., 1881.])

1. Bulimus punctatus, Anton, Verz., p. 42,1839 , et Pfeiffer, Monogr. Hel., II, p. 212, 1848, et Kuster, Bul. (2e édit., Chemnitz), pl. Lxu, (ig. 22-2i). - Espèce de l'Indoustan. 
Les Espèces que je considère comme appartenant véritablement à cette coupe générique sont :

1. Raculs rallexs (Bulimus pallens, Jomas, in: Plilippi, Abbild., I [3 livr., 1843], p.53, pl. ı, fig. 3 [nom Bul. pallens de Pfeiffer, qui est l'Espèce suivante]).

De Guincée.

2. Ricus Cineroxi (Bulimus Cameroni, Bourg., Moll. Egypte, Abyss., Zanzibar, etc., p. 4, 1879; - Buliminus pallens [non Jomas], Pfeiffer, Novitales, 1876, pl. cxxxvu, fig. 11-12; - Rhachis Finsli, Dorhll, mss. teste Pfeiffer).

Cette Espèce, que l'on rencontre à Zanzibar, et ‘́a et là dans les vallées du Vouami et du Kyngani, diffère du type figuré par Philippi, par sa coquille, d'une taille supérieure, ornée de une à deux bandes marron, par son dernier tour moins anguleux et notamment parson ouverture plus arrondie du côté externe et plus portée en dehors. Chez le pallens de Jonas, le coté externo-apertural, peu convexe, presque recto-descendant. n'offre pas cette dilatation arrondie de la base externe qui caractérise l'ouverture du Cameroni [pallens de Pleiffer].

Je posside des échantillons parfaitement adultes et bien semblables au type de Jonas, de même que j’ai également en ma possession des exemplaires bien similaires à la coquille figurée dans les $\mathrm{No}$ rilates. En somme, sous le nom de pallens, Pfeiffer a décrit, en les croyant plus adultes, des individus d'une lispèce diflérente. 
3. Racins Rochebunxinus (Pachnodus Rochebrunianus, Bourg. Malac. Abyss., p. 79, pl. iv, fig. 81,1883$)$.

De l'Alsyssinie.

4. Rucins Vescol (Bulimus Vesconis, Morelet, sér. conch., II, 1860, p. 65, pl. v, fig. 1. - Pachnodus Vescoi, Bourg., Malac. Abys., p. 79, 1883).

Je Madagascar.

5. Ruculs Sprker (Bulimus Spekei, Bourg., Moll. Egypte, Abyss. Zanzibar, ete., p. 4, 1879, et Pachnodus Spekei, Bourg., Mal. Abyss., p. 79, 1883.)

Zanzibar et Zanguebar.

6. Ricurs subcoxicus (Limicolaria subconica, llartens, Conch. Mittheil. Nov. conch. II $\left[1^{0 s}\right.$ v et vi 1885], p. 188, pl. xxxir, fig. 3-4).

Loango (Guinće méridionale).

7. Ricus Bruvxs, Martens. (Buliminus [Rh] Braunsii, Llartens, Conch. Zanzibar, in : Nachrichsblatt. 1869 , p. 150, et Pfeiffer, Novitates, n"722, pl. cxin1, fig. 11-12, 1870).

Lanzibar.

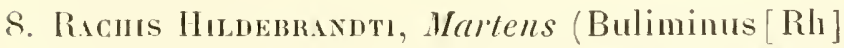
Braunsii, var. Hildebrandti, Martens, Moll. Ilildebr. in : Monatsbericht. akad. Wiss. Berlin, 1878, p. 294, pl. 11, fig. 1-2).

Còtes des Bénadirs, dans la vallée de l'Ouébi et aux environs de Guélidi, a 4 à 5 jours de marehe a l'oceident de Moguedouchou. 
9. Rachis rhodotran, Martens (Buliminus [Rh.] rhodhotinia, Marlens, Moll. Decken, 1869, p. 59, pl. 11, fig. 2, et Moll. Hildehr., 1878, p. 292 , pl. 11, fig. 7).

Zanguébar et Zanzibar.

10. Raculs picturutus (Bulimuspicturatus, Morelet, Coq. nouv. Afr. mérid., 1889, p. 3, pl. 1, fig.4). Zanguébar.

11. Racins stictus, Marlens (Bulimus [Rh.] stictus, Marlens, Moll. Peters Mossamb. in : Malak. Bl., 1859, p. 211, pl. 11, fig. 6).

Mozambique.

12. Rachis Gatexatus, Marlens (Bulimus [Rh.] catenalus, Martens [Loc. su]). cit.], p. 212, pl.11, fig. 7) ${ }^{\mathrm{s}}$.

Mozambique.

13. Racins Blovet1, Bourguignat, in sched. 1886.

Cette Espèce est très bien représentée dans Smith (Shells. tang. in : Proc. zool. soc. Lond, 1881, p. 281, pl. xxxu, lig. 7. ${ }^{\mathrm{A}}$.) , sous le nom erroné de Bulimus [Rh.] Braunsii de Martens, Bulime avec lequel cette coquille n'a pas le moindre rapport. Le Rachis Bloyeli est, en effet, parmi les Rachis, l'Espèce la moins anguleuse au dernier tour, la forme dont la spire est le moins pyramidale, les tours le moins tectiformes, et l'ouverture le plus ample.

1. Les deux autres Espèces que le docteur Martens publie dans le mème mémoire, sous les noms de Bulimus melanacme et punctalus, en les eonsidérant comine des Rachis, ne me paraissent pas appartenir à ce genre. 
Cette Espice est commune en Mozambique et dans le Zanguébar. Je la possède des environs de Kondoa (Ousaghara), ou les échantillous sont bien semblables à ceux représentés par Smith, sous le nom erroné de Braunsi. On la rencontre également sur les monts N'gourou, au nord de l'Ousaghara, jusqu'à une altitude de $2000 \mathrm{~m}$.

J'arrête à cette Espèce la liste des Rachis ${ }^{1}$, liste que l'on pourrait allonger si on le voulait, mais les Especes que je viens de citer sont suffisantes pour que l'on puisse juger, par elles, du véritable caraclère du genre lachis. Je passe a la description de trois formes inćdites.

RACIIS JOLBERTI, spec. nov. (pl. 11, fig. 10-12).

Testa oblique rimata (rima perangusta, fere omnino tecta), pyramidali, crassula, opacula, substriatula, candida cum zonulis 2 fusco-filiformibus quarum una ad peripheriam, altera inferior prope rimam; - spira tectiforme-conica ; apice acuto; anfractibus 7 vix subconvexiusculis aut potius tectiformibus, regulariter crescentibus, sutura lineari separatis; - ultimo majore, dimidiam altitudinis non attingente, ad initium quasi subangulato, ad insertionem leviter descendente; - apertura perobliqua, mediocriter lumata, subovato-rotundata; - peristomate recto, acuto, intus incrassato; mar-

1. Dans le Nomenclator heliccorum de Pfeiffer (p. 28' et 285), l'on constate une liste de trente-neuf Espèces considérées comme Rachis; je dois avouer que la pluparl de ces Espèces n'appartiennent pas a ce groupe générique. 
gine columellari valde retrocedente, roseo, in lamellam triangularem late reflexo; - alt., 17; diam. 9 ; alt. ap., $71 / 2$; lat., 5 millim.

Cette Espèce, qui ressemble comme forme à l'Hildebrandti, est remarquable par l'extrême obliquité de son ouverture et par la forte rétrogradation de son axe columellaire, qui paraît comme renversé en arrière. Chez le Jouberti, la dilatation trianguliforme du sommet de la columelle est relativement plus développée que celle que l'on observe chez les autres Rachis.

Mont Kidété, près le lac Ongombo, entre Kondoa et Mpouapoua (Ousaghara).

RACHIS PACHISTOMA, spec. nov. (pl. III, fig. 8-9)

Testa obtecte perforata, pyramidali; sat crassa ac opaca, striatula, ommino candida; - spira producto-conica; apice acuto; - anfraetibus 7 convexiusculis, regulariter crescentibus, sutura impressa separatis; ultimo majore, subangulato (angulus prope aperturam evaneseens), convexo-rotundato, sat tumido, dimidiam altitudinis non attingente, ad insertionem reeto; - apertura obliqua, lunata, semiorata; - peristomate crasso, obtuso, intus labiato ae undique patulo; margine eolumellari robusto, superne medioeriter dilatato ac nihilominus perforationem omnino tegente; - alt., 20; dian.. 11; alt. op., 9; lat., 6 millim.

Environs de Guélidi, à l'onest de lloguedouchou (Madagoxo). 
C'est le seul Rachis, a ma comnaissance, qui ait un test aussi opaque et un bord columellaire épanoui, aussi épais. La perforation ombilicale est complètement obstruéc par le renversement de la réllexion columellaire.

RACHIS ELONGATULUS, spec. nox. (pl. m, fig. 6-7).

Testa punctiforme perforata (perforatio perangusta, fere omnino tecta), turrito-acuminata, solidula, subopacula, striatula, candida ad summum violacea et zonulis tribusviolaceo-vinosis obscurecircumcincta; - spira elongata, acuminata; apice acuto;--anfractibus 8 convexiusculis, regulariter crescentibus, sulura lineari, inter ultimos impressa, separatis; ultimo sat turgido, convexo, dimidiam altitudinis non attingente, ad insertionem recto;apertura obliqua, lumata, semiovata, intus subviolacea cum zonulis violaceo-vinosis bene apparentibus; - peristomate recto, acuto; margine columellari tenui, violaceo-vinoso, superne mediocriter dilatato ac nihilominus perforationem fere omnino tegrente;-alt., 23 ; diam., 9 ; alt. ap., 9 ; lat., 6 mill.

Cette Espèce, la plus allongée des Rachis, remarquable par le développement de sa spire, vit dans la vallée de l'Ouébi, à cinq ou six jouts de marcheà l'occident de Moguedouchou (Magadoxo). 


\section{V \\ PACHNODUS}

On trouve dans la seconde édition (1860) des Heliceen d'Albers (p. 230), sous le nom de Pachnodus, une coupe générique ainsi définie :

Coq. perforée ou très rarement imperforée, de forme ovale-conique, à test mince, strié ou décussé. Cinq à six tours, le dernier grand, ventru, égalant la spire qui est courte, conique, à sommet aigu. Ouverture ample, ovalaire ou ovale-oblongue. Péristome simple, fragile, à bord columellaire dilaté et réfléchi au sommet.

En somme, d'après ces caractères, l'on voit que les Pachnodus ne sont que des Rachis à spire courte, an dernier tour très ample égalant ou dépassant mème la moitié de la hauteur.

C'est par erreur que le Dr Martens désigne comme type de cette coupe générique le Bulimus velutinus des îles Seychelles ', c'est plutôt, saus aucun doute, le Bulimus spadiceus, que cet auteur a voulu indiquer. L'erreur est possible, du reste, parce que ces Bulimes sont tous les deux représentés sur la mème planche (pl. Lxi) de Kuster? l'un, le selutinus, aux figures 11 et 12 , l'autre, le spadiceus, ì relles 13 et 14 .

1. Bulimus relutinus, Pfeiffer, Symb. hist. Hel., I, 1842, p 42, et Monogr. Hel., II, 1848, p. 192 (Helix strigillosa, Férussac, mss., et Bulimus strigillosus, Dufo, mss, leste P'feiffer).

2. Gatt. Bulimus (2rédit., Chemnitz). 
Le Bulimus selutiuus ne convient en ancune facon. D'après les figures 11 et 12, e'est une forme bulimoüde, ovale-ventrue, à spire obtuse, tandis que le spadiceus (fig. 13-14) est une Espèce conö̈de à spire brièvement acuminée au dernier tour égalant la moitić de la hauteur. Le spadiceus est done un Pachnodus.

Les principales Espèces de cette coupe générique sont :

Pacmodus spanceus, Menke, in : Philippi, Ablild., II, p. 123 (1846), Bul., pl. v, fig. 3.

Forêt sur le bord du fleuve Umlaa (Natal).

Pachoones xitalexsis (Bulimus natalensis, Krauss, Moll. Sudafric, p. 78, pl. r, fig. 1, 1848). Forèts du Natal.

PACHNoDts LEROYi, spec. nov. (pl. III, fig. 4-3)

Testa vix rimata (rima fere omnino tecta), ventroso-conica, fragili, nitida, subpellucida, argute striatula, albicante et passim pallide griseo-marmorata, ad summum nigrescente, in ultimo superne nigro-punctata (puncti irregulariter sparsi) ac zomulis duabus (una ad peripheriam, altera inferior) fusco-circumcincta; - spira breviter conica; anfractibus 6 parum subeonvexiusculis, regulariter usque ad ultimum crescentibus, sutura subimpressula separatis; - ultimo maximo, amplo, tumido, ventroso, rotundato, dimidiam altitudinis leviter superante, ad insertionem recto; - apertura parum obliqua, lunata, ovata, superne sat angulata, intus 
cum punctis ac zonulis apparentibus;-peristomate recto, fragili, acuto; margine columellari tenui, superne triangulari forma late dilatato ac super rimam reflexo; - alt., 12; diam., 9; alt. ap., 7; lat., 4 1/2 millim.

Celte belle Espèce, qui ne peut être confondue avec le spadicens el le natalensis, vit à une altitude de $2000 \mathrm{~m}$., sur le mont N'gourou (Ousaghara), où elle a été découverte par le Rér. P. Leroy.

PAChNodus SEsahorum, spec. nov. (pl. iI, fig. 2-3)

Pachnodus Sesamorum, Ancey, mss.

Testa anguste rimata, ventrosa, conoidea, solidiuscula, subopacula, nitida, subtiliter striatula, subcarneolo-albescente, ad summum nigrescente et in ultimo punctis atris parum numerosis ornata ac ad peripheriam atro-mizonata; - spira brevi, conica; - aufractibus 5-6 convexiusculis, regulariter usque ad ultimum crescentibus, sutura impressa separatis; ultimo magno, tumido-rotundato, dimidiam altitudinis superante, ad insertionem leviter descendente; - apertura obliqua, parum lunata, subrotundato-ovata, intus cum punctis et zonula apparentibus; - peristomate recto, acuto, intus sublabiato; margine columellari brevi, superne in triangulari forma late dilatato; marginihus approximalis ac convergentibus; - alt., 10; diam., $71 / 2$; alt. ap., 6 ; lat., 4 millim.

Espèce abondante dans les sacs de Sésame provenant du Mozambique. 
Ce Pachnodus diflère du précédent, par sa taille moindre, par son test plus solide, moins transparent, d'une coloration différente, par sa spire moins régulièrement conique par suite des tours qui sont plus gonflés, par son dernier tour descendant à l'insertion, par son ouverture plus arrondie, par son péristome intérieurement bordé, dont les bords marginaux sont rapprochés et convergents. 


\section{I}

\section{RAGHISELLUS}

Les Espèces de cette nouvelle coupe générique ont été jusqu'ici confondues avec les Rachis et les Pachnodus.

Les Rachisellus sont des coquilles qui, au lieu d'être inférieurement ventrues avec une spire allongée-conoïde plus ou moins tectiforme et plus longue que le dernier tour (Rachis), ou, an lieu d'offrir une spire conoïde plus courte (Páchnodus), sont caractérisées par un test oblong-allongé, pourvu d'un dernier tour, non gros ni ventru, mais atténué à la base, ce qui lui donne un aspect fusiforme.

Chez les Rachisellus, la perforation est ouverte et profonde, la dilatation columellaire au lieu d'être supérieure et trianguliforme, descend en pointe jusqu'à la base de l'axe, enfin, le dernier tour est subanguleux autour de la perforation.

Les Espèces de ce genre ont une apparence si différente de celle des Rachis et des Pachnodus que je suis à me demander comment on a pu si longtemps les laisser confondues avec les formes de ces deux genres. Elles ressemblent en effet à des Limicolaires minuscules, et, parmi les Limicolaires, je ne vois gुuère que celles des pays Comalis ${ }^{1}$

1. Telles que les Limicolaria Revoili, Gilbertæ, Maunoiriana, Perrieriana, Rochebruni, Armandi, Leontina, Milne-Edwardsi et Rabaudi (voir Mission Reroil, Moll. du pays Comalis, 1882). 
qui peuvent avoir quelques affinités d'aspect avec elles.

Les Rachiselles semblent des Espèces spéciales aux contrées litlorales du sud de l'Afrique. On les rencontre en abondance sur les plantes et les arbrisseaux, depuis la côte d'Ajan jusqu'à celles de la Guinée.

Ricinselues idextatus (Bulimus punctalus, Inton, Verz. Conch., p. 42, 1839; - Kuster, Bul. [20 édit., Chemnitz], n" 322, pl. rxu, fig. 22-24; Deshayes, Moll. Ferussac, II, p. S6, pl. Cuvir, fig. $7 \cdot 8)$.

Cetle Espèce (type du genre Rachisellus), primilivement découverte dans l'lude, a été relrouvée abondamment dans l'ile de Zanzibar et sur toute la côteafricaine depuis Moguedouchou (Magadoxo) jusqu'à Nozambique.

Riacinseluus Burtor, Bollguignat, sp. nov.

Cetle Espéce diffère de la précédente par sa perforation plus ouverte, laissant apercevoir $u 1$ trou profond el entouré par une angulosité relalivement très prononcée; par une spire plus pyramidale, à tours plus convexes, par conséquent à suture plus accentuće, mais notamment par son dernier tour plus renflé-arrondi a lorigine et ne devenant comvexe-oblong que vers l'ouverture moins oblique, qui est entourée par un bord péristomal aigu, non patulescent à la base.

Au point de vue de la forme, le Burtoi, quoique a pen pros de mème laille, parait moins allongé et 
n'a pas l'aspect fusiforme du punctatus, bien que son dernier tour soit également atténué inférieurement.

Sur les plantes marines, le long des côtes entre Brava el Monbas.

Rachiselles vaholoses (Bulimus variolosus, Morelet, Sér. Conch., II, p. 66, pl. v, fig. 2, 1860; - Pfeiffer, Monogr. Helic., VI, p. 139, 1868 . Rachis variolosa, Pfeiffer, Nomencl. Hel. viv., p. 285, 1878).

Plage de Moguedouchou (Magadoxo) au pied des plantes arénicoles.

Racuiselues Ferussaca (Bulimus Ferussaci, Dunlier, in : Zeitschr. 1. Malak., 1845, p.164; - Pfeiffer, Monogr. Hel. viv., II, 1848, p. 212; - Menlie, Moll. Guin., p. 6, pl. I, fig. 35-36, 1853; - Rachis Ferussaci, Pfeiffer, Nomencl. Hel. viv., p. 285, 1878.

Coteaux arides près de Loanda (Angola).

Racinsellus Ledoulxi, Bonirguignat, sp. nov. (pl. v, fig. 10-11).

Coq. profondément perforée, oblongrue-allongée, fusiforme, fortement atténuée inférieurement, à test transparent, fragile, très finement striolé, d'une nuance jaune-paille interrompue par des flammules transverses blanchâtres d'un ton assez opaque, et ceinte à la périphérie du dernier tour par une zonule filiforme brune. Spire acuminée. Sept tours convexes, à croissance lente et régulicre, séparés par me suture assez profonde. Dernier 
tour convexe-oblong, anguleux autour de la perforation, et n'atteignant pas la moitié de la hauteur. Ouverture oblique, oblongue, anguleuse au sommet, étroite à la base. Péristome mince, droit, aigu. Bord columellaire délicat, réfléchi en une dilatation triangulaire allongée en pointe jusqu'à la base de l'axe. - Haut., 14; diam., 7; haut. ouv., 6; larg. 4 1/4 millim.

Sur les plantes marines à Zanzibar et sur la côte africaine à Sadani, Pangani, Tanga et Monbas. 


\section{VII}

\section{AGHATINA}

J'ai à faire connaitre plusieurs formes nouvelles; mais je crois utile, avant de donner leurs caractères, d'émettre unon opinion sur la distribution des nombreuses Achatines, pour montrer la position relative que doivent occuper ces formes nouvelles dans la classification des Espéces de ce genre.

Le genre Achatina, établi par Lamarek', a pour type l'Achatina sariegata (Bulla achatina de Linneus), Espèce très bien représentée sur lesplanches du grand ouvrage de Férıssac (pl. cxxxı, fig. 1.3 et $\operatorname{cxxxl}^{\mathrm{A}}$ fig. 1 [senlement] et $\left.\mathrm{cxxxi}^{\mathrm{B}}\right)$.

Ce genre a pour caractère un bord péristomal droit, jamais réfléchi du côté externe; un manque complet de perforation; une columelle constamment mue et tris lisse, enfin toujours nettement tronquée à la base.

Primitivement, on avait rangé dans cette coupe générique des multitudes d'Espèces hétérogènes de l'ancien et du nonveau Monde, Espèces qui, pen à peu, ont été éliminées, et finalement ce genre est resté un ğenre essentiellement africain. Tel qu'il est resté, ce genre a subi encore des modifications; Albers, en 1850, a créé à ses dépens ses Psendachatina, Shuttleworth, en 1856, ses Perideris, coupes génériques excellentes qui, si

1. Prodr., p. 75, 1799, et Syst. Anim., p. 90, 1801, et Auim. sans rert., VI, 2e partie, 1822, p, 126. 
elles n'avaient pas été élablies, s'imposeraient.

Malgré ces deux réductions et colles opérées par Incey dans les Bulletins de la Société malacologique de France, je crois qu’il est encore néeessaire de distraire des Achatines les trois coupes suivantes:

$1^{\circ}$ Tripachatina (ou Achatine perforée) pour l'I chalina lignoniana, du Gabon. (Morelet, in : Journ. conch. 1874, p. 172. Voir à ce sujet Ancey, Nouv. cont. malac. in : Bull. Soc. malac. Fr., 1I, 1885, p. 137, au paragraphe relatif aux Espèces africaines).

Cette Espece, type dn Tripachatina (Bourg.), est rematquable par une troncature oblique et peu accusée et par une perforation ombilicale ouverte, circonscrite par une forte angulosilé formant une crête obtuse.

Les vraies Achatines n'ont ancme trace de perforation ${ }^{1}$. leur columelle est toujours constamment nue et très lisse, comme l'a dit Lamarck.

$2^{\circ}$ Parachatina (Bourg.) Cette coupe générique est établie pour les:

Achutixi thousoxi, Smith, in: Proc. zool. Soc. Lond., 1881, p. 283, pl. xxxin, fig. 12.

Contrées entre la côte et le lac Nyassa.

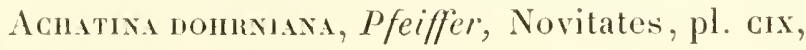
fig. 1, 1870.

Angola.

1. Pas plus que les Pseudachatina d'Albers (Helic., p. 192, 1850 . créé pour l'Achatina Downesii de Gray, genre dans le-quel quelyues auteurs ont compris, at tort, la Viguoniana. 
Achatima Welwitscin, Morelet, Moll. Welw., p. 66, pl. v, fig. $2,1868$.

Angola et Benguela.

Etc.....

Ces Espèces sont caractérisées par leur manque de troncature columellaire, troncature remplacéc par une éminence tuberculeuse.

$3^{\circ}$ Serpæa (Bourg.). Coupe générique ('rée pour des petites Espèces gloluleuses, d'un aspect tont particulier (Voir ci-après page 85)

Malgré ces éliminations, ce genre comprend encore de nombreuses Espèces; elles sont toutes alficaines; elles penvent se répartir en cinq séries.

A. SÉrie ré La varlegata. Coq. de grande taille, de forme oblongue plus ou moins allongee et ventrue. Test médiocrement épais. Onverture ample, oblongue, souvent allongée, fortement anguleuse au sommet, ordinarement rétrécie à la base, enfin égalant ou dépassant le plus souvent la moitié de la hauteur.

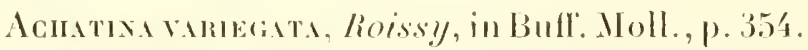
(Buccinum variegatum exoticum, Fab. Columna; Bulla achatina, Limneus, 1758, ete... Achatina perdrix, Lamarcli, 1822).

Région du Gahon. Espèce bien représentée dans Férussar pl. cxxxt, lig. [-3).

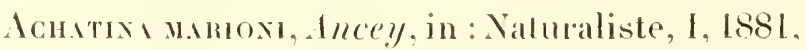
p. 414 .

Contrées entre Brava el le Nanza Oukéréwé. 
Acmatima immaculata, Lamarcl, Anim. s. vert., VI, $2^{\text {e }}$ partie, 1822, p. 129.

Cap Delagoa, au sud du Mozambique. Espèce bien figurée dans Férussac, pl. cxxvı.

Acinativi Liyardi, Pfeiffer, in : Malak. Bl., 1858, p. 238.

Oiba, sur la côte orientale. Bonnes figures dans les Novitates, pl. xu, fig. 1-2.

Acilatixa monetaria, Morelet, Moll. Welw., p. 63, pl. viı, fig. 1-2.

Benguela.

Achativa Schwemfuntm, Martens, in : Malak. Bl. 1873, p. 40; Pfeiffer, Novitates, pl. cxxxu, fig. 1-2; Jickeli, Moll. n. ost. Afr', p. 150, pl. vi, fig. 1, 1874.

Régions du hati Nil.

Achatixa Paxtuera, Desheryes, an. s. vert., VIII, 1838, p. 309. (Helix [Cochlitoma] panthera, Férussac, 1821.)

Zanzibar, Zanguebar et Madagascar. Espèce bien figurée dans Férussac (pl. cxxvı. fig. 1-2).

Acintixa zaxzibarica, Bourguignat, Moll. Egyple, Abyss., etc.., p. 5, 1879.

Zanzibar.

Achatixa Lhotelleri, Bourguignat (Loc. sup. cit.), p. 7, 1879.

Zanzibar.

Achatixa Hamllew, Petit, in : Journ. conch., 1859, p. 384, pl. xı11, fig. 3; Smith, in : Proc, zool. Soc. Lond.. 1881, p. 282, pl. xxxı1, fig. 10. 
Centre de l'Afrique et régions occidentales.

Achatra Cravexi, Smith, in : Proc. zool. Soc. Lond., 1881, p. 283, pl. xxxm1, fig. 11.

Régions entre la còte orientale et le Tanganika.

Achatisa fulica (non Philippi), Martens, Moll. Decken, 1869, p. 58, pl. 11, tig. 1 (Helix [cochlitoma] fulica, Fépussac, 182i, et Helix Borbonica, Férussac).

Madagascar. - L'Achatine, publiée, sous le nom de fulica, par Philippi (abbild. III, Ach. pl. In, fig. 3) n'est pas la fulica de Martens; sa columelle n'est pas nettement tronquée.

Achatixa zebra, Lamarcli, An. s. vert., VI, $2^{e}$ partie, 1822, p. 128; Kuster, (2 ${ }^{\mathrm{e}}$ édit. Chemnitz), pl. II, fig. 3 (Bulla zebra de Chemnitz).

Madagascar et régions du Cap et de Natal. Cette Espèce est très bien représentée dans Férussac (pl. cxxxun, fig. 1-2 seulement); la petite Achatine intermédiaire (fig. 3), considérée comme jeune, est adulte, et est la Serpxa obesa (voir ci-après).

Acmatixa ranicosa, Pfeiffer, Novitates, $n^{0} 65 \mathrm{l}$, pl. crr, fig. 1-2. (Non Achatina varicosa, Pfeiffer, in : Malak. Bl., 1861, p. 73, pl. II, fig. 7-8, et Monogr. Hel. viv., VI, 1868, p. 215, regardée comme une forme non adulte. Cette Espèce appartient au grence Serpea).

Enon, Capp de Bonne-Espérance.

Achatina Romata, Dunlier, in : Zeitschr. f. malak., 1852, p. 127, et Pfeiffer, Monogr. Hel. viv., III, 
1853, p. 483, et Novitates, pl. xxvu bonnes figures).

Zanzibar.

Achatixa rurpuea, Lamarch, Anim. s. vert., VI, $2^{\mathrm{e}}$ partie, 1822, 1). 128; et Poties et Michand, Moll. Gal. Douai, I, 1838, p. 130, pl. xu, fig. 3-4(bonnes),

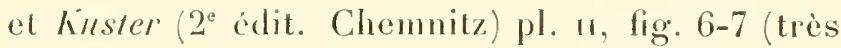
médiocres ). - Bulla purpurea de Chemnitz.)

Còte de Malaguette (Afrique occidentale).

Achatma ponpmostoma, Shutterrorth, in : Bern. Mittheil., 1852, p. 201, et Pfeiffer, Monogr. Ifel. viv., III, 1853, p. 483.

Côte occidentale.

Acmatran monostoma, Plilippi, Abbild. III, p. 29, Ach., pl. II, fig. 2, et Kuster (2 ${ }^{\mathrm{e}}$ édit. Chemnitz), pl. xill, fig. 3 .

Còte occidentale.

Acintixa rostom, Pfeiffer, in : Proc. zool. Soc. Lond., 1852, et Monogrr. Hel. viv., III, 1853, p. 485, et Kuster (2e édit. Chemnit\%), pl. xum, fig. 3.

Fernando-Po.

Achatixa gletrosi, Pfeiffer, in : Proc.zool. Soc. Lond., 1852, et Monogrr. Hel. vir., III, 1853, p. 485, et ( $2^{\circ}$ édil., Chemnitz) pl. xur, fig. 1.

Còte occidentale.

Acmotina Baxumana, Morelet, Moll. Welw. p. 67, pl. vi, fig. 1, 1868.

Benguela et Angola.

Achatra Parvaxa, Morelet, Moll. Weluv., p. 65, pl. vi, fig. 3, 1868.

Congo. 
Acilatina perfecta, Morelel, Moll. Welw., p. 70, pl. iv, fig. 2, 1868.

Côte occidentale.

Achativa suturalis, Philippi, Abbild. III, Ach., p. 29, 1849, pI. 11, fig. 1 (non Achatina suturalis de Pfeiffer, Monog. Hel. viv., II, 1848, p. 284, qui est une Streptostyla de l'ìle de Cuba).

Côte occidentale.

Achativa Petersi, Martens, in Malak. Bl., 1859, p. 214, et Pfeiffer, Monogr. Hel. viv., VI, 1868, p. 213, et Novilates, pl. xcix, fig. 13-14.

Mozambique.

Acinativa balteata, Reeve, Conch. iconogr., $n^{0} 7$, pl. II, et Pfeiffer, Monogr. Ilel. viv., III, 1853, p. 487.

Sénégambie. - Espèce bien représentée dans Férussac (pl. cxxxı, fig. 3-4).

Acilatina fulva, Beck, ind. Moll., p. 76, 1839, et Deshayes, An. s. vert., VIII, 1838, p. 309, et Kuster ( $2^{\mathrm{e}}$ édit. Chemnitz), pl. xı, fig. 3-4, et Férussac, pl. cxxiv, fig. 1-2.

Côte occidentale.

Achatina sinistronsa, Pfeiffer, Monogr. Hel viv., II, 1848, p. 248 (Bulla achatina sinistrorsa, Chemnitz, 1786 ; Bulimus bicarinatus, Bruguière; Achalina bicarinata, Lamarck, etc... etc...). Espèce bien représentée dans Férussac (pl. cxxvıi); Kuster en a donné une représentation moins exacte (pl. xv, fig. 3-4) dans la seconde édition de Chemnitz.

Iles du Prince, de San Thomé, etc... 
J'arrète à cette Espèce la liste des formes de cette série, parce que je serais entrainé trop loin si je voulais toutes les citer.

B. SÉHe de ha Letourneuxi. Coq. très allongée, relativement peu ventrue pour la hauteur. Test épais, solide et assez pesant. Ouverture ordinairement médiocre, plutôt ovale qu'oblongue, parfois subarrondie et inférieure en hauteur à la moitié de la longueur totale.

Achatixa Letourneuxi, Bourguignat, Moll. Égypte, Abyss., etc., p. 8, 1879.

Zanzibar, Zanguébar et còte des Bénadirs.

Achatixa Muge-Enwadi, Révoil, in : Bull. Soc. malace. Fr., II, 1885, p. 98, pl. v, fig. 1.

Ousaghara et còte des Bénadirs.

Achatina neticulata, Pfeiffer, in : Proc. zool. Soc. Lond., 1845, p. 74, et Monogr. Itel. viv., II, 1848, p. 252.

Zanzibar et Zanguébar. Espèce très bien représentée dans Férussac (pl. cxxix), et dans la deuxième édition de Chemnitz (pl. xxvı, fig. 1).

Achatina Plaxti, Pfeiffer, in : Proc. zool. Soc. Lond., 1860, et Novitates, n² 256, pl. xum, fig. 1-2. Natal.

Achatixa Blovet, Bourguignat, spec. nov. (Voir (ci-après, page 82).

Ousaghara.

C. SÉme de l'dictespirata. Coq. de petite taille. 
Spire acuminće, à sommet obuus. Croissance spirale lente. Columelle courte.

Achatixa arctespirata, Bourguignat, spec. nov. (Voir ci-après, page 83.)

Contrées au sud du Tanganika.

Achatixa spegularis, Morelet, Moll. Welw., p. 74, pl. Ir, fig. 4, 1868.

Còte occidentale (Benguela et Angola). C'est la plus petite des Achatines.

D. SÉrie de LA Randabeli. Coq. de moyenne taille, a test mince, délicat, de forme oblongue glandinoüde.

Achatixi Raxdabel, Bourguiguat, spec. nov. (Voir ci-apres, page 84.)

Ounyanyembé, entre le Tanganika e 'Ousaghara.

Achativa semisculpta, Pfeiffer, in: Proc. zool. Soc. Lond., 1845, p. 74, et Dunlier, Moll. Guinće, p. 7, pl. 1, fig. $42-43,1853$.

Côte de Guinće.

Achatina ustulata, Lamarek, Anim.s. vert., VI, $2^{\circ}$ partie, 1822, p. 130. (Helix [cochlitoma] ustulata, Férussac, 1821.)

Cap de Bonne-Espérance. Espèce bien figurée dans Férussac (pl. cxxr, fig. 1-2).

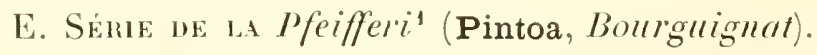
Coq. ressemblant à des Limicolaires.

1. Quelques auteurs ont regarde à tort la Pfeifferi comme un Homorus. Les vais Homorus n'apparliennent pas à la famille des Achatinida. 
Spire obtuse allongée. Croissance spirale lente. Ouverture ovale ou semi-arrondie, relativement petite, n'égalant jamais la moitié de la hauteur. Columelle courte.

Achatima Pfeiffeni, Dunker, Moll. Guin., p. 7 , pl. I, fig. 39-40, 1853, et Philippi, Abbild. Aeh., p. 213, pl. I, fig. 2, 1847.

Loanda (côte occidentale).

Achatina semidecussata, Menle, in : Philippi, Abbild. Ach., p. 213, pl. 1, fig. 1, 1847.

Natal.

Achatina Capelloi, Furtado, in : Jomm. conch., 1886, pl. vi, fig. 2.

Mozambique.

Achatixa zebrola, Morelet, Moll. Welw., p. 72 , pl. III, fig. 1, 1868.

Côte occidentale.

Achativa Polychnod, Morelet, in : Journ, conch., 1866, p. 158, et Moll. Welw., p. 72, pl. III, fig. 5, 1868.

Côte occidentale.

Acintixa colubmin, Morelet, Moll. Welw., p. 70, pl. IV, fig. 1, 1868.

Côte occidentale.

Ces Espèces, que je viens de mentionner, sont suffisantes pour faire comprendre la distribution des formes achatinifoïdes. 
ACHATINA BLOYETI, spec. nov.

Testa elongata, nihilominus relative tumida, opaca, nitidula, ad supremos levigata, in creteris striata ac in ultimo grosse striato-lamellosa, albescente cum flammulis rufo-castaneis ; - spira elongato-acuminata, ad summum acutiuscula; - anfractibus 7-8 convexis, regulariter crescentibus, sutura impressa separatis; - penultimo ad dextram notabiliter turgido; - ultimo convexo-oblongo, ad aperturam rotundato, dimidiam altitudinis non attingente, superne lente descendente; - apertura parum obliqua, lumata, irregulariter ovata, externe rotundata; - columella recta, abrupte truncata, ad basin non descendente; - peristomate recto, acuto; callo palatali albido; - alt., 105; diam., 53; alt. ap., 51 ; lat., 31 millim.

Cette Espèce, dédiée au capitaine Bloyet, qui l'a recueillie aux environs de Kondoa (Ousaghara), est remarquable par sa spire acuminée, pourvue de tours relativement très convexes, et notamment par son avant-dernier, ainsi que par son dernier tour, fortement renflés, arrondis du côté droit, caractère qui donne à la suture, entre ces deux tours, une concavité très prononcée, concavité qui fait ressortir la rotondité de ces tours.

J'ajouterai que chez cette Achatine, très distincte de celles de sa série, l'axe columellaire recto-descendant se troncature bien avant la base de l'onverture. 
ACHATINA ARGTESPIRATA, spec, nov. (pl, v, fig, i)

Testa obeso-tumida, in medio ventrosa, opacula, nitidula, argute striata, uniformiter pallide lutescente cum flammulis rufo-costaneis leviter fulguratis (sub epidermide albo-eretacea); - spira acuminato-pyramidali, ad summum obtusa (apex validus, obtusus, quasi submamillatus); - anfractibus 8 convexiuseulis, arctespiratis, sutura sat profunda separatis; - ultimo magno, dimidiam altitudinis superante, oblongo-convexo, inferne attenuato; - apertura parum obliqua, elongata, sat angustata, superne perangulata, inferne relative aretata ; - columella brevi, reeta, abrupte truncata; - peristomate recto, acuto; callo tenui, diaphano; - alt., 57 ; diam., 32 ; alt. ap., 31 , lat., 16 millim.

Chez cette Espèce, les flammules du dernier tour ne sont pas fulgurantes jusqu'a la base, mais prennent brusquement, à partir de la périphérie, une direction spirescente descendante d'arrière en avant. C'est la seule Achatine sur laquelle j’ai observé c'e mode de flammules.

L'arctespirata est remarquable par sa spire, qui bien ru'avec un gros sommet obtus, est pyramidale-acuminée, tout en offrant une apparence trapue-écourtée. La croissance spirale est fort lente, le dernier tour est notablement atténué inférieurement, ee qui le fait paraitre plus renflé à sa partie médiane.

Cette Espece est très abondante dans toute la 
région méridionale du Tanganika, où on la rencontre très rarement en bon ćlat, par suite des incendies allumés par les naturels du pays, incendies qui, en détruisant son tissu épidermique, rendent son test d'un blane mat uniforme.

ACHATINA RANDABELI, spec. nov. (pI. v, fig. 6)

Testa oblonga, mediocriter ventrosa exacte glandiniformi, sat tenui, subpellucida, nitidula, argute decussata (anfractus supremi ac ad partem inferiorem ultimi lavigati), castaneo-subolivacea, cum flammulis rubris leviter fulguratis et interruptis irregulariter sparsis; - spira obtuse acuminata, ad summum obtusa (apex rotundatus); - anfractibus 8 convexiusculis, sat lente crescentibus, sutura impressa separatis; - ultimo oblongo, tumidulo, dimidiam altitudinis superante; - apertura parum obliqua, lunata, irregulariter oblongo-elongata, ad summum acute angulata; peristomate recto, acuto; - columella recta, abrupte truncata; - callo inconspicuo ; - alt., 55; diam., 28 ; alt. ap., 31 ; lat., 15 millim.

Cette Espèce, très distincte des Achatines de sa série, a été recueillie aux alentours de Tabora, dans l'Ounyanyembé. Elle est dédiée au Rév. P. missionnaire Randabel du Tanganika. 


\section{VIII}

\section{SERPAA}

Les Serpex se composent d'une série de formes aussi dissemblables des Achatines que le sont celle des Perideris, ou bien celles des Pseudachatina, Campylaxis, Eutaxis, Callistoplepa, etc., que l'on a eu raison de distraire du genre Achatina.

Les Serpara se distinguent par une forme écourtée, ovö̈de-ventrue ou presque sphérique, à test mince et transparent. Ces Espèces sont anx Achatina ce que les Burtoa sont aux Limicolaria. Ce groupe très naturel mérite à tous les points de vue d'être séparé sous un nom générique spécial.

Les Serpea connues sont:

Serpea hontensie (Achatina Hortensia, Morelet; Moll. Welw., 1868, p. 74, pl. iv, fig. 3). Du Benguela.

Semper daminensis (Achatina Dammarensis, Pfeiffer, in : Malak. B1., 1870, p. 31, et Novitates, $\mathbf{n}^{\circ}$ 674, pl. cix, fig. 3-4). - Dammara, au sud d'Angola.

Senper Kixon (Achatina Knori, Jonas, in : Wiegm. Archiv., 1839, I, p. 345, et Pfeiffer, Monogr. Hel. viv., II, 1848, p. 250, et III, 1853, p. 485, et in : Chemnitz (2 édit.), Bul., pl.xurn, fig.11-12. Achatina prunum, Reeve, Conch. iconogr., n" 13, pl. iv). - De l'Afrique occidentale (Habitat inconnu). 
Serpea valucosa (Achatina varicosa, Pfeiffer, in : Malak. Bl., VIII, 1861, p. 79, pl. 11, fig. 7-8, et Monogr. Hel. viv., VI, 1868, p. 215 [non, Achatina varicosa, Pfeiffer, novitates, III, p. 490, $n^{\circ} 651$, pl. cru, fig. 1-2]).

Cap de Bonne-Espérance. Je m'étonne comment Pfeiffer, avec son coup d'oil et son regard exercé, a pu se tromper au point de considérer sa raricosa de 1861 comme un jeune de sa varicosa des Novitates.

Serpea obesa (Achatina obesa, Pfeiffer, in : Malak. Bl., 1854, p. 224, et Monogr. Hel. viv., IV, 1859, p. 600).

Afrique occidentale. - Belle espèce parfaitement représentée (pl. cxxxı, fig. 3 [fig. intermédiaire]) dans l'ouvrage de Férussac, où elle est faussement considérée comme une jeune Achatina zebra, ce qui est absurde.

SERPEA PINTOI, Bourg., spec. nov. (pl. iv, fig. 4)

Testa ovoideo-ovata, ventrosa, fragili, pellucida, striatula (anfr. supremis exceptis), ac in ultimis eleganter lineolis spiralibus decussata, uniformiter pallide castanea, flammulis intentioribus interruptisque ac subfulguratis obscure ornata, ad summum albescente; - spira sat brevi, obtuse acuminata; - anfractibus 6 convexis, regulariter usque ad ultimum crescentibus, sutura relative profunda separatis; - ultimo magno, dimidiam valde superante, ventroso, ad insertionem lente descendente; - apertura parum obliqua, oblongo-ovata, superne 


$$
-87-
$$

angulata, inferne bene rotundata; - peristomate recto, acuto; margine columellari subcurvato, sat brevi, ad basin non attingente ac abrupte truncato; - callo palatali tenuissimo; - alt., 49; diam., 33; alt. ap., 32; lat., 19 millim.

Cette belle Espèce, très distincte de celles qui précèdent, comme l'on peut s'en convaincre par la comparaison des figures, provient des environs de l'Ougerengere, vallée du Kyngani, dans l'Oukani. Elle est dédiće aul célèbre voyageur portugais, Serpa Pinto. 


\section{IX}

\section{BURTOA}

Cette nouvelle coupe générique, que j'établis en l'honneur d'un explorateur français des contrées équatoriales de l'Afrique, se compose d'Espèces classées jusqu'à présent parmi les Bulimes et les Limicolaires.

Ces Espèces, d'un aspect tout particulier, sont ventrues, de forme ovoüde, à spire courte, ordimairement obtuse, pourvues d'un test assez mince, décussé, sur la partie supérieure des derniers tours, par des sillons transversalx et spiraux, et reconvert d'un tissu épidermique fugace d'un ton marron accentué, sillonné, de plus, chez la plupart, de longues flammules transverses de nuance plus claire, s'alternant avec d'autres très foncées; enfin, offrant, sur le pourtour apertural et sur la columelle, une coloration rose-violacée, passant parfois an blenàtre.

Chez ces Espèces, l'axe columellaire fortement dilaté sous la forme d'une réflexion triangulaire très allongée, qui recouvre plus ou moins la perforation, descend tantôt rectilignement jusqu'à la base de l'axe, tantòt s'arrète un peu au-dessus en donnant lieu alors à un semblaut de retrait subachatiniforme.

Les Burtoies paraissent spéciales atux rastes régions qui s'étendent depuis les bassins des 
grands Nyanzas du cours supérieur du Nil jusqu’a la terre de Natal.

Les huit Burtoies, qui me sont actuellement connues, peuvent être rangées en deux séries, d'après leur axe columellaire.

A. Axe columellaire pourvu d'une dilatation descendant en pointe jusqu'à la base de l'ouverture :

Burtoa nilotica,

- Bourguignati;

- Raymondi,

- Bridouxiana.

B. Axe columellaire ne descendant pas tout à fait jusqu'à la base, offrant à sa terminaison, soit une légère éminence lamelleuse, soit un semblant de retrait subachatiniforme:

Burtoa sebasmia,

- Kraussi,

- Pethericki,

- Lavigeriana,

Plusieurs auteurs avaient considéré les Espèces chez lesquelles on remarque un semblable axe columellitire comme des formes non adultes. J'ai eu, entre les mains, assez d'exemplaires de ces Espèces ainsi caractérisées pour reconnaitre qu'elles étaient parvenues à leur taille el à leur grosseur.

Burton xilotica (Limicolaria nilotica, Pfeiffer, Novilates, n" 676, pl. cx, fig. 1, seulement).

de ne mentionne pas dans la synonymie de celte Espèce le Bulimus niloticus de P'eifier (in : Proceed. 
zool. Soc. Lond., 1861, p. 24, et in : Malak. Bl., VIIJ, 1862, p. 14, et Monogr. Hel. viv., VI, 1868, p. 86), des sourees du Nil Blane; ni la Limicolaria nilotica de Dohrn (in : Proceed. zool. Soe. Lond., 1864, p. 116'), de l'Ouganda et du Karagoua; ni l'Achatina (Limic.) nilotica de Martens (in: Malak. Bl., 1865 , p. $196 ; 1866$, p. 94; 1870, p. 30; 1873, p. 38), des sources du Nil Blanc; ni enfin, l'itchatina nilotica de Jiekeli (Moll. n. ost. Afr., p.151, 1874), ete..., parce que je crois que sous ces appellations se cachent plusieurs formes différentes, d'autant plus que les descriptions de Pfeiffer ne concordent pas exactement entre elles. Ce qui vient à l'appui du doute que j'expose, e'est que, sur la planche cx des Novitates, où ont étédonnées les premières figurations de cette Espèce, l'on remarque, sous le nom de nilotica, trois figures qui incontestablement ne peuvent se rapporter à une seule et même Espèce. La figure 3, qui a le même nombre de tours que la figure 1, n'est point le jeune du numéro 1, ainsi, du reste, que le démontre, an surplus, la facture des tours supérieurs. De mème également, le numéro 2, malheureusement représenté de dos, par sa forme oblongue-bulimoïde et la patulescence inférieure de son bord columellaire ${ }^{2}$, ne peut être assimilé aux deux autres.

En somme, sur cette planche, il y a trois formes distinctes: fig. 1, la niloticrque jeconsidère comme le type du grenre Bartoa; fig. 3, une forme spéciale

1. Sans description.

2. Cette forme doil appartenir au grenre Burtopsis. 
adulte, à laquelle j'attribue le nom de Pethericki; enfin, fig. 2, une forme indéterminable, qui doit être un Burtopsis.

Le type nilotica, tel qu'il est représenté pl. cx, fig. I (sculement), est une grosse Espèce (h., 112; d., 74; h. ouv., 74; 1., 49 millim.) ovoüde, très ventrue, a spire courte, obtusément acuminée, terminée par un sommet émoussé, obtus, caractérisée, en outre, par six tours convexes, dont le dernier énorme, ventru-arrondi, égale près des $2 / 3$ de la hauteur, par une grande ouverture très dilatée inférieurement, notamment du côté externe où le contour est bien arrondi; par un péristome droit, faiblement obtus, non patulescent; par un bord columellaire réfléchi en une dilatation triangulaire-allongée descendant rectilignement en pointe jusqu'ì la base de l'axe, sans donner lieu à aucune sinuosité.

Cette forme typique pourrait bien être celle que Pfeiffer signale des sources du Nil Blanc, ou des contrées où l'on croyait, à cette époque, que se trouvaient ces fameuses sources actuellement encore incounues.

Burtos Boungugnati (Limicolaria Bourguignati ${ }^{1}$, Grandidier, Moll. cent. Afr., in : Bull. Soc. Malac. Fr., II, 1885, p. 157, pl. vu, fig. 1).

Cette belle Espèce, desrégions Sud-Est voisines du Nyanza Oukéréwé, remarquable par sa forme globuleuse, est d'une taille moitié moindre que

1. Non Limicolaria Bourguignati, de Paladithe, in : Ann. mus. civ. Genova, 1872, p. 18, pl. I, fig. 13-1', qui est l'Opeas sracilis d'Alber's, 1860. 
celle de la nilotica; son bord columellaire, au sommet duquel on remarque une petite dépression canaliforme, analogue à celle que l'on observe chez la Limnxa canalis d'Europe, descend rectilignement sans offrir aucune trace d'échancrure ou d'éminence subachatiniforme.

Je renvoie aux Bulletins de la Société malacologique de France, pour la connaissance complète de cette Espèce.

Burtoa Remoxdi, pl. iw, fig. 1 (Bulimus Reymondi, Bourguignal, Moll. Tang., p. 13, 1885).

Cette Burtoie, que l'on rencontre fréquemment dans les régions entre le Tanganika, le Nyassa et le Bangouélo, diffère de la Kraussi (Bul. Kraussi, Pfeiffer, voir ci-après), la seule Espèce avec laquelle elle a des rapports de forme et de ressemblance, par sa taille moindre, par sa coloration, parsa spire plus courte, plus obtuse, par ses tours supérieurs occupant en hauteur la moitié de l'espace qu'occupent ceux de la Kraussi, par ses deux derniers tours plus oblongs, relativement plus développés et plus amples, par sa perforation tout à fait recouverte, par son bord columellaire descendant presque rectilignement et n'offrant pas, vers la base, d'éminence sinueuse, comme celle qui caractérise la Kraussi.

Burtoa Bridouxiana, Bourguignat, spec. nov. pl. 1 , fig. 3 .

Coq. à perforation presque complètement bouchée. Test d'une forme ovoïde-allongée, mince, 
subtransparent, sillonné, sauf sur les tours supérieurs, de striations transverses que viemnent couper, sur la partie supérieure du dernier tour, d'autres lignes spirales. Coloration composée d'une série de longues flammules transverses étroites, d'un marron-jaune s'alternant avec d'autres d'un ton noir. Spire régulière, obtusément acuminée. Iluit tours peu convexes, i croissance assez lente jusqu'au dernier. Suture peu profonde. Dernier tour convexe, oblong, n'atteignant pas les deux tiers de la hauteur. Ouverture verticale, échancrée, oblongue-allongée, fortement anguleuse au sommet, régulièrement convexe du côté externe, un peu rétrécie inférieurement. Péristome droit, aigu, à bord colımellaire rectiligne, réfléchi sousune apparence de dilatation trianguliforme très allongée, descendant en pointe jusqu'à la base de l'axe. Haut., 79 ; diam., 44; h. ouv., 44, larg., 25 millim.

Mont Kidete, entre Kondoa et Mpouapoua (Ousaghara).

Burnox sebasmia, Bourguignat, sp. nov., pl. ir, fig. 1.

Coq. de forte taille (h., 118; d., 73; h. ouv., 70, 1., 48 millim.), profondément perforée (perforation i moitié recouverte). Test ventru, oblong-ovoïde, solide, opaque, fortement sillonné (sauf les tours embryonnaires), sur la partie supérieure des derniers tours, par des striations que viennent couper d'autres striations en sens inverse, et recouvert par un tissu épidermique très fugace, orné de longues flammules d'un jaune-paille, alternées 
avec d'autres d'un marron-noir. Sur les tours supérieurs, la coloration est moins foncée et plus uniforme. Spire relativement peu allongée, régulièrement acuminée, terminée par un sommet médiocrement obtus. Sept à huit tours peu convexes et à croissance régulière jusqu'aı dernier, séparés par une suture peu profonde, tout en restant accentuée. Dernier tour énorme, ventru, tout en conservant une rotondité un peu oblongue, enfin n'égalant pas les deux tiers de la hauteur. Ouverture pen oblique, échancrée, d'une forme oblongue, fortement anguleuse au sommet, non dilatée inférieurement du còté externe, comme celle de la nilotica, mais plutòt rétrécie et offrant un contour d'une convexité oblongue-régulière. Intérieur d'un blanc-nacré passant au rose-violacé vers les contours. Péristome simple, droit, aigu. Bord colımellaire robuste, fortement dilaté, réfléchi (dilatation offrant plusieurs méplats) et présentant vers sa partie inférieure une éminence subachatiniforme donnant lieu, vers la base de l'axe, à un semblant de canaliculation, bien qu'il n'y ait aucune trace de troncature.

Vallée du Malagarazi, entre Tabora et Oudjiji.

Cette Espèce est, avec la nilotica, la plus grande forme Burtoienne. Elle se distingue de la nilotica, telle que celle-ci est figurée pl. cx, fig. 1 (seulement) dans les Novitates de Pfeiffer, par sa forme un peu moins ventrue, plus nettement oblongue, par sa spire moins obtuse, plus régulièrement acuminée et un peu plus prolongée, par ses tours 
moins convexes, par son dernier tour moins ventru, par son ouverture plus oblongue, sensiblement rétrécie inférieurement, régulièrement convexe du còté externe, et non arrondie et faisant ventre comme celle de la nilotica, par son bord colımellaire d'une forme différente : ainsi, celıi de la nilolica offre une dilatation trianguliforme très large au sommet et descendant en pointe jusqu'a la base de l'axe, tandis que, chez la sebasmia, la dilatation moins large an sommet ne descend pas tout à fait jusqu'à la base et présente, vers sa partie inférieure, un méplat aceentué, méplat correspondant à une petite éminence subachatiniforme, qui donne à la columelle un cachet tout particulier.

Burtoa Kunuss (Bulimus Kraussi, Pfeiffer, Symb. hist. Hel., III, 1846, p. 85; et Monogr. Hel. viv., II, 1848, p. 184; et Krauss, Moll. sudafr., p. 78, pl. v, fig. 4, 1848; - Limicolaria Kraussi, H. et A. Adams, Gener. shells, p. 133, 1855).

Pfeiffer rapporte à cette Espèce une Limicolaria fuscolabris de Martens qui m'est inconnue.

Cette Burtoie, abondante dans les forêts aux environs de Natal, est très bien représentée dans l'ouvrage de Krauss. C'est une assez grosse Espèce ventrue-ovalaire, à spire relativement allongée et au hord columellaire pourvu d'une légère éminence subachatiniforme.

Burton Petherichi, Bourguignat, spec. nov. (Limicolaria nilotica [pars], Pfeiffer, novitates, pl. cx, fig. 3, seulement). 
Cette Espèce, considérée jusqu'à présent comme un jeune de la nilotica, est une forme adulte, qui possède cinq tours et un dernier tour offrant, à son insertion, une légère direction descendante.

Coquille de taille médiocre (haut., 74; diam., 47; haut. ouv., 47 ; larg., 26 millim.), ventrue-ovoüde, de forme un peu obèse. Test assez mince, strié et seulement décussé sur la partie supéricure des derniers tours. Coloration marron assez uniforme avec quelques flammules transverses plus foncées. Spire obtuse, à sommet relativement plus gros que celui de la nilotica. Cinq tours d'un arrondioblong, n'atteignant pas tout à fait les deux tiers de la hauteur. Ouverture presque verticale, échancrée, d'une forme irrégulièrement oblongue, anguleuse au sommet, rétrécie vers la base, régulièrement convexe du còté externe. Péristome mince, droit. Bord columellaire réfléchi sous la forme d'une dilatation triangulaire allongée, ne descendant pas jusqu'a la base de l'axe et donnant lieu, à sa terminaison, à une espèce d'échancrure subachatiniforme.

Contrée voisine du Nyanza Oukéréwé, notamment aux environs de Boma, dans le Monéré, et de Msalala, dans l'Ouniamouézi.

\section{BURTOA LAVIGERIANA, Bourg., spec. nov. (pl, iv, fig. 2)}

Coquille perforée (perforation étroite, très profonde), d'une petite taille (haut., 48; diam., 33; haut. ouv., 33; larg., 18 millim.), ovoïde-ovalaire, 
relativement très ventrue. Test très fragile, transparent, très finement striolé (sauf les tours em. bryonnaires), décussé par de petites linéoles spirales sur la partie supérieure des deux derniers tours. Epiderme très fugace, d'une coloration jaune pâle terne, avec des flammules transverses marron foncé sur le dernier tour. Spire courte, très obtuse. Six tours convexes, à croissance lente jusqu'au dernier. Suture assez profonde. Dernier tour ventru, dépassant les deux tiers de la hauteur. Ouverture légèrement oblique, échancrée, anguleuse au sommet, rétrécie à la base, convexe du côté externe, intérieurement d'une nacre blanchàtre, passant au bleuâtre vers les contours et sur la dilatation de l'axe columellaire. Péristome très mince, fragile, droit. Bord columellaire fortement réfléchi sous la forme d'une dilatation triangulaire allongée ne descendant pas tout à fait jusqu'à la base de l'ouverture.

Vallée de la Makata (Ousaghara), Mikese dans le Moueré, au sud du Nyanza Oukéréwé. 


\section{BURTOPSIS}

Les deux Burtopsides, les seules qui me sont connues pour le moment, bien que proche voisines des Burtoies, sont des Espèces ayant l'apparence de Bulimes, grâce à leur test épais, opaque, et surtout à leur bord péristomal obtus, épaissi et patulescent, mais, par contre, en différant par leur axe columellaire présentant en avant, à leur partie supérieure, une large concavité subcanaliforme et offrant, vers leur base interne, un léger soupcon de retrait subachatiniforme.

Les Burtopsides sont remarquables, de plus, par leur grande taille et notamment par leur aspect rosacé. Chez ces Espèces, lorsque le tissu épidermique, d'un ton marron-carnéolé, est enlevé, la surface entière paraît d'une coloration rosacée, coloration qui, sur le pourtour apertural et sur la dilatation columellaire, passe à une nuance rosevineuse très intense. Il y a, au Brésil, un Bulime (le Bul. hemastomus, Scopoli) qui offre des teintes semblables.

Les formes de ce genre paraissent spéciales aux régions du centre équatorial, aux contrées des Nyanzas nilotiques, à l'Ounyanyembé et aux pays qui s'étendent entre le Nyassa, le Bangonélo et le Tanganika.

Les deux Burtopsides sont :

Bentopsis Ginacm, pl. v, fig. 1 (Bulimus Gimadi, 
Bourguignat, Moll. Giraud, p. 12, 1885). - Sud du lac Tanganika, entre Iendné et Pambété.

BURTORSIS JOUBERTI, spec. nov. (pl. in, fig. 1).

Testa rimato-perforata (perforatio aperta ac profunda), magna, oblonga, in ultimo insigniter ad dextram directa; solida, opacula, valide (prioribus levigatis exceptis) striata ac lineolis spiralibus eleganter decussata (lineola ad ultimi partem inferiorem deficientes), uniformiter carneolo-castanea, sub epidermide rosacea; - spira productooblonga, subventroso-acuminata, nihilominus ad apicem obtusa; - anfractibus 7 convexis, regulariter celeriterque crescentibus, sutura impressa separatis; - ultimo convexo-oblongo, relative mediocri, dimidiam altitudinis leviter vix superante ad dextram notabiliter verso, ad insertionem lente descendente; - apertura fere verticali, ovata, superne angulata, intus albo-margaritacea, ad margines et columellam ac super callum palatalem rosacco-vinosa; - peristomate obtuso, intus incrassatulo patulescente, ad basin magis patulo, undique rosaceo; margine columellari robusto, roseo, valide reflexo, superne in conspectu obsolete lateque concavo-canaliculato, ad latus apertura subcurvato; margine externo antrorsum leviter subundulato; - alt., 95 ; diam., 60 ; alt. ap., 48; lat., 37 millim.

Cette Burtopside, dédiée au capitaine L. Joubert, chef de la milice des missions tanganikiennes, a été 
recueillie, dans l'Ounyanyembé, aux envions de Tabora.

Cette Espèce est remarquable par le peu de développement en hauteur de son dernier tour, notablement porté du côté droit, caractère qui donne à l'ouverture une forme ovale et non oblongue, comme celle que l'on remarque chez la Burtopsis Giraudi.

On distinguera la Jouberti, de cette Espèce, à sa taille un peu plus faible, à sa forme moins ventrue, à sa spire plus élancée, moins obtuse, à sa croissance spirale plus régulière, à sa perforation non recouverte, à son ouverture ovale, non oblongue, à son bord columellaire supérieur plus largement et plus profondément concave, non rectiligne du côté apertural, mais subarqué dans un sens légèrement incliné de gauche à droite; enfin notamment, à son dernier tour plus arrondi vers l'ouverture, plus petit, dépassant à peine la moitié de la hauteur, tandis que celui de la Giraudi, de forme oblongue, dépasse de 22 mill. la moitić de la hauteur. Cette différence de taille entre le dernier tour de ces deux Espèces donne à chacun de ces Burtopsides un aspect très dissemblable. 


\section{I}

\section{I M I GOL A R I A}

Notre ami, le célèbre explorateur de Madagascar, M. Alfred Grandidier, membre de l'Institut, a donné dans les Bulletins de la Société Malacologique de France (t. II, 1885, p. 158 ì 162) une excellente étude rectificative sur les Limicolaires signalées par le $D^{r}$ Smith dans les régions équatoriales de l'Afrique.

Le $D^{r}$ Smith avait, en effet, décrit et fait figurer de ces régions :

$1^{\circ}$ Une Limicolaria Caillaudi (in : Proceed. zool. Soc. Lond., 1881, p. 284, pl. xxxıI, fig. 13) comme similaire de la vraie Caillaudi de Pfeiffer ${ }^{1}$, Espèce très exactement représentée, dans les Notitix malacologicx de Shuttleworth ( pl. vi, fig. 7-8), sous l'appellation de candidissima;

$2^{\circ}$ Sous le nom erroné de Limicolaria rectistrigata, très bien figurée (in : Proceed. zool. Soc. Lond., 1880, pl. xxxı, fig. 2), deux formes, sous ce mème nom (in : Proceed. zool. Soc. Lond., 1881, pl. xxxin, fig. 14-14 ${ }^{\mathrm{A}}$.

Or, M. Alfred Grandidier a démontré :

$1^{\circ}$ Que la soi-disant Caillaudi n'était pas celle de Pfeiffer;

$2^{\circ}$ Que les deux soi-disant rectistrigata de

1. In : Zeitschr. f. Malak., 1850, p. 86, et Monogr. Helic. viv., III, 1853, p. 385 . 
Smith étaient deux formes spéciales, non seulement distinctes de la vraie rectistrigata, mais encore dissemblables entre elles.

En conséquence, à la fausse Limicolaria Caillaudi, il a attribué le nom de Limic. Spekiana, et, aux formes soi-disant rectistrigata, à l'une le nom de Bridouxi, à l'autre celui de Burtoniana.

En somme, en tenant compte des rectifications contenues dans cet excellent travail, et en ajoutant nos propres connaissances, les Espèces de ce genre forment, pour ces contrées centrales de l'Afrique, un ensemble de douze Limicolaires, savoir :

Limicolaria Charboxyeri, Bourguignat, Spec. nov. (voir ci-après).

Environs de la mission de Kibanga, au sud de la presqu'île Oubouari (Tanganika); environs d'Itura et de Bizauda, dans l'Oukimbo, pays entre l'Ougogo et l'Ounyanyembé, sur la route des caravanes.

Limicolaria Speniana, Grandidier, in : Bull. Soc. malac. France, II, 1885, p. 160 et 162. - (Achatina [Limicolaria] Caillaudi [non Pfeiffer], Smith, in : Proceed. zool. Soc. Lond., 1881, p. 284, pl. xxxı1, fig. 13.)

Environs d'Oudjiji et de Karéma (côte orientale du Tanganika).

Limicolaria megalea, Bourguignat, spec. nov. (voir ci-après).

Plaine de Knicomba, sur la côte sud-occidentale du Tanganika. 
Limicolarta Covlboisi, Bourguignat, spec. nov. (voir ci-après).

Kérasa, dans l'Ousaghara.

Limicolarm Dromauxi, Bourguignat, spec. nov. (voir ci-après).

Environs de la mission de Kibanga (Tanganika).

Limicolaria sepulcratis, Bourguighat, spec. nov. (voir ci-après).

Vallée du Malagarazi, entre Tabora et Oudjiji.

Limicolaria rectistugata, Grandidier, in : Bull. Soc. malac. Fr., Il, juillet 1885, p. 162, et Bourguignat, Moll. Giraud Tang., p. 28, août 1885; (Achatina [Limicolaria] rectistrigata, Smith, in : Proceed. zool.Soc. Lond., 1880, p. 346, pl. xxxı, fig. 2 [bonne].

Espèce abondante dans toutes les régions sud du Tanganika, Karéma, Pambété, Mpala, etc...

Limicolama Buntoniaxa, Grandidier in : Bull. Soc. malac. Fr., II, 1885. (Achatina [Limicolaria] rectistrigata [pars], Smith, in : Proc. zool. Soc. Lond., 1881, pl. xxxm1, fig. 14.)

Région orientale du lac.

Limicolaria Budouxi, Grandidier, in : Bull. Soc. malac. Fr., II, 1885, p. 161 et 162; - (Achatina [Limicolaria] rectistrigata [alt. pars], Smith, in :

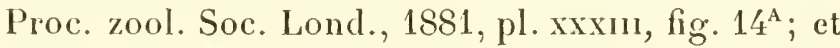
Limicolaria Martensiana [non Smith], Martens, in : Conch. Mittheil. II, Band V et VI, 1885, pl. xxxiv, fig. 1-2 [bonnes figures]).

Régions tanganikiennes, aussi bien dans les contrées occidentales qu'orientales. 
Le $\mathrm{D}^{\mathrm{r}}$ Martens (sous le nom de Martensiana) signale cette forme de la vallée du Congo, à Nyangwe.

Limicolaria Martensiana, Crosse, in : Journ. conch., 1881, p. 297, et Grandidier, in : Bull. Soc. malac. Fr., II, 1885, p. 162; (Achatina [Limicolaria] Martensiana, Smith, in : Proc. zool. Soc. Lond., 1880, p. 345, pl. xxxı, fig. 1-1 ${ }^{\mathrm{A}}$ ).

Régions dı Tanganika. Cette forme est très distincte de celle que le $D^{r}$ Martens (Conch. Mittheil., pl. xxxır, fig. 1-2) a publiée sous le même nom, comme l'on peut s'en convaincre par la comparaison des figures.

Limicolaria Giraudi, Bourguignat, Moll. Giraud Tang., p. 24, 1885, et pl. vi, fig. 5 .

Environs de Mpala, sur la côte occidentale.

Limicolaria Lamellosa, Bourguignat, spec. nov. (voir ci-après).

Presqu'ile Oubouari (Tanganika).

LIHICOLARIA CHAKBONYIERI, spec, nov. (pI. vi, fig. 7-8).

Testa punctiforme rimata, elongata, relative tumida, crassula, opacula, nitida, striatula, albescente cum flammulis castaneis (flammulæ superne angustæe, densatx, inferne conjunctæ, latæ fulguratæque); - spira elongata et attenuata, nihilominus sat obtusa, ad summum obtusiuscula; - anfractibus 9 convexiusculis, lente crescentibus, sutura sat impressa separatis; ultimo convexo-elongato, $1 / 2$ altitudinis superante, 
inferne attemuato; - apertura obliqua, elongata, transverse angustata, superne angulata, ad basin coarctata ac retrocedente; marginibus (columellari ac externo) subparallelis; - peristomate recto ac acuto; columella recta, superne bene reflexa et quasi subcanaliformi, inferne acuta; margine externo undulato, in medio antrorsum valde arcuato ; callo palatali sat crasso, albescente; - alt., 67; diam., 21; alt. ap., 26 ; lat., 10 millim.

Cette Espèce, remarquable par la disposition de ses flammules, par son ouverture allongée, fortement rétrocédente à la base, de plus, étroite, à bords (interne et externe) subparallèles, par son bord externe sinueux-ondulé et très arqué en avant à sa partie médiane, est dédiée au Rév. P. missionn. Charbonnier.

LIMICOLARIA MEGALEA, spec. nov. (pI. vi, fig. 4).

Testa obtecte rimata, perelongata, subcylindracea, opacula, crassula, obsolete striatula, circa suturam crispata, uniformiter sublutescente-albidula cum flammulis castaneis irregulariter sparsis; - spira productissima, cylindracea, lente attenuata, ad summum obtusa; - anfractribus 9 convexis, lente crescentibus, sutura profunda separatis; - ultimo convexo, 1/4 altitudinis leviter superante; - apertura obliqua, ovata; - peristomate recto, acuto; - columella mediocri, reflexa, breviter curvata; - margine externo antrorsum parum arcuatulo ac retrocedente; - callo palatali 
diaphano, fere inconspicuo ;- alt., 60; diam., 17; alt. ap., 17 ; lat., $81 / 2$ millim.

Cette belle Espèce, à cause de sa forme cylindrique très allongée, ne peut être rapprochée que de la Limicolaria Spekiana, dont elle se distingue par sa taille moindre, par sa forme plus grêle, par sa spire plus régulièrement atténuée, par ses tours plus convexes, par son ouverture et sa columelle de forme toute différente, comme on peut le voir par la comparaison des figures.

LIHCOLARIA COLLBOISI, spec. nov. (pl. vi, fig. 1).

Testa subrimata (rima fere omnino tecta), gracili, perelongata, cylindracea, ad summum obtuse attenuata, opacula, crassula, nitida, striatula, albescente cum flammulis castaneis continuis aut interruptis; - spira valde producto-elongata, gracili, attenuata, ad summum magis attenuata, nihilominus obtuse terminata; - anfractibus 9 convexiusculis, primo lente, dein sat celeriter crescentibus, sutura impressa separatis; - ultimo convexoelongato, $1 / 3$ altitudinis non xquante; - apertura obliqua, elongato-oblonga; - peristomate recto, acuto; - columella reflexa, leviter curvata; margine externo antrorsum arcuatulo; callo palatali diaphano, nihilominus sat crassulo; - alt., 61 ; diam., $15 \mathrm{1} / 2$; alt. ap., 19 ; lat., 7 millim.

Cette Limicolaire, dédiće au Rév. P. Coulbois,

1. Grandidier (voir ci-dessus), Espèce publicee par Smith, sous le nom de Caillaudi (non Pfeiffer). 
se distingue de la Limic. megalæa, la seule Espèce avec laquelle elle pourrait être assimilée, par sa forme plus grêle, par conséquent par son apparence plus élancée, bien qu'elle soit de même taille; par sa spire moins régulièrement atténuée, mais conservant sur un parcours assez long la même grosseur; par ses tours moins convexes; par sa suture moins profonde; par sa croissance spirale moins régulière; par son dernier tour plus allongé, moins convexe; par son ouverture plus longue, plus étroite, à peine convexe du côté externe.

LIMICOLARIA DROMAUXI, spec. nov. (pl. vi, fig. 3).

Testa punctiforme rimata, perelongata, attenuatocylindracea, crassula, opacula, nitida, uniformiter pallideque subalbidulo-lutescente, lievigata politaque, in inferioribus obsolete sublamellosa; spira valde producta, regulariter acuminata, nihilominus ad summum obtusiuscula; - anfractibus 10 convexis, regulariter lenteque crescentibus, sutura profunda separatis; - ultimo convexo, $1 / 4$ altitudinis leviter superante; - apertura obliqua, sat lunata, subrotundato-ovata; - peristomate recto, acuto; - columella reflexa, subrecta, margine externo retrocedente, antrorsum arcuatulo; callo palatali crassulo; - alt., 44; diam., 14; alt. ap., 13; lat., 7 millim.

Cette Limicolaire, du même groupe que les Limic. Speliana, megalxa et Coulboisi, se distingue de ces Espèces par sa taille plus faible, par sa coloration uniforme sans flammules, par son 
test lisse, poli, présentant, sur les tours inférieurs, des lamelles obsolètes, bien que nettement prononcées, par sa spire régulièrement acuminée, par sa croissance spirale plus lente, par son ouverture ovalaire assez arrondie.

LIMICOLARIA SEPULCRALIS, spec. nov. (pI. vi, fig. 2).

Testa obtecte rimata, oblongo-elongata, obtusa, crassula, opacula, nitida, subtiliter striatula aut polita, ad summum uniformiter fusco-rubescente, in cateris carulescente cum flammulis lividovinosis; - spira producta, oblonga, ad apicem obtusa; - anfractibus 8 convexiusculis, regulariter ac sat lente crescentibus, sutura parum impressa separatis; - ultimo convexo-oblongo, dimidiam altitudinis non attingente; - apertura subobliqua, elongato-angustata; marginibus (columellari et externo) fere parallelis; - columella reflexa, subrecta; - peristomate recto, acuto, intus incrassatulo; - margine externo antrorsum arcuatulo; - callo palatali nullo; - alt., 46; diam., 17; alt. ap., 20 ; lat., 7 millin.

Cette belle Espèce, qui n'a aucun rapport avec les Limicolaires précédentes, est remarquable par sa coloration bleuacée, ornée de flammules d'un ton vineux sombre, comme métallique, flammules qui, sur le dernier tour, au lieu de descendre verticalement, prennent, sur la partie inférieure, une direction spirescente, et par son ouverture très étroite, à bords presque parallèles. 
LIMICOLARIA LAHE LOSA, spec. nov. (pl. vi, fig. 6).

Testa obtecte rimata, sat parvula, oblongo-elongata, crassula, opacula, subnitidula, uniformiter luteo-straminea ac eleganter (supremis lavigatis exceptis) lamellosa (lamella regulares, obsoletæ, sat distantes, in ultimo prope aperturam evanescentes); - spira producta, oblongo-attenuata, ad summum obtusiuscula; - anfractibus 8 convexiusculis, lente crescentibus, sutura impressula separatis; - ultimo convexo, $1 / 2$ altitudinis leviter superante; - apertura subobliqua, ovata, intus albo-margaritacea; - peristomate recto, acuto; columella mediocri, reflexa; - margine externo antrorsum vix arcuatulo; callo palatali nullo; alt., 32 ; diam., 11 1/2; alt. ap., 11 1/4; lat., 6 millim.

Cette petite Limicolaire est très remarquable par sa coloration jaune-paille uniforme et par son test élégamment sillonné par des lamelles régulières, également distantes les unes des autres et très fortement prononcées. 


\section{XII}

\section{STENOGYRA}

J'ai à faire connaître deux Sténogyres nouvelles, découvertes par le R. P. missionnaire Alexandre Le Roy, à une altitude de 1800 à 2000 mètres, sur le N'gourou, massif de montagnes au septentrion de la haute vallée du Vouani, entre l'Oukamba et l'Ousaghara. Ces deux Espèces se rencontrent dans les mêmes conditions vitales que la belle Colpanostoma Leroyi, décrite ci-dessus, page 48 .

STENOGYRA LEROYi, sp. nov. (pl. vi. fig. 2).

Testa elongata, mediocriter tumida, sat tenui, subpellucida, nitida, uniformiter rubro-castanea aut subvinosa', ad basin columella pallidiore, eleganter lamellata (lamella regulares, arctx, productæe, ad summum validiores ac magis distantes, ad partem inferiorem ultimi tenuiores), ac, in ultimo ad peripheriam sulco spirali nitidiore, quasi micante, obsolete subnoduloso, circumcincta; spira elongata, acuminata, nihilominus ad summum obtusiuscula; - anfractibus 9 convexis, regulariter lenteque crescentibus, sutura bene impressa, quasi profunda, separatis; - ultimo mediocri, $1 / 3$ altitudinis superante, oblongo-convexo, inferne

1. Sur certains échantillons, la coloration est d'uue teinte pâle jannacée-subolivâtre. 
nitidiore ac nimis costulato, ad peripheriam unisulcato (sulcus filiformis, leviter productus, nitidissimus, sublrvigatus, nihilominus sub lente subnodulosus ac usque ad aperturam notatus), superne eleganter lamellato; - apertura subobliqua, elongato-oblonga, angustata, superne ac inferne angulosa, externe regulariter convexa, intus vinosa; peristomate recto, acuto; - margine columellari pallidiore, subsuccineo, recto, ad basin oblique truncato; - callo palatali tenui et diaphano; alt., 40 ; diam., 16 ; alt. ap., 16; lat., 7 millim.

Parmi les Sténogyres, je ne vois que la Stenosyra Calabarica ${ }^{1}$, des monts Cameroons, entre le Gabon et les côtes de la Guinée, qui puisse hui ressembler à cause de son mode de lamellations. Mais si les lamelles sont semblables et si le dernier tour est entouré d'un sillon phériphéral analogue à celui de la Leroyi, cette Sténogyre diffère essentiellement de la nôtre par une forme plus pyramidale, par une spire plus acuminée, par son dernier tour plus ventru, moins oblong, par son ouverture moins allongée, par son axe columellaire très court, non recto-descendant, mais très courbe et brusquement tronqué.

STENOGYRA GRANDIDIERIANA, sp. nov. (pl. vi, fig. 9-10).

Testa oblongo-elongata, solida, nihilominus subtranslucida, nitidissima, lavigata, polita (sub)

1. Martens, Moll. Buchholz in West. Afr., pl. m, fig. 5-6, $18 \% 6$. 
validissimo lente subtiliter striatula), uniformiter opalina, modo ad partem inferiorem ultimi vinosocastanea, et super anfractus (supremis exceptis) lineolis atro-castaneis, angustis, ad peripheriam in lato maculo eodem colore terminatis, eleganter ornata; - spira attenuata, ad summum obtusa; anfractibus 9 convexiusculis, regulariter lenteque crescentibus, sutura impressa separatis; - ultimo convexo, mediocri, $1 / 3$ altitudinis leviter superante; - apertura obliqua, relative parvula, irregulariter ovata, intus violacea cum maculis apparentibus, ad marginem albescente; - peristomate recto, acuto; - margine columellari brevi, curvato, ad basin quasi contorto ac abrupte valideque truncato; - callo palatali vinoso; - alt., 36; diam., $151 / 2$; alt. ap., 13 ; lat., 7 millim.

Cette belle Espèce, par le brillant de sa coloration et par la singularité de son ornementation, est une forme très remarquable, qui ne peut être confondue avec aucune des Sténogyres connues. 


\section{I II}

\section{SUBULINA}

Dans le Nomenclator Heliceorum viventium, on remarque plus d'une centaine d'Espèces classées dans ce genre. Or, lorsqu'on étudic attentivement les caractères de chacune de ces formes, on reconnait qu'une trentaine seulement possèdent les caractères propres au genre Subulina tel qu'il a été établi par Beck 1, quand cet auteur lui a assigné pour type l'Achatina cyanostoma ${ }^{2}$ du sud de l'Abyssinie. Quant aux soixante et quelques autres Espèces qu'il m'cst impossible de comprendre parmi les Subulines, je crois qu'elles doivent être réparties dans plusieurs coupes génériques différentes". Il y aurait un travail bien intéressant à faire sur les Espèces classées dans ce genre, travail trop long, qui serait ici un hors-d'œuvre, et que je laisse a entreprendre aux malacologistes qui ont plus de loisirs que moi.

Quoi qu'il en soit, en ajoutant à cette trentaine de Subulines celles qui ont été décrites cà et là dans les œuvres récentes des auteurs, on arrive à une quarantaine de Subulines pour le continent africain.

1. Ind. moll., p. 76, 1838.

2. Voir, pour la synonymie de cette Espèce, ma Malacologıe de l'Abyssinic (p. 120), 1883.

3. C'est pour l'une d'elles qu'Ancey (Bull. soc. malac. Fr., II, 1885, p. 143) a établi le genre Tomostele. 
J'ai cinq formes (trois connues et deux inédites) à signaler des contrées équatoriales de l'Afrique.

$1^{\circ}$ Subulina Mabilliaxa, Bourguignat, Malac. Abyss., p. 83 et 122, fig. 68-69, 1883.

Cette Espèce, connue seulement de l'Abyssinie, a été rencontrée assez fréquemment dans les parties boisées, sous les pierres, de la presqu'ìle Oubouari (Tanganika). Les échantillons de cette localité ne diffèrent de ceux de l'Abyssinie que par une taille un peu plus forte.

$2^{\circ}$ Subulina lenta, Smith, in : Ann. and mag. nat. hist. (sér. V, t. VI, 1880), p. 428, et in : Proceed. zool. Soc. Lond., 1881, p. 284, pl. xxxm, fig. 15.

Cette Subuline, recueillie dans les contrées nord-orientales du lac Tanganika, a été retrouvée de l'autre côté du lac, dans la presqu'ìle Oubouari. On la rencontre très rarement entière; presque toujours les tours supérieurs manquent. C'est d'après un échantillon tronqué, ne possédant plus que 11 à 12 tours, que Smith a établi la description de cette Subuline. Cette Espèce, lorsqu'elle est entière, a 17 ou 18 tours; sa spire, excessivement allongée, est pyramidale. Je donne à nouveau (pl.v, fig. 5) la représentation de cette Subuline.

$3^{\circ}$ Subulina intermeda, Gibbons, in : Taylor, New-spee. shells east coast of Afr., in : Quat. journ. conch., I, 1877, p. 282, pl. III, fig. 4.

Primitivement signalée de Zanzibar, cette Espèce a été, depuis, recueillie dans la vallée du 
Kyngani (Oukami) et dans la partie haute de la valléc du Vonami (Ousaghara).

40 SLBuliNa CILINDRACEA, spec. nov, (pl. v. fig. 2-3).

Testa perelongato-cylindrica, solidula, opacula, parum nitente, uniformiter corneo-lutescente, striatula ac prope suturam crispulata; - spira productissima, cylindrica, vix attenuata, ad summum obtusa; - anfractibus 17 convexis, arctespiratis, sutura sat profunda separatis; - ultimo mediocri, convexo; - apertura subobliqua, ovata; - peristomate recto, acuto; columella brevi, leviter curvata, inferne abrupte truncata ; margine externo recto, vix retrocedente; callo palatali crassulo; alt., 37; diam., 5; alt. ap., 5 ; lat., 5 millim.

Cette singulière Subuline, qui ressemble par sa spire à une Rhodea, vit dans les endroits boisés de la presqu'ìle Oubouari. Elle est si allongée, qu'elle est presque toujours brisće: mais, avec les fragments, il est facile de la reconstituer en son entier.

;0 SUBULINA JOUBERTI, spec. nov. (pl. v, fig. 4).

Testa gracili, perelongata, cylindrica, translucida, pallide lutescente, bene striatula, prope suturam crispata; - spira valde producta, cylindrica, parum attenuati, ad summum obtusa ac quasi mamillata; - anfractibus 12 convexis, arctespiratis, sutura profunda separatis; - ultimo minuto, convexo; - apertura obliqua, ovata; peristomate recto, acuto; columella brevi, curvata, 
abrupte truncata; callo palatali nullo; - alt., 20; diam., $31 / 2$; alt. op., $31 / 2$; lat., 2 1/4 millim.

Parties boisées de l'Ubembe, sur la côte occidentale du Tanganika.

Cette Espèce ne peut ĉtre, pas plus que la précédente, assimilée à aucune Subuline connue de l'Afrique. 


\section{XIV}

\section{CLAUSILIA}

Je donne (pl. v, fig. 8-9) la représentation de la Clansilia Girandi, dont j'ai fait comnaitre les caractères, en 1885 (Moll. Girand Tang., p. 22). Cette Clausilie, recueillie dans les anfractuosités des roches aux environs de Pambété (sud du Tanganika), est une des Espèces rares de l'Afrique. Avant sa découverte par le célèbre voyageur Victor Giraud, on ne se doutait guère qu'un représentant de ce genre, si abondant en Europe, existàt dans le centre de l'Afrique. A l'exception des régions algériennes, tuninisiennes et marocaines, qui, ainsi que l'on sait, possèdent une faune emropéenne et qui ne font pas zoologiquement partie du continent, et à l'exception également du delta égyptien, où de nombreuses Clausilies ont été constatées, on ne connaissait, en effet, que deux formes clausiliennes vraiment africaines (la sennaarica et la dystherata) spéciales aux hautes régions nilotiques. 


\section{V}

\section{A NCEYA $^{1}$}

Ce genre, établi par moi, en 1885, pour une petite coquille clausiliforme dextre possédant un pli columellaire et une palatale ${ }^{2}$, mais dépourvue de clausilium, est caractérisé par un test très allongé, orné de vastes plis comprimés, réguliers, légèrement obliques de droite à gauche, et offrant, de plus, vers la partie inférieure du dernier tour, une carène saillante, qui se produit à l'intérieur de l'ouverture sous l'apparence d'un sillon canaliforme.

Chez les deux Espèces connues, l'ouverture, très irrégulière, est anguleuse à la base du bord columellaire, vers le sommet externe près de l'insertion, et, chez une de ces Espèces (la Giraudi), à l'endroit de la carène inférieure.

Les Anceyies, d'après mes comnaissances actuelles, ne doivent appartenir ni à la famille des Helicidæ, ni à celle des Clausilidæ, mais à une famille voisine de celle des Streptostelidæ.

Axceya Giraudi, Bourguignat, Mal. Tang. Girand, p. $15,1885$.

Belle Espèce des environs de Mlilo, de forme

1. Bourguignat, Not. prod. Moll. 'Tang. Giraud, p. 14, 1885.

2. La palatale n'existe que chez la Giraudi, Espéce type du genre. 
allongée, acuminée-conoïde. Ouverture pourvue d'un pli columellaire et d'une palatale. Côté externe apertural présentant un prolongement rostriforme à l'endroit du sillon formé par la carène extérieure.

ANGEYA ADUIhABILIs, Bourg., sp. nov. (pl. vil, fig. 10-11).

Testa anguste rimata, elongata, fusiformi, fragili, pellucida, nitida, uniformiter rufo-cornea et, sicut in specie precedenti, plicis transversis productis, regulariter compressis, inter se aqualiter in direetionem leviter obliquam e dextra ad sinistram distantibus, eleganter ornata; - spira elongata, lente attenuata, ad summum obtusiuscula; - anfractibus 10-11 (superiores lævigati) arctespiratis, convexiusculis, sutura profunda ac plicis fimbriata separatis; - ultimo exiguo, superne tumidulo, inferne subattenuato, plicato (plica plicibus anfractum similes), inferue acute carinato (carina spiralis, acuto-filiformis ac prominens), et ad basin polito aut subtiliter striatulo; - apertura verticali, irregulariter angulato-oblonga (angulus notatus ad basin columella) et externe biangulato-canaliculata (canaliculus unus superus prope insertionem, alter inferus carince respondens), tandem plicata (plica unica lamelliformis ad columellam); margine columellari leviter in directionem e sinistra ad dextram oblique recto; - peristomate recto, acuto, superne incrassatulo, inferne crassiore et patulescente; - marginibus callo junctis; alt., 8; diam., 2; alt. ap., 1 1/2; lat., 1 millim.

Cette singulière Espèce, recueillie sous les 
rochers entre Mpala et Mlilo, diffère de la Giraudi par sa taille plus faible, plus fluette, par sa forme en fuseau (celle de la Giraudi est acuminée en forme de cône très allongé), par sa spire non conoïde, seulèment atténuée, à sommet moins aigu, par ses plis plus saillants et plus espacés, par ses tours un peu plus convexes, et, notamment, par son ouverture de forme toute différente (voir les figures 10 et 13), ne possédant qu'un pli columellaire et n'offrant pas ce prolongement rostriforme, qui caractérise l'Anceya Giraudi. 


\section{V I}

ENEIDE ${ }^{1}$

Dams mon llistoire malacologique de l'Abyssinie, j'émettai, en $1883^{2}$, l'opinion suivante, au sujet du genre Ennea :

"Ce genre, établi en 1855 par les frères Adams, a pour type les Pupa bicolor, Pirrici et Ceylanica. Pfeiffer $^{3}$, en l'adoptant, le divise en cind séries d'Espèces: Edenlulina, Uniplicaria, Enneastrum, Gullela et Ilutlonella. Cet auteur range, dans cette cinquième et dernière série, les Pupa que les créateurs cilent comme les types du genre, et englobe, dans les quatre premières, une suile d'Espèces qu’il présente comme les formes types des Ennea. Il est impossible de faire mieux pour dénaturer le caractère d'une coupe générique. Aussi Albers ${ }^{4}$, trompé par Pfeiffer, adopte le genre pour les formes ventrues-obovales ou obtuses, et rejette les vraics Ennea dans la seconde section du genre Gonospira de Swainson. M. Morelet ${ }^{5}$, de son còté, également induit en erreur, adopte aussi ce genre pour les Espèces ventrues-obovales et n'admet pas

1. Bourguignat, Malac. Abyss., p. 24, 1883.

2. 1'age 75 .

3. Versuch einer anordnung der Heliceen nach naturlichen Gruppen, in : Malak. Blâtter, 1855, p. 173.

4. Die Heliceen, p. 301 (2e édition, 1860 ).

5. Séries conchyl., 2e livr., 1860, p. 73. 
le Pupa bicolor, juste l'Espèce typo-générique des frères Adams. "

Je professe encore la même opinion. Ce genre a été complètement dénaturé par Pfeiffer et ses serviles imitateurs. Il convient actuellement de revenir aux principes, de rétablir ce genre, tel qu'il a été conçu et de répartir d'une tout autre facon les formes ennéennes.

\section{ENNEA}

En établissant cette coupe générique, les frères H. et A. Adams ${ }^{1}$ ont cité trois Espèces : les Pupa bicolor, Pirriei et Ceylanica, ainsi que deux autres qui ne peuvent s'accorder avec ces trois, qui sont les types du genre.

Ces trois formes, ou Espèces, sont très exactement représentées sur la planche xxxiı (fig. 12-14, Pirriei, 15-17 bicolor, 18-20 Ceylanica) du premier volume des Novitates de Pfeiffer.

Ces Espèces sont de petites coquilles allongées, cylindriques, à spire à peine atténuée, presque aussi grosse au sommet (qui est obtus) qu'au dernier tour, et ressemblant comme forme et comme apparence aux Spltyradium du sud de la France et du nord de l'Italie. Le test est brillant, transparent poli ou striolé, transparent, opalin ou à deux teintes (bicolor); les tours, presque plans, ont une croissance serrée; le dernier tour, un peu contracté, offre extérieurement une dépression scrobiculaire;

1 Genera of shells. II, p. 171, 1855. 
la fente ombilicale est fort médiocre; l'ouverture échancrée, semi-oblongue, entourée d'un fort péristome patulescent souvent très épais, comme lamelleux du côté externe (Pirriei), est oruée de deux à trois denticulations: une palatale près de l'insertion, une pariétale (bicolor, Ceylanica) remplacée chez la Pirriei par un encrassement péristomal; enfin, une collumellaire (Perriei, bicolor), qui n'existe pas chez la Ceylanica, qui, par contre, possède un petit denticule infra-apertural.

Les trois Enneas conuues sont :

Exxea bicolor, $I$. et A. Adams, Gen. of shells, II, p. 17I, 1855 (Pupa bicolor, Hutton, in : Journ. asiat. soc., III, p. 86, 1834).-1les Seychelles. Cette Espèce est bien représentée dans les Novitates de Pfeiffer et affreusement figurée dans l'allas des Pupas de Kunster (2édit. Ghemnitz), pl.xur, fig. 9-10.

Exven Prrmer, Pfeiffer, Ueber die Gatt. Ennea, in : Malak. Bl., 1855, p. 62 (Pupa Pirriei, Pfeiffer, in: Proc. zool. Soc. Lond., 1854, p. 295; Inttonella Pirriei, Pfeiffer, 1855, ete.). - Monts Khoondah, dans l'Inde.

Exnea Cerlaxica, Pfeiffer, in : Malak. Bl., 1855, p. 63 (Pupa Ceylanica, Pfeiffer, in : Proc. zool. Soc. Lond., 1855, p. 9). - Ile de Ceylan.

On n'a pas encore constaté de véritables Enneas sur le continent africain; celles que l'on a signalées, sous ce nom, apparliennent aux coupes génériques qui suivent: 


\section{PTYCOTREMA}

Les Ptycotrèmes n'ont été jusqu'à présent trouvés que sur les còtes de Guinée et du Gabon; ce sont des Espèces de forme allongée, à spire assez acuminée, à ouverture pourvue de nombreuses denticulations s'enfoncant dans lintérieur, et ressemblant à un Pupa secale d'Europe.

Prycotrema Gumaca, Bourguiguat (Cyclodontina ${ }^{2}$ Guineensis, Beck, Index. Moll., p. 88, 1838; Pupa [plycotrema] Guineensis, Morch, Cat. Joldi, p. 33, 1852; Bulimus Morchi, Pfeiffer, Bul. [2édit. Chemmitz], no 266, pl. Lv1, fig. 7-8). - Guinée.

Pтycotnema cranostoma (Bulimus [Odontostomus] cyanostomus, Pfeiffer, in : Malak. BI., 1855, p. 149, et Monogr. Hel., IV, p. 438, 1859). — Côtes de Guinée (Vieux-Calabar). - Bien représenté dans les Novilates, pl. xx, fig. 3-4.

Ptycotrema mixgens (Énnea [gonospira] ringens, H. Adams, in : Proc.zool. Soc. Lond., 1870, p. 379, pl. xxvir, fig. 15). — Côtes de Guinée (SierraLeone).

Pтусотиема muchoxatci (Ennea mucronata, Martens, Buchl. Moll. in : Monatsber. Berl. ak.

1. Morch, Catal. Joldi, p. 33, 1852.

2. Ce genre Cyclodontina, qui renferme toute sorte de formes, un des plus mal conçu de Beck, ne peut être adopté pour ces Espèces. Le type est la Cyclodontina Draparnaldi, Espèce des Antilles ressemblant à une Cylindrelle. 
Wissensch., 1876, p. 264. pl. w, fig. 8-11). - Guinée (monts Cameroon).

Ptrchotrema Elinexse (Gulella Elimensis, Chaper, Esp. et g. nouv. [ext. Bull. Soc. zool. Fr., 1885], p. 10, pl. 1, fig. 13-15). — Côtes de Guinée (Assinie).

Ptrchotrena Assmicum (Gulella Assiniensis, Chaper [Loc. sup. eit.], p. 12, pl. I, fig. 16-18). Mème Iocalité que l'Elimense.

Ptrchotrena Treich (Gulella Treichi, Chaper, Lisp. nouv. Assinie [ext. Bull. Soc.zool. Fr., 1885], p. 7, pl. xı, fig. 10-11). - Mème localité que les deux Espèces précédentes.

Etc., ete...

\section{ENNEASTRU M ${ }^{1}$}

(Syn. Enneastrum, Uniplicaria, Gulella et Huttonella de Pfeiller).

Sous les noms d'Enneastrum, Uniplicaria, Gulella et Huttonella, Pfeiffer a réparti, dans ces coupes génériques, de nombrenses Espèces qui ne peuvent être séparées, altendı qu'elles ne diffèrent entre elles que par une diversité plus ou moins grande de leurs dents aperturales.

Chez le Pupa cerea (ype des Uniplicaria), il n'y a, sans compter un denticile columellaire, qu'un seul pli palatal;

Chez le Pupa elegantula (ype des Enneastrum),

1. Pfrifler, in : Maliak. Bl., 1855, p. 173. 
on remarque, en plus, deux lamelles pariétales, sur la paroi externe, se prolongeant à l'intérieur.

Chez le Pupa capitata (type des Gulella), on observe, encore en plus, trois plis, soit 6 en tout, savoir : un columellaire, un palatal (comme chez les Uniplicaria), trois pariétaux et un basilaire;

Enfin, chez le Pupa Kraussi (type des Huttonella), on constate 4 denticules, un palatal, un columellaire, un pariétal et un au bas de louverture; seulement, chez cette Espèce, ainsi que chez les autres de cette coupe générique, les dents, qui ne se prolongent pas dans l'intérieur, sont si fortes et si ćpaisses qu'elles obstruent presque entièrement l'ouverture.

Ces quatre appellations, destinées à séparer des formes qui ne peuvent se distinguer que par un nombre plus ou moins grand de denticules, ne sont donc pas acceptables, d'autant plus que les Espèces de ces quatre coupes génériques sont bien semblables entre elles anx points de vue de la forme, de l'aspect et du galbe. Toutes ces Espèces sont des coquilles ayant l'apparence de nos Orcules du système européen.

En conséquence, comme il m'est impossible de séparer génériquement ces Espèces, je les comprends toutes en bloc, sous le nom d'Enneastrum, créé par Pfeiffer, pour rappeler leur origine générique.

Les Enneastrum sont fort abondants en Afrique, surtout dans les régions côtières. Je ne puis les 
énumérer tous, mais je vais en citer quelques-uns, tels que les:

Enneastrum denticulatum, Hamacenicum, papilliferum, Hildebrandti, Raffrayi, ete..., des régions nilotiques.

Enneastrum capilatum, Triesianum, Buchholzi, trigonostoma, complicatum, columellare, cavidens, ringiculum, pupxforme, etc..., des régions occidentales (Sénégal, Guinée, Gabon, Benguela, Angola).

Enneastrum pusillum, candidulum, Natalense, Planti, Menkeanum, Albersi, Wahlbergi, Adamsianum, Dunkeri, Gouldi, crassidens, ete., des régions méridionales (Cap, Natal, Mozambique).

Cinq Enneastrum ont été constatés dans les régions équatoriales, parmi lesquels un seulement a été retrouvé par les Pères missionnaires. C'est l'Enneastrum levigatum, Bourg. (Ennea lrevigata, Doleru, in : Proc. zool. Soc. Lond., 1865, p. 232, et Pfeiffer, Monogr. Hel. viv. v, 1868, p. 454, et Smith, in : Proceed. zool. Soc. Lond., 1881, p. 281, pl. xxxu, fig. $6^{*}$ ).

Cette Espèce primitivement découverte dans les contrées voisines du lac Nyassa, puis du sud du Tanganika, vit également dans l'Ousaghara, aux environs de Keraza.

Je ne puis admettre, dans la famille des Enneida, les genres Edentulina ${ }^{1}$, Elma ${ }^{2}$, Passamael-

1. Edentulina, Pfeiffer, 1855. - Les Espèces de ce genre sont des Streptaxidées. (Voir ci-après.)

2. Le genre Elma, établi par H. Adams (Proc, zool. Suc. 
la ${ }^{1}$, Adjua ${ }^{2}$, et encore moins le genre Faula des frères Adams 1855 (mieux Fauluxus, Schaufus, 1869), coupe générique voisine des Pupas, et le genre Diaphera d'Albers, 1850 , établi pour quelques Espèces américaines de forme cylindrelloïde.

Lond., 1866, p. 317) pour l'Ennea Swinhoei, de l'ile Formose (Adams [loc. sup. cit.], p. 317, pl. xxxin, fig. 8), ne me parait pas, à cause de la fente supéro-aperturale située au sommet du bord externe de cette Espèce, devoir rentrer dans la famille des Enneidées. Cette fente rappelle celle des Renca (Nevill, in : Proc. zool. Soc. Lond., 1880, p. 137). Cette Siriulioei, par son test cylindrique-allongé, brillant, par la forme de son ouverture édentuléc, non moins que par la grande convexité de son bord externe, convexité faisant ventre en avant, me semble se rapprocher des Acmóidées.

1. Le genre Passamaella a été créé par Pfeiffer (Malak., Bl., 18: 7 , p. 5) pour une coquille de Socotora, le Pupa Passamaiana de Petit (Journ, conch., 1853, p. 366, pl. xun, fig. 8.Ennea Passamaina, Godwin-Austen, Shells Socotr. 2 e partie, p. 808, pl Lxwm, fig. 11 [ext. : Proc. zool. Soc. Lond, 1881]), qui ne peut être considérée conme une Ennéidée. Cetle Espèce, très remarquable par la compression rentrante de son bord externo-apertural, sur lequel se trouve un denticule, faisant opposition à une lamelle columellaire, doit ètre classée dans le voisinage des Gibbus (Montfort, 1810).

2. Le genre Adjua, établi par M. Chaper (Bull. Soc. zool. Fr., 1885) pour une tres petite coquille ['́ mill.] d'Assinie (Guince), ne posssédant que trois tours et demi, me parait basé sur une Espèce non adulte qui, malgré son bourrelet péristomal et ses cinq denticules, pourrait bien n’ètre que la parlie supérieure d'une coquille inconnue. 


\section{XVII}

\section{STREPTAXIDE}

De même que pour le genre Ennea, Pfeiffer, dans ses travaux, a complètement dénaturé le sens du genre Slreplaxis de Gray, en adoptant sous ce nom six coupes génériques telles que celles des $\mathrm{Ar}^{2}$ temon, Scolodonta, Ammonoceras, Eustreptaxis, Odonlartemon et Discartemon.

Il résulte de cetle méthode que le genre Streptaxis, comprenant l'ensemble de ces six compes, disparait du rang générique pour passer à celui de famille. Il n'y a plus, par conséquent, de genre Streptaxis. Cela est si vrai que, pour remplacer ce genre, Pfeiffer a été obligé de créer un nouveau nom, celui d'Eustreptaxis, qui est inacceptable. Il n'est pas possible, en effet, de dénaturer, avec autant de sans-gêne que l'a fait Pfeiffer, la nature d'un genre.

Quant à moi qui ai pour principe le respect des genres et des formes spécifiques, je rétablis le genre Streptaxis, tel que l'a compris Gray; j'élimine, de plus, tontes les formes hylianoïdes et hélicoïdes, non dériées, pour les classer dans une famille spéciale, celle des Artemonida (voir cidessus, page 36 ), et je ne conserve dans celle des Streptaxidie, en y ajoutant celui des Fdentulina, que les gemres Streplaxis, Gonaxis et Marconia, 
sur lesquels je crois utile d'exprimer, anssi succinctement que possible. mon opinion.

\section{STREPTAXIS}

Streptaxis, Gray, in : Lond. Mag. New.-Ser.. I. 1837. p. 484. et Syn. Brit. mus.. p. 90. 1842.

Ce genre a été établi pour l'Helix contusa de Férussac pl. xxix fig. 5-6. Espéce brésilienne très bien représentée dans les “Abbiklungen » de Philippi (Helix. pl. von, fig. 1) et dans la seconde édition de Chemnitz. Helix. pl. cur. fig. 4-6). Cette coquille. non turbiniforme. mais plutot héliciforme. est caractérisée, an dernier tour. par une croissance spirale dériée de gauche à droite: par une spire non orculö̈de. ni bulinoïde. mais ressemblant à celle d'une Hélice dela série des Fruticicola; par une fente ombilicale. perforée au centre, puis se prolongeant, au derniertour. dans une direction subrectiligne, analogue à celle que l'on remarque chez l'Helix obstructa de Srrie: par un test cos. tulé en dessus: par une ourerture oblique, échancrée. semi-ovalaire ou subtrianguliforme, entourée par un bord péristomal brièrement dilaté sur tout son contour.

Les formes qui appartiennent à ce genre, sans compter le trpe contusus. sont les:

Streptaxis Funcki. Pfeiffer. Nourelle-Grenade. epistrlium Helix], Müller. Brésil'.

1. Maigré le dire de Icerch (Journ. conch., 1866, p. 3:' catte Espèce ne provient pas de Guinée. 
Streplaxis Dunkeri, I'feiffer, Brósil. Etc...

Je rapporte encore à ce genre, mais arec moins de certitude, la série des Espèces des côtes de Guinée (Liberia, Sierra-Leone, elc.) connue sous les noms de :

Streptaxis nobilis, Gray,

$$
\begin{aligned}
& \text { - Blandingiana, Leu, } \\
& \text { - rimata, Pfeiffer, } \\
& \text { - Monrovia, Dohrl, ete., }
\end{aligned}
$$

parce que ces Espèces, tout en ayant une apparence encore hélicoüde, possćdent une spire plus élancée, plus obtusément convexe, et une ouverture moins échancrée, plus arrondie. Ces coquilles streptaxidiennes rappellent un peu, par l'ensemble delenrformeventrue-globuleuse (abstraction faite, bien entendı, de la déviation spirale), les Hélices des séries turbinata, lacertarum, ete., siabondantes dans les régrions septentrionales de l’Afrique.

On voit par cel exposé que les vrais Streptaxis de Gray n’ont été, jusqu’à présent, conslatés que dans l’Amérique du Sud et sur les côtes de Guinée. Je n'en connais pas ailleurs sur le continent africain. Les Especes streptaxidiennes qui y ont été rencontrées appartiennent aux coupes génériques suivantes : 


\section{GONAXIS}

(Gonaxis, Taylor, New. Spec. east coast Afr., in : Quat. Journ. conch., I, 1877, p. 252. - Eustreptaxis (nomen, sine descriptione), Pfeiffer, in : Malak. Bl., 1877, p. 5. - Lamelliger et Oophana, Ancey, in : Naturaliste, p. 399, 1882.)

La priorité de ce genre appartient incontestablement ì M. John Taylor, qui, le premier, a fait connaitre les caractères génériques en décrivant le Gonaxis Gibbonsi (Loc. sup. lit., p. 252, pl. n, fig. 1), tandis que le nom d'Eustreptaxis, établi par Pfeiffer, comme nom de section, se trouve seulement cité dans le courant d'une phrase, non seulement sans un mot de description, mais encore sans une citation spécifique qui aurait pu mettre le lecteur, par l'examen de l'Espèce signalée, au courant de la valeur dı nom proposé. Il convient donc dorénavant de rayer le nom d'Eustreptaxis pour le remplacer par celui de Gonaxis. Quant aux formes classées dans la section des Odontartemon, elles ne sont que des Gonaxis à ouverture plus ou moins dentée.

Le genre Gonaxis a été établi par M. John Taylor de Leeds pour une petite Espèce zanzibarienne (Gonaxis Gibbonsi), turbiniforme à spire allongée, à croissance déviée de gauche à droite et à ouverture édentulée.

L'ouverture, chez les formes de ce genre, peut être édentulée ou denticulće, de même que la spire 
peut se montrer plus ou moins allongée et plus ou moins obliquement déviée, sans qu'entre le manque ou l'exagération de ces caractères, l'on puisse établir une ligne de démarcation bien nette entre les différentes séries de forme.

Les Gonaxis sont très rombreux, parmi enx je citerai les :

Gonaxis Gibbonsi, Taylor, Zanzibar.

- Mozambicensis [Streptaxis], Smith, Zanguébar.

- Welwitschi [id.], Morelet, Angola.

- turbinata [icl.], id. id.

- Troberti ${ }^{1}$ [Helix], Petit, id.

- ovatus [Streptaxis], Pfeiffer, Gabon.

- distortus [Helix), Jonas, Guinée.

- prostatus [Streptaxis], Gould, id.

- albidus [id.], Pfeiffer, id.

- kirki [id], Dorkn, Zanguébar,

Etc., etc.;

Et les Gonaxis gibbosus [Streptaxis], Pfeiffer; Candeanus [Helix], Petit; glaber [Streptaxis], Pfeiffer; comboides [Helix], d'Orbigny; dejectus [Helix], Petit; Deplanchei [Streptaxis], Drouët, ete., ete., de l'Amérique du Sud;

Et les Gonaxis pellucens [Streptaxis], Pfeiffer; exacutus [Streptaxis], Gould; bulbulus [Ennea], Morelet; Mouhoti [Streptaxis], Pfeiffer; aberratus [Helix], Sonleyet, etc, etc., de I'Asie méridionale.

1. 'Type de la seclion des Lamelliger, l'Ancey. 
Ces citations suffisent pour montrer que toutes ces Espèces à ouverture denlée ou édentulée, à spire plus ou moins turbince, rentrent bien daus une mème coupe générique, sans que l'on puisse domner à celles que l'on voudrait constituer pour marquer une ligne de démarcation plus qu'une valeur secondaire, éest-à-dire une valeur de série.

J'ai, enfin, à mentionner deux Espèces, les plus grandes du genre, qui mériteraient, à cause de leur aspect tout particulier, l'honneur d'une série spéciale. Ces deux Espèces des régions équatoriales, sont les :

Goxaxis Cruvext (Streptaxis Craveni, Smith, in : Proc. zool. Soc. Lond.. 1881, p. 280, pl. xxxu, fig. 5). - Monbas, Pangani et sur les collines entre les bassins du Vouami et du Kingani, avant d'arriver à Kondoa (Ousaghara).

GOXAXIS BLOYETI, spec. nov. (pl. vir, fig. 1-3).

Testa anguste perforata, streptaxiformi, ventrosoturgida, curta, obesa, fere aequaliter alta quam lata, tenui, subtranslucida, nitida, lactescente, in ultimo polita, in cateris (embryonalibus exceptis) plicosa (plica circa suturam validiores erectreque ac in ultimo ab initio usque ad aperturam in punctulis pliciformibus modo super suturam se permanentes); - spira e sinistra ad dextram obliqua, perobtusa, superne compressa, vix convexa, quasi oppressa: - anfractibus 6 valde irregularibus (superiores exigui, normaliter arctespirati; medianus irregularis, sinistrorse quam ad dextram altior; ultimus 
relatire maximus, oblique e sinistrorsa ad dextram (leflectus), acsutura impressula separatis; - ultimo amplo, oblique deflecto, super regionem columellarem complanato, inferne cirea perforationem angulato; - apertura sat retrocedente, lunata, oblique semi-rotundata; - peristomate incrassato, ad columellam valide reflexo, inferne bene patulesconte, externe minus patulo ac ad insertionem fere recto; callo palalali inconspicuo; - alt., 17 ; diam., $151 / 2$; alt. ap., 10; lat., 8 millim.

Sous les détritus, dans la chaine monlueuse entre l'Ousaghara el l'ougogo.

Celte Espece se distingue du Gonaxis Croveni par sa laille moindre, par sa spire tout à lait écrasce, au point que les tours supérieurs, plus exigus et plus serpés, sont a peine visibles de lace; par sa croissance spirale plus irrégulière; par son dernier tour plus développé en hauteur, et par son ouverture moins obliquée du côté droit et par cela même de forme diflérente.

\section{MARCONIA ', nov, gen.}

Espèces de forme orculö̈de de la série de l'Orcula dolium, avec une ouverture bulimoüde ou plutòt édenfulinoüde, sans denticulations.

Spire lurbinée très obtuse, à sommet arrondi en forme de dòme. Croissance spirale normale (Mar-

1. En l'hounene da voyagen français, Ernest Marcon, de I'aris. 
conia recta, vitrea), ou bien un tant soit peu streptaxoïde), c'est-à-dire ayant une apparence très faiblement déviée, à partir du dernier tour (Marconia lata, gibbosa, enneoides). Suture toujours ornée, sur les tours médians, de petits points pliciformes. Dernier tour offrant, au-dessus de l'ouverture, un aplatissement plus ou moins prononcé, aplatissement similaire à celui des Hypocystis, s'obliquant de droite à gauche et précédé d'une gibbosité ou d'un gonflement anfractural, souvent très accentué (Harconia gibbosa). Ouverture semiovalaire, entourée par un bord péristomal bien évasé. Ombilic réduit à l'état de fente. Test brillant, transparent, cristallin, opalino-lactescent et presque poli.

Marconia lata (Ennea lata, Smith, in : Proc. zool. Soc. Lond., 1880, p. 347, pl. xxxı, fig. 4-4 $4^{\mathrm{A}}$. Espèce à croissance spirale un tant soit peu déviée à partir de l'origine du dernier tour. Aplatissement supéro-apertural sensible avec une gibbosité peu accentuée ${ }^{1}$. Cette Mareonic, des contrées qui avoisinent le Tanganika, parait abondante également dans l'Ousaghara, aux environs de Kondoa, de Kitete, de Mponapoua.

Marcosia vitrea (Ennea vitrea, Morelet, Moll. Welw., 1868, p. 84, pl. 11, fig. 3). Côtes oceidentales de l'Afrique. M. Morelet a parfaitement remarqué l'aplatissement du dernier tour, " ultimus latere aperturali subplanulatus ».

1. Ce caractère n'a pas élé saisi par le docteur Smith. 
Marconta exreologe (Streptaxis emeoides, Martens, Hildebr. cone., in : Monatsher. akad. Wiss., Berlin, 1878, p. 295, pl. 11, fig. 5-6). Espèce remarquable par la grande obliquité de son ouverture. Vallée du Pangani, dans l'Oukamba, au nord de l'Ousaghara.

MARCONLA GIBBOSA, spec. nov. (pl, vi, fig. 6-7).

Testa anguste rimata (rima superficialis, recte clongata), ovato-ventrosa, turgida, ad summum perobtuse rolundata, vitracea, nitida, opalina, fere omnino polita, in medianis circa suturam punctulis pliciformibus erectis munita ; - spira leviter streptaxiformi, obesa, curte subturbiniformi, superne perobtuse tholoidaca (apex minutus, levigatus); anfractibus 6.7 tumidis convexiuseulis, irregulariter crescentibus (superiores exigui, arcte regulares; medianus irregularis, sinistrorse altior quam ad dextram; ultimi majores sat regulares), sutura impressa separatis; - ultimo $1 / 3$ altitudinis superante, irregulariter convexo, super regionem columellarem notabiliter e dextra ad sinistram oblique complanato ac ante complanationem gibboso (gibbositas obsoleta, nihilominus prominens), tandem inferne circa rimam umbilicalem subangulato et ad basin prope marginem aperturalem leviter substrangulato; - apertura mediocriter obliqua, valde lunita, semiovata; - peristomate incrassatulo, undique expanso; callo palatali fere inconspicuo; alt., 14; diam., 8 ; alt. ap., 7; lat., 5 millim.

Sous les détritus entre Kondoa et Mpouapoua 
(Ousaghara), ainsi que dans l'Ougrogo, entre Dyaza et Ibahi, le long du chemin des caravanes.

Cette Espice ne peut ètre rapprochée que de la Marconia lata (Ennea lata, de Smith), dont elle se distingue par sa forme plus courte, plus obèse et plus rentrue, par son ourerture de forme dillérente, et nolamment par son dernier tom gibbeux, caractérisé par un aplatissement beaucoup plus accentué.

MARGONIA RECTA, spec. nov. (pl. vir, fig. 'x-ö).

Testa angुuste rimata rima superficialis, sinuose elongata), turbiniformi, oblongo-ventrosa, ad summom perobtuse rotundata, vitreo-latescente, nitidissima, polita, in medianis circa suturam punctulis pliciformibus in ultimo evanescentibus munita; - spira recta, non streptaxiformi, relative sat producta, subcylindrica, ad summum tholoïdaca; - anfractibus 7 convexiusculis, regulariter crescentibus, sutura impressa separatis; - ultimo $1 / 3$ altitudinis leviter superante, convexo-oblongo, super aperturam subcomplanato, inferne prope rimam obscure subangulato; - apertura subobliqua, lunata, semiorata; - peristomate incrassatulo, undique cilatato; callo palatali inconspicuo; - alt., 15; dian., 7 ; alt. ap., 6; lat. 5 millim.

Cá et lí dans l'Ousaghara et l'Ougogo, ainsi que dans la forèt M'gounda Mkali (Oukimbou), le long du chemin.

Cette Marconic est celle qui s'éloigne le plus de la forme typique des Streptaxidés, gricice à son 
axe columellaire parfaitement rectiligne et n'aceusant aucune dévialion. Je ne vois guère que la Marconia vilrea (Ennea vitrea de Morelet) qui, à cause de son axe également rectiligne, pourrait Ini ètre comparée, si celte forme état plus allongée, moins obtuse et moins ventrue.

\section{EDENTULINA}

Pfeiffer, Vers. anordn. Melie, in : Malak. BI., 1855 , p. 173, el Monogr. Hel. vir., IV, p. 335, 1859, et VII, 1876, p. 498).

Classée jusqu'à présent parmi les Enneas, cette coupe générique doil être rangée dorénarant dans la famille des Streptaxiclées.

Ce genre comprend des Espèces oroüles plus ou moins oblongues, ayant un aspect pupoüde, tout en possédant une apparence bulimoüde.

Ces Especes, d'un galbe tout particulier, sont caracterisces par un test tramsparent, poli on très finement strié, reconvert par un tissu épidermique excessivement ténu, d'une nuance opaline plus ou moins pure; par une spire obtusément atténuée, à croissance spirale normale (saul ehez la Grandidieri); par un dernier tour bien développé, convexe, oflrant, en dessus de l'ouverture, un très léger aplatissement streptaxidien, généralement peu accusé, néanmoins toujours bien saisissable à première vue; par une perforation étroite, souvent réduite à une fente; par une ourerture peu oblique, échancrée, semi-ovalaire, édentulée, en- 
tourée par un péristome brillant, épaissi et également évasé sur tout son contour; enfin, pourvue d'un axe columellaire, sans dents, largement dilaté, sous une forme obliquement triangulaire d'une apparence toute spéciale.

Les Espèces, que je considère comme édentulines, sont les suivantes:

Edextulixa ovouna (Bulimus ovoideus, Bruglière, in : Encyel. méth., vers, I [ $1^{\text {re }}$ partie, 1789], P. 335; Pupa grandis, Pfeiffer, Symb. Helic., III, 18'6, p. 95, et Pliclippi, Abbild., II, XII, p. 156 , Bul., pl. vi, fig. 4).

Cette Espèce, la plus grande des Édentulines, est bien figurée (sous le nom de Bulimus grandis, Deshayes) dans l'ouvrage de Férussac, pl. cxus, fig. 1-2, et dans la seconde édition de Chemnitz (Pupa), pl. xvı, fig. 2-3.

Madagascar, Socotora. - Ousaghara au mont I'gourou, à une altitude de 2000 mètres.

Edentulixa ixsignis (Ennea insignis, Pfeiffer, Novit., I, p. 112 [1859], pl. xxxu, fig. 1-2). - Le Gabon. C'est la plus ventrue et la plus obtuse Édentuline.

Edextulina tumad (Ennea tumida, Morelet, in : Sér. conch., II, 1860, p. 75, pl. v, fig. 7). Nayotte.

Edentulisa intemedia (Pupa intermedia, Morelet, in : Rev. zool., 1851, p. 220, et Ennea intermedia, Morelet, in : Sér. conch., Il, 1860, p. 76, pl. v, fig. 6). -- Madagascar. 
Eoextulixa obesa (Buliminus obesus, Taylor, Shells east coast Afr., in : Quat. journ., I, 1877, p. 255, pl. 11, fig. 3; Ennea zanguebarica, Morelet, in : Journ. conch., 1889, p. 6, pl. ı, fig. 7). - Ile de Zanzibar. Còtes du Zanguébar à Mélinde, Monbas, Tanga, Quiryana, ete. Monts N'gourou, au nord de l'Ousaghara.

Edextulisa bulanfonus (Ennea bulimiformis, Grandidier, Moll. Ousagh., in : Bull. Soc. malac. Fr., IV, 1887, p. 188). - Collines montueuses entre l'Ousaghara et l'Ousegona.

Edextulisa munoll (Pupa minor, Morelet, in : Rev. zool., 1851, p. 220, et Ennea minor, Morelet, in : Sér. conch., II, 1860, p. 77, pl. v, fig. 11). Madagasear.

Edextrlixa axodox (Ennea anodon, Pfeiffer, in : Proc. zool. Soc. Lond., 1855, p. 100, et Norit., I, p. 59, pl. xvit, fig. 5-6). - Mayotte, Maurice.

Edextulaxa Liberiaxa (Bulimus Liberianus, Lea, in : Pfeiffer, Monogr. Hel., II, 1848, p. 116; Bulimus pupulus, Horelet, in : Rev. zool., 1848, p. 352; Ennea Liberiana, Pfeiffer, 1859).-Guinée, Gabon.

Edextulisa anexicola (Ennea arenicola, Morelet, in : Sér. conch., II, 1860, p. 80, pl. v, fig. 9). Madagascar. C'est la plus petite Espèce du genre.

J'ai, enfin, a faire comnaitre une nouvelle forme découverte dans le Nord de l'Ousaghara, à une altitude de 2000 mètres, sur les monts N'gourou. 
EDENTULINA GRAXIDIDIERI, spec. nov. (pl. vis, fig. 8-!).

Testa anguste rimata (rima profunda), oblonga, relative mediocriter tumida, inferne subattenuata, nitida, subtranslucida, sat fragili, polita aut subtiliter substriatula, uniformiter sublutescente-opalina: - spira sat oblonga, attenuata, nihilominus ad summum obtusa; - anfractibus 7 convexiusculis, regulariter ac sat lente usque ad ultimum, dein subirregulariler crescentibus (ultimus ab initio sat velociter descendens, dein ad aperturam notabiliter ascendens), et sutura impressula separatis; - ultimo $4 / 3$ altitudinis superante, convexo, inferne subattenuato, super aperturam leviter subcomplanato, ad insertionem valide ascendente; apertura non retrocedente, sed verticali ac potius antrorsum ad basin provecta; - peristomate incrassato, undique expanso ac reflexiusenlo; - coIumella reflexa, recliuscula; margine exlerno arcuato; callo palatali tenuissimo; - alt., 18; diam., 11; alt. ap., 11 ; lat., 7 millim.

Espèce remarquable par son ouverture verticale dont la base est sensiblement portée en avant, at lieu d'être rétrocédente; par la croissance spirale de son dernier tour, d'abord descendante à son origine, puis ascendante près de l'ouverture.

J'ajonterai que lorsqu'on regarde de profil cette Édentuline, le contour dorsal, par suite de la projection en avant de l'ouverture, parait plus bombé que le contour facial. 


\section{XVIII}

\section{GYCLOSTOMID A}

Les Espices de la grande famille des Cyclostomidés, constatées de Mogguedonchou à la contrée de Bagamoyo, et de cette contréc anx régions tanganikienne, appartiennent aux genres suivants :

\section{GEORGIA}

(Bourguignat, Moll. Comalis de la Mission Revoil, p. 65, 1882.)

Ce genre, élabli sous ce nom ${ }^{1}$, aux dépens du Otopoma, est caractérisé par des Espèces possćdant un ombilic entièrement recouvert par l'expansion du bord columellaire, expansion qui se développe uême souvent sur une grande partie du tour inférieur.

Georala xatıcopsis, Bourguiguat, Moll. Comalis Revoil, p. 71, pl. 11, fig. 43-48, 1882.

Environs de Meurka, entre Brava et Moguedouchou. L'unique échantillon très adulte, recueilli daus cette localité, est remarquable par l'épaisseur el l'encrassement de son bord péristomal.

On rencontre également, non loin de Menrka, dans les dépôts récents de la vallée de l'Ouébi,

1. Prénom du voyageur Revoil. 
cette Espèce, ainsi que la suivante, à l'état fossile.

Georgia guldaix, Bourguignat, Moll. Com. Revoil, p. 69 et 72, pl. ı1, fig. 49, 1882 (Cyclostoma Guillaini ${ }^{1}$, Petit, in : Journ. conch., 1850, p. 51, pl. 1v, fig. 3).

Très abondante dans toute la vallée de l'Ouébi depuis Guélidi jusqu'aux environs de Meurka et de Moguedouchou.

Les échantillons de ces pays ont tous le péristome plus fortement bordé et plus épaissi que ceux de la région du cap Gardafui.

Geongia Perrieni, Bourguignat, Moll. Com. Revoil, p. 73, pl. 11, fig. 50-51, 1882 (Otopoma Perrieri, Bourguignat, Moll. Com. Medjourtin, p. 4, 1881).

Environs de Meurka. Peu abondante.

Georga Polrien, Bourguignat, Moll. Com. Revoil, p. 74, pl. 11, fig. 54-56, 1882 (Otopoma Poirieri, Bourguignat, Moll. Com. Medj., p. 6, 1881).

Meurka.

Geongra Revolu, Bourguignat, Moll. Com. Revoil, p. 76, pl. 11, fig. 52.53, 1882.

Cette Espèce, découverte à Fararalé, chez les Comalis Dolbohante, n'a été retrouvée qu'à l'état fossile dans les dépôts de l'Ouébi.

1. Non Olopoma Guillaini, de Pfeiffer, qui est une Rochebrunia. 


\section{ROCHEBRUNIA}

Bourguignat, Moll. terr. flur. recueillis en Atr., daus le pays des Comalis Medjourtin, p. 7. 1881.)

Ce genre, également établi aux dépens des Otopoma, comprend une séric d'Espèces remarquables par leur forme turbinée-conoïde, ordinairement aussi haute que large, caractérisées, en outre, par des tours sphériques bien bombés, à croissance normale, dont le dernicr n'égale pas, sauf chez quelques Espèces, la moitié de la hauteur, et par un bord columellaire médiocrement dilaté, ne recousvant jamais l'ombilic et ue possédant pas cette saillie anguleuse qui distingue celui des Otopoma '.

Dans le numéro du Journal de conchyliologie, en date du $1^{\text {er }}$ octobre 1884 , bien que ce numéro ait paru le 15 avril 1885?, un des directeurs de ce Journal a émis l'opinion (p. 364)que le genre Rochebrunia était sans valeur, et cette même personne, à quelques pages plus loin (p. 370), dans le même article, a établi,pour une des Rochebrunies, le nouveau genre Guillainia ${ }^{3}$. Quelle logique! - Ou le

1. Celle saillie a été parfaitement comprise et représentée par M. Godwin-Austen, sur la pl. xxvu de ses Mollusques de lîle de Socotora (voir Proc, zool. Soc. Iond., 1881).

2. Voir ce que jai affirmé à ce sujet, page 28.

3. Non Guillainia (Bourguigual, Itelixarionidées, p. 16, janvier 1885 , genre différent, dont je viens de changer le nom en celui de Bloyerna, pour éviter tonle confusion [voir page 28]). 
grenre Rochebrunia est bon, ou il est mauvais. S'il est bon, il doit être conservé; s'il est mauvais, il doit être rejetć. Or, pour quel motif ce monsieur, qui le trouve manvais, le reconstitue-t-il pour une des Espèces, sous un nouveau nom générique ${ }^{1}$ ? C'est tout simplement absurde. Cette absurdité montre jusqu'à quel point la haine, qui, comme la faim, est toujours mauvaise conseillère " malesuada fames " peut oblitérer le jugement.

Les Rochebrunies sont les suivantes:

Rociebruxia Gullainopsis, Bourguiguat, Moll. Com. Revoil, p. S1, 1882 (Cyclostoma Guillaini [non Petit ${ }^{2}$ ], Pfeiffer, Cycl. $2^{\circ}$ édit. Chemnitz, $n^{\circ} 534$, pl. xxxry, fig. 7-8, 1853).

Beaux échantillons aux environs de Guélidi et en descendant l'Ouébi jusqu'à Meurka.

Rochebruna Delmaresi, Ancey, in : Bourguignat, Moll. Nyanza-Oukér. in : Bull. Soc. malac. Fr., IV, 1887, p. 269).

Le type de cette Espèce se tronve sur les collines de la côte occidentale du Nyanza Oukéréwé. Aux environs de Kingueni (Ouzaramo), on a recueilli une forme différant du type par sa taille un peu plus faible, par son test plus coloré, et notamment par des sillons spiranx entourant, au-dessous des tours embryonnaires, les tours médians.

Rochebruxia insularis, Bourguignat (Cyclos-

1. Voir à ce sujet les justes réflexions de M. Ancey (Bull. Soc. malac. France, II, p. 147 et 148, 1885).

2. Le Cycl. Guillaini, de Petit, est une Georgia. 
toma insulare', Pfeiffer, in : Proe. zool. Soc. Lond.,

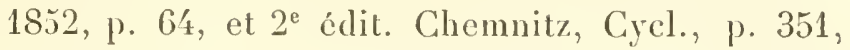
pl. xuv, fig. 5-6; - Cyclostomus insularis, Pfeiffer, Consp. Cyclost., p. 63, 1852).

Cette Espèce paraît spéciale aux régions orientoocéanniennes. Découverte d'abord dans la contrée de Natal, elle a été, depuis, recueillie dans la vallée de I'Ouébi à Guélidi, puis à Meurka, à Mélinde et à Monbas.

Rochebrunia zaxguebarica, Bourguignat (Cyclostoma zanguebaricum, Petit, in : Journ. conch., 1850 , p. 53, pl. ıा, fig. 5; - Cyclostomus zanguebaricus, Pfeiffer, Consp. Cycl., nº 322, 1852).

Espèce très abondante dans l'île de Zanzibar, oì on la rencontre le plus souvent sur les troncs des arbres et des arbustes. Vallée de l'Ouébi, près de Guélidi. - Environs de Pangani, entre Tanga et Sadani.

Rochebruxia obtusa, Bourguignat, Moll. Com. Medj. p. 7, 1S81, et Moll. Com. Revoil, p. S5, pl. 1v, fig. 60-62, 1882 (Otopoma? obtusum, Pfeiffer, in : Malak. BI. 1862, p. 202).

Primilivement recueillie à Zanzibar, puis au eap Gardafui, cette Rochebrunie a été retrouvée à l'état fossile dans les dépôts récents de la vallée de l'Ouébi, entre Meurka et Guélidi.

1. Non Cyclostoma iusulare de Smilh (in : Proc. zool. Soc. Lond., 1881, pl. xxxn, fig. 1-1 A) qui est le Cyclostoma Cambieri, Bourguignal (voir ci-après page 150 ). 


\section{A IZANIA}

Cette nouvelle coupe générique, à laquelle j’attribue l'appellation de Maizania, en l'honneur de cet infortunć enseigne de vaisseau, M. Maizan, qui, le premier de tous les explorateurs, entreprit, en 1845 , le voyage de Bagamoyo an Tanganika. et qui périt làchement assassiné à l'entrée de l'Ousaghara, est un genre établi pour une Espèce que je ne puis classer dans aueun des genres de Cyclostomidés qui me sont connus.

Cette Espèce, qui provient des sommités (vers $2000 \mathrm{~m}$.) des monts N'gourou, au nord de l'Ousaghara, vit sur les vieux bois pourris des vallons frais et ombragés de ce massif de montagnes, si riche en Espèces spéciales.

Cette coquille, remarquable par sa forme globıleuse, rappelant un peu celle d'un assez grand nombre de Cyclostomidés, se spécialise notamment par un test lisse, recouvert d'un enduit épidermique olivatre transparent, bien que relativement épais. Ce test comme membraneux, sans parties calcaires, sauf quelques-unes sur l'épaississement péristomal, parait bleuattre lorsqu'on l'examine, par transparence, à travers l'ouverture.

Son opercule (pl. vir, fig. 14-15) d'une teinte succinée, mince et transparent comme une pelure d'oignon, offre extérieurement une surface arctéspirescente faiblement concave, avec un point central autour duquel se développent de nombreuses 
spirales, qui disparaissent vers la périphérie.

Il y a, parmi les Cyclostomidés, de nombreux genres, tels que les Cyclotus, Opisthoporus, Cyclophorus, Leptopoma, Dermatocera, ete., chez lesquels l'opercule est arctéspirescent, mais je n'en connais pas qui possèdent un opercule aussi délicat et aussi fragile; de plus, tous les opercules de ces genres, relativement épais et de substance calcaire, offrent une face interne différente. Chez l'opercule de la Maizanie des monts N'gourou, la surface interne, légèrement bombée, ressemble à un glacis miroitant, sur lequel on n'apercoit aucune évolution spirale, sauf celles que l'on percoit par la transparence.

\section{MAIZANIA OLIVACEA, spec. nov. (pl. NI, fig. 14-18)}

Testa anguste perforata, globosa, parum turbinata, membranacea, subpellucida, polita, nitidula ac epidermide olivaceo uniformiter induta; - spira breviter acuminata, nihilominus ad summum obtusiuscula; - anfractibus 5 convexis, celeriter crescentibus, sutura profunda separatis; - ultimo magno, amplo, dimidiam altitudinis leviter superante, rotundato ac ventroso; - aperlura obliqua, spharica, intus cœrulescente; - peristomate continuo, recto, acuto, intus leviter albo-labiato; margine columellari subpatulescente ac leviter subrectiusculo; - alt. et diam. aque, 12 ; alt. ap., $61 / 2$; lat., 6 millim.

L'enduit épidermique qui recouvre le test de 
celle coquille n'existe pas chez les Otopoma, les Georgia et les Rochcbrunia.

\section{CYCLOSTOMA}

Les Espèces qui suivent sont de vrais Cyclostomes, dont elles ont le port et l'aspect. La structure de leur test n'est pas celle des Georgies ni des Rochebrunies, mais est tout à fait identique à celle des Cycl. sulcatum et Olivieri du bassin méditerranéen. Toutes sont fortement sillonnées de stries spirales saillantes et bien accentuées. L'anceps est parfois lisse a la partie moyenne du dernier tour, mais le plus souvent il est vigoureusement sillonné.

Crclostoma axceps, Martens, Hildebr. conch. in : Monatsb. wiss. Berlin, 1878, p. 288, pl. 1, fig. 4 .

Cette belle Espèce, signalée de l'Onkamba à Taita, pays au nord de l'Ousaghara, a été retrouvée, dans cette dernière région, dans tout le Makata, vallée arrosée par un affluent du haut Vouami, ainsi qu'à Guiriyana, localité du Zanguébar, dont je ne puis me rendre compte de la position exacte, par suite de la défectuosité de mes cartes. L'anceps est très fidèlement représenté dans le travail du $\mathrm{D}^{\mathrm{r}}$ Martens.

Crclostoma Cambieri, Bourguignat (Cyclostoma insulare [non Pfeiffer], Smith, in : Proc. zool. Soc. Lond., 1881, pl. xxxı, fig. 1 et $1^{\mathrm{A}}$ ). 
Le $D^{r}$ Smith a réuni sous le nom erroné d'insulare : $1^{\circ}$ l'insulare de Pfeiffer, Espèce à test opaque, lisse et brillant, sillonné seulement de rayons spiraux autour de la suture. Cette forme rochebrunienne paraît préférer les régions maritimes; $2^{\circ}$ le lineatum de Pfeiffer, vrai Cyclostome sillonné de stries spirales, de forme globuleuse-conoüde, à spire turbinée, etc.; $3^{\circ}$, enfin, une Espèce, à laquelle j'applique le nom de Cambieri. Ce Cyclostome diflère du lineatum, la seule coquille avec laquelle il peut être raisonnablement assimilé, par sa forme plus dépriméc, moins turbinće, plus large que haute', par son ombilic plus dilaté, par sa spire moins proéminente, par son dernier tour plus ample, plus développé, notamment dans le sens transversal, ce qui fait que ce tour dépasse de beatcoup l'avant-dernier tour, par son ouverture plus ouverte, plus portée à droite.

Cette Espèce, dédiée au brave capitaine Cambier, de Zanzibar, a été recueillie dans l'Ousaghara, l'Ounianyembé, I'Outongoué, notamment à Nyantaga, station des caravanes en avant d'Oudjiji.

Crclostom lineatum, Pfeiffer, in : Proc. zool. Soc. Lond., 1852, p. 65, et in : $2^{\mathrm{e}}$ édit. Chemnitz, p. 350, pl. xLv, fig. 3-4.

Cette Espèce, répandue ça et là sur la côte orientale, particulièrement dans l'île de Monbas, au cap Gassi et au raz Kinbiji, au sud de Dar-es-Salam,

1. Le linealum est aussi haut que large, ainsi que la liochebrunia (Cyclostoma) insularis. 
est commune aux alentours de Kondoa (Ousaghara).

C'est it tort que le $\mathrm{D}^{\mathrm{r}}$ Smith a confondu ce Cyclostome avec la Rochebrunia insularis. La figure du lineatum, donnée dans la seconde édition de Chemnitz, est exacte.

Cyclostoma Letouracxi, Bourguignat (Rochebrunia Letourneuxi, Ancey, in : Bull. Soc. malac. Fr., IV, 1887, p. 270, et Grandidier (loc. sup. cit.), IV, 1857, p. 190.

Petite Espèce se distinguant du lineatum par son ombilic plus étroit, par sa spire exiguë, par un plus grand développement de son dernier tour, ce qui rend les tours supérieurs disproportionnés en taille et en grosseur, par son ouverture plus ouverte, bien plus ample que celle du lineatum. Ce Cyclostome a été recueilli aux alentours de Bagamoyo et de Kondoa.

Crclostoma Leroy, Bourguignat,pl.vi, fig.19-20.

Testa anguste perforata, turbinata, sat tenui, subpellucida, nitida, uniformiter rubella aut lutea cum zonulis castaneis, spiraliter lirata aut subpolita cum sulcis spiralibus circa perforationem; spira producta, acuminata, ad summum obtusiuscula; - anfractibus 5-6(superiores relative exigui), celeriter crescentibus, sutura sat profunda separatis; - ultimo magno, rotundato, dimidiam altitudinis non attingente; - apertura verticali, sphxrica, superne angulata; - peristomate continuo, recto, acuto, ad marginem columellarem vix sub- 
patulescente; - alt., 14; diam., 11; alt. ap., $61 / 2$; lat., $51 / 2$ millim.

Cette Espèce, du massif montueux du N'gourou, se distingue des Cycl. lineatum et Letourncuxi, les seules coquilles qui puissent hi être assimilées, par sa forme générale plus haute, plus turbinée, par son dernier tour moins gros dans le sens transversal, par sa perforation bien moins ouverte, etc. 


\section{XIX}

\section{LIMNAID E}

Les Limnéidées, que j’ai à signaler, appartiennent aux genres Limnxa et Physopsis. Je ne connais pas, jusqu'à présent, de Physes dans les régrions qui s'étendent de l'océan Indien au Tanganika, bien qu'il doive en exister, puisqu'on en a constaté plusieurs formes dans ce lac, formes que j'ai fait figurer (pl. I, fig. 24-27) dans mon Iconographie des Animaux Mollusques du Tanganika.

\section{LIM N IEA}

J'ai domné, en 1883, dans ma Malacologie de l'Abyssinie (p. 88), la liste des Limnées africaines connues à celte époque. Cette liste, sans compter les Limnées du Système européen de la région nord du continent (Maroc, Algérie, Tunisie), élevait le nombre des Espèces africaines à vingt-neuf. Depuis, j'ai fait connaitre, dans mes Mollusques du Choa (1885) et dans mon Iconographie des Animaux Mollusques du Tanganika (1888), quatre nouvelles formes, les Limnxa Soleilleti, Gravieri, Jouberti et Lavigeriana.

Voici cette liste! :

1. Voir pour l"intelligence de cette liste la classification des Limnćes du système européen daus l'Histoire malacologique du lac Balaton, en Hongrie, par le 1) Serrain. 1 rol, in-8, 1881. 


\section{STAGNALIANA}

Limnar Caillaudi, Bourguignat, Abyssinie, Ėgyple.

- Jouberti, Bourg., Tanganika.

\section{EASERTIANA}

Limnaca exserta, Martens, Abyssinie, Égyple.

- Perrieriana, Bourg., pays Comalis.

- Poirieri, Bourg., pays Comalis.

- Revoili, Bourg., pays Comalis.

\section{BIFORMIANA}

Limmaca acroxa, Bourg., Abyssinie, Égypte.

- Cameroni, Bourg., Zanguébar.

- Kynganica, Bourg., Zanguébar.

- Letourneuxi, Bourg., Egypte.

- Zanzibarica, Bourg., Zanguébar.

\section{AURIGULIANA}

Limnaca expansilabris, Bourg., Égypte.

- Soleilleti, Bourg., Choa.

- Forskali, Letourneux, Égypte.

\section{AGUMINATIANA I}

Limnaea Gravieri, Bourg., Choa.

- de Baizei, Bourg., Zanguébar, Tanganika et Nyanza Oukeréwé.

\section{LIMOSIAYA}

Limmaea Laurenti, Bourg., Égypte, Tanganika.

1. L'Espèce type de ce groupe est la Limnxa acuminala de I'Indoustan. 
Limnara Alexandrina, Bourg., tout le bassin du Nil, Tanganika.

- Natalensis, Krauss, Natal.

- orophila, Morelet, Benguella.

- Bocageana, Morelet, Benguella.

- sordulenta, Morelet, Angola.

- Egyptiaca, Bourg., Égypte.

- Cleopatra, Letourneux, Égypte.

\section{AIIPULLACEANA}

Limnæea amygdalina, Bourg., Égypte.

\section{RAFFRAYANA}

Limnæa Raffrayi, Bourg., Égypte, Abyssinie.

- Lavigeriana, Bourg., Tanganika.

- Benguellensis, Morelet, Benguella.

- rthiopica, Bourg., Abyssinie.

- africana, Ruppell, Abyssinie, Zanguébar, Tanganika.

\section{WAHLIANA}

Limnæa astilba, Bourg., Égypte.

\section{PALLSTRISIANA}

Limnara Lessepsiana, Bourg., Égypte.

\section{TRUNGATLLIANA}

Limnea Iruncatula, Goupil(L. Umlasiana, Kuster), depuis l'Éggple jusqu'au Cap, dans presque tous les cours d'can de l'Afrique orientale. 
Comme on le voit, dès 1883, j’avais déjà signalé les noms des Limnées que je vais faire connaitre.

Ces Limnées, an nombre de six, dont trois inédites, proviemnent des cours d'eau du bassin du Vouami et dı Kyngani.

Limnea africana, Ruppell, in Bourguignat, Malac. Abyss., p. 95, fig. 99, 1883.

Échantillons bien semblables à ceux de l'Abyssinie dans le fleuve Kyngani.

Lamea ne Bazel, Bourguignat, Malac. Abyss., p. 89 (sans desc.), 1883, et Moll. Nyanza-Oukéréwé, in : Bull. Soc. malac. Fr., IV, 1S87, p. 268, et Iconogr. Tang., pl. 1, fig. 20, 1 SSS.

Fleuve Kyngani.

Linnea truxcatcla, Goupil, Moll. Sarthe, p 64, pl. II, fig. 1-3, 1835 (Buccinum truncatulum, Müller, 1774).

Cette Espèce cosmopolite a été constatée dans le cours du Vouami.

Limsea Caneroxi, Bourguignat, Malac. Abyss., p. 88 (sans desc.), 1883.

Celte Espèce, qui ressemble comme taille. comme coloration et comme aspect, à la Limnaca acroxa de la région nilotique', diffère néanmoins de cette Limnée par sa forme plus ovalaire, moins renflée; par sa spire moins grêle et non en disproportion de grosseur aussi prononcée que celle de l'acroxa; par son bord externe légèrement sinueux

1. Bourguiguat, Malac. abyss., p. 90, fig. 9't, 1883. 
supérieurement, puis inférieurement sensiblement convexe (celui de l'acroxa descend, d'une facon rectiligne, dans le sens de l'obliquité aperturale); enfin, chez la Limnxa Cameroni, les tours (au nombre de quatre) s'accroissent régulièrement en grosseur, bien que très rapidement en hauteur, tandis que chez la Limnxa acroxa, ils croissent chétivement jusqu'à la moitié de l'avant-dernier, puis prennent alors presque subitement un très grand développement. La spire de celte Limnée, par suite de ce développement disproportionné, paraîl si délicate, qu'clle semble anormale.

La Limnxa Cameroni parait abondante dans les cours d'eau de la valléc du Kyugani, à l'endroit du passage des caravanes.

Limnea Krygarica, Bolugulugnat, Malac. Abyss., p. 88 (sans desc.), 1883.

Cetle Limnée, également de la vallée du Kyngani, au sud de Bagamoyo, presque moitié plus petite que la Limnxa Cameroni, diffère notamment de celle-ci par son ouverture plus oblique, par son borl cxterne régulièrement convexe en avant, par sa columelle torse, presque lamelleuse et fortement arquée à sa partie inférienre. La base columellaire, par suite de l'obliquité aperturale, est sensiblement rétrocédente.

Limnea Zaxzibarica, Bourguighat, Malac.Abyss., p. 88 (sans dese.), 1883.

Pelite Espèce, d'une taille un peu plus faible (haut., 11 ; diam., 5 millim.) que la précédente, 
différant de celle-ci par sa forme moins ventrue, plus oblongue par suite de sa spire torse, plus grêle et plus régulièrement acuminée; par la direction très descendante de sa suture; par son axe columellaire moins tors et moins fortement arqué inférieurement; enfin, par sa base aperturale bien plus rétrocédente.

Cours d'ean de la vallée du Kyngani.

\section{PHYSOPSIS}

Les Espèces connues de ce genre africain sont au nombre de douze.

Physopsis africana, Krauss, Natal.

- abyssinica, Martens, Abyssinie, Choa.

- eximia, Bourguignat, Abyssinie.

- Stanleyana, Bourg., Zanguébar.

- preclara, Bourso, Zanguébar.

- globosa, Morelet, Angola.

- ovoidea, Bourg., Zanguébar.

- Letourneuxi, Bourg., Égypte.

- Lhotellerii, Bourg., Égypte.

- Soleilleti, Bourg., Choa.

- Meneliki, Soleillet, Choa.

- Leroyi, Grandidier, Onsaghara.

Sur ces douze Espèces, que j'ai fait connaitre en $1879^{1}$ et en $1885^{2}$, les suivantes ont été recueillies dans le champ malacologique de ce travail.

1. Moll. Égypte, Abyssinie, Zanzibar, etc., p. 12 et suiv.

2. Moll. dn Chor, p. 25 et 26 . 
Physopsis Stanleyaxa, Bourguigual, Mollusques Égypte, Abyss., Zanzib., ete., p. 14, 1879.

Cours d'eau dans la basse vallée du Kyngani, non loin de Bagamoyo.

Pirysopsis preclara, Bourguignat (loc. sup. cit.), p. 14.

Fleuve Kyngani.

Physopsis ovordea, Bourguignat (loc. sup. cit.), p. 16.

Cours dı Kyngani, près Bagamoyo, et cours d'eau aux environs de Kondoa, dans la haute vallée du Vouami.

Piysopsis Soleilleti, Bourguignat, Moll. Choa, p. 25, pl. I, fig. 11, 1885 .

Cette Espèce choane a été retrouvée dans le cours de l'Ouébi, près de Guélidi, par le voyageur G. Revoil.

Pursopsis Lenoy, Grandidier, Moll. Ousaghara, etc., in : Bull. Soc. malac. Fr. IV, p. 189, 1887.

Cours d'eau aux environs de la maison des RR. PP. du Saint-Esprit, dans l'Ousaghara.

A ces Espèces décrites depuis quelques années, j'ai à ajouter la nouvelle forme qui suit.

PHISOPSIS BLOYETI, spec, nov.

Testa oblongo-ovoidea, superne obtusa, inferne attenuata, solidula, opacula, cornea, polita aut sub. tilissime substriatula; - spira brevi, obtusa (apex minutus); - anfr. 5 oblongo-convexis, suturaim- 
pressula separatis; - ultimo magno, $3 / 4$ altitudinis aquante, regulariter convexo, inferne prope columellam angulo cervicali carinam simulante (angulus usque ad basin apertura descendens) notato; - apertura subobliqua, elongato-angusta, externe exacte convexa, superne angulata, inferne coarctata ac valide canaliculata; - columella recta, lamellosa (lamella prominens, contorta, usque ad basin prolongata); - margine externo acuto, intus leviter labiato; - callo palatali nullo. - Alt., 12; diam., 6 ; alt. ap., 9 ; lat., 3 millim.

Cette Espèce ne peut être rapprochée que de la Physopsis oroidea; elle se distingue de celle-ci par sa taille moindre, par son test plus poli et plus délicat, par sa suture non linéaire, par son ouverture plus étroite et dont la base est plus fortement canaliculée, par sa lamelle columellaire plus saillante, par son manque de callosité palatale, mais surtout par l'arête cervicale qui longe sa columelle.

Cette Physopside, dédiée au brave commandant Bloyet, vit dans les cours d'eau des environs de Kondoa, dans l'Ousaghara. 


\section{X}

\section{PALUDINID A}

Les Espèces de cette famille appartiennent aux genre Vivipara et Cleopatra; il n'est pas à ma connaissance que i'on ait recueilli, dans les régions équatoriales, des Bythinies, des Amnicoles, des Paludestrines ou des Paludinelles, bien que je sois moralement certain que de nombreux représentants de ces genres doivent exister dans les cour's d'eau de ces pays.

\section{VIVIPARA}

Je ne connais que trois formes vivipariennes, formes qui toutes les trois ont été primitivement découvertes dans les eaux du lac Nyassa el dans celles du Shiré et du Zambèse.

Vivipara caplllata, Frallenfeld, Zool. mise, in : Verh. zool. Gesellsch. Wien, 1865, p. 533, pl. xxı, et Grandidier, in : Bull. Soc. malac. Fr., IV, 1887, p. 190.

Échantillons bien semblables à ceux dı lac Nyassa. Espèce abondante dans le Vouami et le Kyngani.

Vivipara Robertsoni, Frauenfeld (loc. sup. cit.), p. 533, pl. xxı, et Grandidier, in : Bull. Soc. malac. Fr., IV, 1887, p. 190. 
Cours d'eau près du couvent des RR. PP. du Saint-Esprit.

Vivipara JefFreysi, Frallenfeld (loc. sup. cit.) p. $532, \mathrm{pl}$. xxil.

Cours d'eau de l'Ousaghara, notamment aux environs de Kondoa.

\section{CLEOPATRA}

Les Cleopatras connues de ces régions peuvent se répartir :

$1^{\circ}$ En Espèces de forme allongée-pyramidale, à sommet acuminé et à perforation ombilicale nulle ou à peine accusée. (Cl. Letourneuxi, Cameroni, Kynganica, africana, exarata, Zanguebarica Panli et Ajanensis.)

$2^{\circ}$ En Espèces non allongées-pyramidales, mais de forme écourtée-globuleuse, à perforation ouverte, et ayant de certains airs de ressemblance avec de petites vivipares. (CI. Guillemeti ${ }^{1}$.)

Cheopatha Letounneuxi, Bourguighat, Moll. Égypte, Abyss., Zanzib., etc., p. 19, 1879.

Cours d'eau de la vallée du Kyngani, au sud de Bagamoyo; le Vouami, au-dessus de Sadani; le Makata et le M'kondokoua, au sud et à l'est de la station de Kondoa, dans l'Ousaghara.

Cleopatra Cameroni, Bourguignat (loc. sup. cit.), p. 21, 1879, et Grandidier, Moll. Ousagh. in : Bull. Soc. malac. Fr. IV, 1887, p. 191.

1. La Cleopatra Jouberti (Bourguignat, Iconogr, malac tang., pl. w, fig. 3, 1888) appartient à ce groupe. 
Le Kyngani, au sud de Bagamoyo, et les cours d'eau dans la haute vallée de ce fleuve, sur le territoire des Oukami, ainsi que dans les rivières ousaghariennes de la partie hante de la vallée du Vouami.

Cleopatra Kynganica, Bourguighat (loc. sup. cit. ), p. 21, 1879.

Le Kyngani, dans sa partie inférieure, voisine de Bagamoyo.

Cleopatina africana, Bourguignat, Moll. Choa, p. 29, 1885 (Paludomus africanus, Martens, Hildebr. conch. in : Monatsb. k. ak. Wiss. Berlin, 1878, p. 297, pl. II, fig. 11-13).

Cette Espèce, signalée à Finboni, sur la côte de Zanzibar, vit également dans le Vouami, au-dessus de Sadani.

Cleopatra exarata, Bourguignat, Moll. Choa p. 29, 1885 (Paludomus exaratus, Martens, Hildeb. conch. in : [loc. sup. cit.], p. 297, pl. II ', fig. 14-16, 1878.)

Cette belle Espèce, également découverte à Finboni, a élé retrouvée en abondance dans le Mlédyi, près de Ribé, petite rivière qui se jette dans l'Océan indien, proche Monbas, ainsi que dins la rivière de Pagani, entre Tanga et Sadani.

Cleopatra Zanguebarica, Bourguignat, in Sched. 1857 (Melania Zanguebarensis, Petit, in : Journ. Conch., II, 1851, p. 263, pl. vir, fig. 1).

1. Sur celte planche, le nom d'exaralıs a été modifié en celui de cingulata. 
Cette coquille zanzibarienne parait commune dans les cours d'eau de la vallée du Vouami.

Cleopatra Pauli, Bourguignat, Moll. Choa, p. 27, fig. 3, 1885.

Celte Espèce du fleuve Haouach, dans le Choa, a été retrouvée bien identique dans la rivière de Magogo (Ougogo).

Cheopatra annensis, Bourguignat, Moll. Choa, p. 29, 1885 (Paludomus Ajanensis, Morelet, Sér. conch., II, 1860, p. 110, pl. vi, fig. 10).

Rivière de l'Ouébi, près Guélidi.

Cleopatra Gullemeti, Bourguignat, Esp. nouv. et genres nouv. Oukér, etc., p. 6, 1885.

Petit ruisseau de Sagati, affluent du Guéringuéré, dans l'Oukouéré. 


\section{XXI}

\section{AMPULLARID E}

Les Ampullaridées des régions équatoriales appartiennent aux genres Ampullaria, Meladomus et Leroya .

\section{AMPULLARIA}

J'ai, d'abord en 1863 ', donné une première liste des Ampullaires africaines; puis, en 1879?, une seconde qui portait à douze les formes connues à cette époque. Depuis, notre ami, M. René Billotte, dans son travail monographique des Espèces de ce genre ${ }^{3}$, a porté le chiffre à dix-neuf.

Voici maintenant les Espèces connues du continent.

Ampularia adusta, Reeve, Conch. iconogr., fig. 11 et Marlens, Moll. Deck., p. 60, 1869. - Zanzibar.

Ampulamia balenoidga, Gould, in : Journ. conch., II, 1851, p. 267, pl. vr, fig. 8. - Cap des Palmes sur la côte ouest de l'Afrique.

Ampullaria Bourgulgnati, Billolle, in : Bull. Soc. malac. Fr., 1885, p. 107, pl. vr, fig. 3.-Egypte.

Ampullaria Bridouxi, Bourguignal, Iconogr. malac. Tang., pl. v, fig. 22, 1888. - Tanganika.

1. In : Moll. nouv. (3e décade), p. 78.

2. Moll Égyple, Abyss,, Zanz., etc., p. 32.

3. In : Bull. Soc. malac. Fr., 1885, p. 1 
Ampullaria Cinamesiana, Billotle, in: Buti. Soc. malac. Fr., 1885, p. 106. - Nil, au-dessus de Gondokioro.

Ampellaria Demesnilaxa, Billotte (loc.sup.cit.), p. 104, pl. vi, fig. 2 - Vallée de l'Ouébi-Doboi (Comalis), et ì Ousagozi Ounyamouezi) entre Tabora et Oudjiji.

A npllaria ervistirostoma, Reeve, Iconogr. Mon. Amp. $n^{\circ} 59$, pl. xı1. - Zanzibar.

Anpulanis exigua, Philippi, Mon. Amp.,p. 46, pl. xıı, fig. 4. - Egypte.

Anplluira gradata, Smith, in : Proc.zool. Soc. Lond., 1881, p.289, pl. xxxı1, fig.22. - Lae Nyassa et Ousaghara.

Ampleluna hordorana, Parreyss, in : Philippi, Mon. Amp., p. 44, pl. xur, fig. 1, 1851, et Bourguignat, Moll. nouv. (3éc. 1863), p. 78, pl. xi, fig. 12-13. (Amp. ovata [non Olivier!], Savigny, pl. expéd.Égypte,Coq., pl.ı, fig. 25, et Cailland, Voy. Méroë, Atlas II, pl. Lx, fig. 10, 1823, et t. IV, p.264, 1827, etc.) - Égypte, Nubie, Abyssinie.

Impullaria Largillete, Philippi, in : Zeitschr. f. Malak., p. 192, 1848, et Mon. Amp., 1851, p. 46, pl. xır, fig. 5. - Côtes de Mozambique.

Ampelaria Letolreuxi, Bourguignat, Moll. Égypte, Mbyss., Zanz., p. 29, 1879. - Zanguébar.

Ampullaria lucida, Parreyss, in: Philippi, Mon. Amp., p. 45, pl. xıı, fig. 2, 1851. - Éggpte.

Amplelaria occinevtalis, Moussoll, in : Journ. 
conch., 1887, p. 299, pl. xu, fig. 9. - Benguéla.

Ampullaria onata, Olivier, Voy. Emp. ottom., II, p. 39, Atlas, pl. xxxi, fig. 1, 1804, et Philippi, Mon. Amp., 1851, p. 49, pl. xrv, fig. 5, et Bourguignat, Moll. nouv. (3e déc.), 1863, p. 79, pl. x, fig. 11.Egypte. Tanganika.

Ampuldara Raynond, Bourguignat, Moll. nouv. (3 déc. 1863), p. 76, pl. ıx, fig. 4. - Égypte.

Ampularia Revoli, Billote, in: Bull. Soc. mal. France, 1885, p. 103. - Comalis.

Ampullari Rucietiana, Billotle(loc. sup. cit.), p. 105, pl. vi, fig. 1. - Comalis.

Ampulcana specrosa, Philippi, in: Zeitschr. f. Malak., p. 18, 1849, et Mon. Amp., p. 40, pl. xi, fig. 2, 1851. - Comalis.

Ampullaria vitrea, Reeve, lconogr. Mon. Amp., pl. xvir, sp. 80 (Helix vitrea, Born, Test. mus. Vindob. pl. xv, fig. 15-16). - Égypte.

Ampuldari Welwitscin, Bollguignat, Moll. Égypte, Mbyss., Zanz., p. 31 et 32, 1879 ( Imp. ovata [non Olivier!] Morelet, Moll. Welw., p. 94, pl. 1x, fig. 10, 1868. - Le Niger.

Ampularia Merner, Philippi, Mon. Amp.,p. 19. pl. xvir, fig. 2, 1851 (non Amp. Wernei de la pl. v, f. 4, qui est une variété minor de l'Amp. Charmesirna).-- Nil Blanc. Albert-Nyanza.

Sur ces vingt-deux Espèces, six seulement ont été constatées dans ces régions équatoriales, savoir : 
Ampuldaria Dumesniliana, dans la rivière de l'Ouébi, entre Meurka et Moguedonchou; ainsi qu'à Ousagozi, dans l'Ounyamouezi ;

Amellaria gradita, dans le M'kondokona et le Makata, affluent du Vouami dans l'Ousaghara ;

Anpelania Letourieuxi, dans le Kingani, près de Bagamoyo, à l'endroit du passage des caravanes ;

Ampulara Revolu, dans les marais et les cours d'eau entre Meurka et Mognedouchou.

Ampllaria Ruchetuxa, dans l'Ouébi, all-dessus de Guélidi.

Ampuldaria speciosa, dans l'Ouébi, également au-dessus de Guelidi.

\section{MELADOMUS !}

Sous ce nom générique, je comprends toutes les Ampullaridées Sénestres, à l'exception des Leroyes, que les auteurs ont tantôt confondues avec les vraies Ampullaires ${ }^{2}$, ou tantôt classées sous le nom de Lanistes, ou enfin séparées, à l'exemple des frères Adams ${ }^{3}$, en Meladomus et en Lanistes.

Le nom de Meladomus a été proposé, en 1840, par Swainson, pour la Paludina olivacea de Mozambique; celui de Lanistes, en 1810, par Denys de

1. Swainson, Treat. mal., p. 340, 1810 (Lanistes Denys de Monlforl, Conch. syst., 1I, p. 122, 1810, non Lanistes, Humphrey, Mus. Calonn., 1797).

2. Les Ampullaires sont toutes dextres.

3. Genera of shells, I, p. 3i8-49.

4. Ne pas confondre avec celui de Paludomus. 
Nontfort ${ }^{1}$, pour le C'yclostoma carinata d'Égypte. Ces deux Espèces, olivacea et carinata, appartiennent au genre Meladomus.

Je n'ai pu adopter l'appellation de Lanistes, bien que plus ancienne, parce qu'il existe dans le $M / u-$ seum Calonnianum d'Humphrey, publié en 1797, un genre établi, sous le mềne nom, pourle Mytilus discors de Linnieus.

En 1879?, j’ai donné un recensement des Espèces de ce genre essentiellement africain. Ces Espèces étaient alors au nombre de vingt; actuellement, en y comprenant celles que je vais décrire, elles atteignent un chiffre plus considérable; elles appartiennent aux cinq séries suivantes:

$1^{\circ}$ Purpuriaxa. Coq. aliongée, plus ou moins conoüde, à test poli, à tour's peu bombés et à suture peu profonde.

Meladomus purpuneus, Bourg., Moll. Égypte, Abyss., Zanz., etc., p. 35, 1879 ( Ampullaria purpurea, Jonas, in : Arch. f. naturg., p. 242, pl. x, fig. 1, 1839, et Philippi, Mon. Amp., p. 22, pl.vi, fig. 1, 1851 ; - Bulimus tristis, Jay, Cat. Shells, p. 121, pl. vil, fig. 1, 1839; - Meladomus olivaceus [non Sowerby], H. et A.Adams, Gen. Shells [5 livr.1854], p. 349, pl. xxxv1, fig. 6 ; - Lanistes purpureus, Dohrn, Moll. Zamb. in : Proc. zool. Soc. Lond., 1865, P. 233; et Pfeiffer, Novit. conch., p. 293, 1866 ; et

1. Conclı, syst., II, p. 122.

2. Bourguignat, Moll. Égypte, Abyss., Zanz., rle, p. 33 et suir. 
Smill in Proc. zool. Soc. Lond., 1881, p. 290). Mozambique ${ }^{1}$ et Zanguébar, dans l'ile Zanzibar et les cours d'eau de la vallée du Kyngani.

Meladonus Bloyeti, Bourguignat, spec. nov. Grande Espèce (haut., 65; diam., 40; haut.ouv., 34; lar. ouv., 22 millim.) turriculée, conique, solide, d'une teinte foncée marron-olivâtre; fente ombilicale profonde et étroite; tours convexes (les supérieurs relativement exigus), à croissance régulière, à suture peu profonde; ouverture suboblique d'une forme ovalaire fortement anguleuse supérieurement; péristome droit, aigu, faiblement réfléchi du còté columellaire, qui est presque rectiligne.

Celte Espèce, dédiée au commandant Bloyet, se distingue du Melad. purpureus par sa spire conique à sommet aigu, par sa forme moins ventrue, plus élancée, par ses tours plus délicats, moins gros, par son ouverture moins large, etc.

Cours d'eau aux environs de Kondoa (Ousaghara).

Meladonus pyramidis, Letourneur in : Bourguignat, Noll. Egypte, Abyss., Zanz., p. 36, 1879. - Fleuve Kyngani, près de Bagamoyo, et rivière de Nyahoua, dans l'Oukimbou.

Meladonus mitidissimus, Bourguignat, spec. nov. - Coq. d'assez grande taille (hant., 40 ; diam., 26 ; haut. ouv., 21 ; larg., 14 millim.), brièvement conique, à sommet nćanmoins un peu obtus. Test mince, très brillant, olivàtre, poli, lisse, sauf vers

1. Telle sur le Zambèse; lagune près Carabaçeira. 
l'ouverture. Six tours peu convexes, à croissance régulière et à suture presque superficielle. Ouverture presque verticale, échancrée, oblongue, supérieurement anguleuse. Columelle réfléchie sur une petite fente ombilicale. Péristome mince et droit.

Cours d'eau sur la rive gauche du Kyngani, près de Bagamoyo, et rivières de la haute vallée du Vouami, aux environs de Kondoa (Ousaghara), ainsi que dans la plaine marécageuse de l'Ouha, sur la rive du Roussoughi.

$2^{\circ}$ Olivaceaxa ${ }^{2}$. Coq. à spire très torse, à tour's très convexes, à suture profonde.

Meladonus olvaceus, Swainson, Treat. malac., p. 340, 1840 (Paludina olivacea, Soserby, Gen. shells, Palud., fig. 3, et Lov Reeve, Conch. syst., II, p. 197, fig. 3; Lanistes olivaceus, Pfeiffer, Novit. conch., p. 291, 1866). - Mozambique.

Meladonus procerus, Bourguignat, Mollusques Égypte, Abyss., Zanz., etc., p. 34, 1879 (Lanistes olivaceus, var. procerus, Pfeiffer, Novit. conch., p. 292, pl. Lxxi, fig. 1-2, 1866). - Cours d'eau du Mozambique et du Zanguébar, dans les rivières au nord de Dar-ès-Salam.

Meladonus ambigues, Bourguignat, Mollusques Égyple, Abyss., Zanz., p. 34, 1879 (Ampullaria

1. A ce groupe apparticut encose le Veladomus Grasscti de Madagascar (Bourguignal, Moll. Égyple, Abyss., efc., p. 34, 1879 [Ampullaria Grisseti, Morclel, in : Jouriu. concls., p. 267, pl. x, fig. 1-2, 1863]). 
ovum [non ovum de Pelers], Philippi, Monogr. Amp. [exclud. desc.], pl. vir, fig. 7, 1851; Lanistes olivaceus, var. ambigua, Pfeiffer, Novit. conch., p. 292 [avril 1866], pl. Lxxi, fig. 3-4). - Mozambique.

Meladonus elupticus, Bourguignat, Mollusques Égypte, Abyss., Zanz., p. 35, 1879 (Ampullaria elliptica, Martens, mss.; Lanistes ellipticus, Pfeiffer, Novit. conch., p. 294 [avril 1866], pl. Lxx, fig. 9-10; Lanistes Zambesianus, Furlado, in : Journ. conch., pl. VII, fig. 1, 1886). - Mozambique.

Meladomus Pfeiffeni, Bourguignat, Mollusques Égypte, Abyss., Zanz., p. 37, 1879 (Lanistes Libycus ${ }^{1}$, var. Pfeiffer, Novit. conch, p. 286, pl. Lxx, fig. 5-6, 1866). - Cours d'eau de la haute Guinée.

Meladomes elation, Bourguignat, Moll. Égypte, Abyss., Zanz., p. 35, 1879 (Lanistes ovum, var. elatior, Pfeiffer, Novit. conch., p. 291 [avril 1866], pl. Lxx, fig. 7-8).

Rivières près de Monbas, ainsi que dans le cours d'eau près de Tanga (côte du Zanguébar). Cette Espèce est également connue du fleuve Niebolır (Mozambique).

Meladomus ovum, Bourguighat, Moll. Égypte, Abyss., Zanz., p. 36, 1879 (Ampullaria ovum, Peter's, in : Arch.f. naturg., I, 1835, p. 315; et Philippi, Monogr. Amp., p. 22, pl. vr, fig. 2, 1851 ;

1. Non Ampullaria libyca, de Morelet. 
Lanistes ovum, Dohrn, Moll. Zamb., in : Proc. zool. Soc. Lond., 1865, p. 233; et Pfeiffer, Novit. conch., p. 290, 1866; et Smith, in : Proc. zool. Soc. Lond., 1877, p. 715). - Rivières et fleuve de la côte de Mozambique. Tette sur le Zambèse. Lac Nyassa.

Dans le Zanguébar, cette Espèce a été reconnue dans les cours d'eau du Kyngani, du Rufidgi, au nord de Quiloa, et dans ceux des environs de Darès-Salam, ainsi que dans l'ile Zanzibar.

Meladomus jouberti, Bourguignat, Iconogr. Anim. Moll. Tang., pl. vi, fig. 6, 1888.

Lac Tanganika, à l'embouchure du Nalagarazi, et dans le Rousoughi, afluent dextre du Malagarazi, sur le territoire des Ouha, entre Tabora et Oudjiji.

Meladomus sinistronsus, Bourguignat, Moll. Égypte, Abyss., Zanz., p. 39, 1879 (Paludina sinistrorsa, Lea, in : Trans. amer. Phil. Soc., VI, et Observ. g. Unio, p. 90, pl. xxı1, fig. 78; Ampullaria sinistrorsa, Philippi, Monogr. Amp., p. 64, pl. xxi, fig. 3, 1851). - Le Malagarazi et dans un grand nombre de cours d'eau de l'Ounyamouezi et de l'Ousaghara.

Meladonus intontus, Bourguignat, Moll. Égypte, Abyss., Zanz., p. 39, 1879 (Ampullaria intorta, Lamarck, Anim. s. vert., VI, $2^{\circ}$ part., p. 179, 1822, et [édit. Desh.] VIII, p. 541, 1838; et Philippi, Monogr. Lmp., p. 23, pl. v1, fig. 3, 1851. Loc. inconnue. 
Meladomus guinaicus, Bonrguignat, Mollusques Égypte, Abyss., Zanz., p. 39, 1879 (Cochlea guinaiea, Chemnitz, Coneh. cab., IX, 1786, p. 80, fig. 913-14; Ampullaria Guinaiea, Lancarck, Anim. s. vert., VI, $2^{\mathrm{e}}$ partie, 1822, p. 178, et [édit. Desh] VIII, 1838, p. 536, et Philippi, Mon. Amp., p. 28, pl. vu, fig. 6, 1851; Lanistes Guinaicus, Pfeiffer, Novit. conch., p. 289, 1866. - Guinće.

Meladonus arfixis, Bourguignat (Lanistes affinis, Smith, in : Proc. zool. Soc. Lond., 1877, p. 716, pl. Lxxiv, fig. 7, et 1881 , p. 290, pl. xxxiv, fig. 23). - Lac Nyassa (Mozambique), et dans la vallée entre le pays de Kiti et celui d'Ougula (Zanguébar).

Meladones solode, Bourguignat, in sched., 1884, et Grandidier, Moll. Ousagh., in: Bull. Soe. malae. Fr., IV, 1887, p. 191 (Lanistes solidus, Smith, Sh. Nyassa, in : Proc. zool. Soc. Lond., 1877, p. 716 , pl. Lxxıy, fig. 10-11). - Lae Nyassa ou se tronve le type. Cours d'eau de la haute vallée du Vouami, dans I'Ousaghara; le Kyngani, sur le territoire des Oukami.

Meladonus Deguerrinus, Bourguiguat, spec. nov. - Coq. de petite taille (haut., 22 ; diam., 21 ; haut. ouv.. 14; larg., 10 millim.), très torse, fragile, olivàtre, polie, pourvue d'une perforation étroite très profonde. Spire très obtuse, à sommet rond. Cinq fours obtus-convexes (les supérieurs très gros), à croissance rapide, à suture relativement peu profonde. Ouverture oblique, ovalaire. 
Columelle réfléchie, subrectiligne. Péristome non continu, mince, droit. Callosité à peine sensible.

Cette Espèce, dédiée au R. P. de Guerry, supérieur général des Missionnaires d'Afrique, a été recueillie dans le Kyngani, près de Mbiki (Oukouéré) et de Makata, afluent supérieur du Vouami, dans l'Ousaghara.

$3^{\circ}$ Libreinna. Coq.torse, globuleuse, à spire peu élevée, à perforation entourée par une angulosité.

Meladomus libycus, Bourguignat, Moll. Égypte, Abyss., Zanz., p. 38, 1879 (Ampullaria libyca, Morelet, in : Rev. zool., p. 364, 1848, et Philippi, Monogr. Amp., p. 25, pl. vi, fig. 8, 1851, et Morelet, Sér. conch., I, 1858, p. 28, pl. in, fig. 9). - Cours d'eau du Gabon.

Meladonus niloticus, Bourguighat, Mollusques Egypte, Aljyss., Zanz., p. 40, 1879 (Ampullaria nilotica, Sowerby, in : Swainson, Zool. illust., $2^{\mathrm{e}}$ série, I, pl. xxxvin, fig. 2, 1831, et Plillippi, Monogr. Amp., p. 24, pl. vi, fig. 6, 1851). - Cette Espèce, improprement nommée nilotica, dont la patrie est inconnue, doit provenir du Gabon.

Meladonus holostona, Bourguignat, Moll. Égypte, Abyss., Zanz., p. 37, 1879 (Ampullaria holostoma, Morelet. in : Journ. conch., VIII, 1860, p. 191). - Haute Guinće.

Meladomus Benxardaxus, Bourguignat, Moll. Égypte, Abyss., Zanz., p. 38, 1879 (Ampullaria Bernardiana, Morelet, in : Journ., conch., VIII, 
1860, p. 190 [non Lanistes Bernardianus de Pfeiffer, qui est une Espèce différente à rapporter au Meladomus Vignoni]). - Haute Guinée.

Melanonus Vignoxi, Bourguighat, spec. nov. (Lanistes Bernardianus [non Ampullaria Bernardiana de Morelet], Pfeiffer', Novit. conch., p. 286, pl. Lxx, fig. 1-4, 1866). - Haute Guinće.

Meladomus subcarixatus, Bourguighlat, Moll. Égypte, Abyss., Zanz., p. 40, 1879 (Ampullaria subearinata, Sowerby, Gen. shells, fig. 4, et Philippi, Monogr. Amp., p. 24, pl. vi, fig. 7, 1851). Congo, Angola et Benguéla.

Mleladonus cilatus, Bourguignal, in sehed., 1887. (Lanistes ciliatus, Martens, Hildebr. conch., in : Monatsb. k. akad. Wiss. Berlin, 1878, p. 296, pl. II, fig. 8-10.)

Espèce globuleuse, de petite taille (haut., 17-18; diam., 16.17 millim.), pourvue d'une perforation circonscrite par une arête anguleuse, remarquable, en outre, par son test entouré de sillons spiraux pilifères et par ses tours (sauf le dernier) plans et anguleux autour de la suture. - Finboni, sur la côte de Zanzibar.

D'après la figure 8 donnée par le $D^{r}$ Martens, cette coquille, dont l'auteur, dans sa diagnose, n'indique pas l'épaisseur du test, parait si épaisse que l'on se demande si elle appartient bien au genre où elle est placée.

Meladonus Alexaxdr, Bourguiguat, spec. nov. Cette Espèce, à laquelle j'attribue l’e prénom du 
R. P. missionnaire Alexandre Le Roy, ressemble comme forme au Méladome précédent, dont elle diffère, néanmoins, par son test transparent, délicat, par le manque complet de sillons spiraux pilifères, et par un méplan circumsutural plus prononcé et circonscrit par une angulosité imitant une carène aigue.

Ce Meladomus Alexandri, qui est abondant en amont de Sadani, dans les affuents du Vouami, est une coquille globuleuse, de taille médioere (haut., 16-17; diam., 14-15; haut. ouv., 11; larg., 7 millim.), à test mince, fragile, transparent, très brillant, presque poli ou très finement striolé, pourvu d'une étroite perforation, circonscrite par une angulosité. Ses tours, au nombre de cinq, sont vigoureusement carénés et bien plans autour de la suture, jusqu'au dernier tour qui est régulièrement convexe. Son ouverture verticale, très échancrée, semi-ovale, anguleuse ì la base columellaire, est entourée par un bord péristomal non patulescent, rectiligne et d'une grande fragilité.

$4^{\circ}$ Bolteniana. Coquille également torse, mais moins globuleuse, à spire érrasée, à ombilic bien ouvert, entouré d'un angle prononcé (sauf chez le Letourneu.xi).

Meladonus Boltenunus, Bourguignat, Moll. Égypte, Abyss., etc., p. 41, 1879 (Helix Bolteniana, Chemnitz, Conch. cab., IX. 1, p. 89, pl. cix, fig. 92L-22, 1786. - Cyclostoma carinata, Olivier, Voy. Emp. ottom., II, p. 39 (en note), et Atlas, 
pl. xxxı, fig. 2, 1804; - Lanistes Olivieri, Denys de Montfort', Conch. syst., p. 122, 1810, et Blainville, Man. malac., pl. xxxiv, fig. 3, 1825; - Ampullaria carinata, Lamarck, Anim. s. vert., VI, 2, p. 179, 1822, et Caillaud, Voy. Méroé, II, 1823, et Atlas, 1827. - Ampullaria Bolteniana, Philippi, Mon. Amp., p: 23, pl. vi, fig. 4-5, 1851, et Bourguignat, Moll. nouv. ( $3^{\text {e }}$ déc. 1863), p. 80; - Ampullaria xegyptiaca, Ehrenberg, teste Jickeli, Moll. n. o. Afri., 1874, ete.).

Espèce abondante dans les cours d'eau du bassin du Nil, mais particulièrement dans les canaux de la basse Égypte. - Ce Méladome vit également dans l'Ouébi, près de Gućlidi, où il a été recueilli par le voyageur G. Revoil. Les échantillons de cette localité sont identiques à ceux du Nil.

Meladonus Duveyraerianus, Revoil, in : Bull. Soc. malac. Fr., II, 1885, p. 99, pl. vi, fig. 5.

L'Ouébi entre Meurka et Moguedouchou.

Meladonus Letourneuxi, Bourguignat, Moll. Égypte, Abyss., Zanz., ete., p. 41, 1879, et Berthier, in : Bull. Soc. malac. Fr., II, 1885, p. 101, pl. vi, fig. 4.

Basse Égypte.

$5^{\circ}$ Nyassaxa. Coquille de forme subtriangulaive à spire presque plane.

Melanonus Nyassanus, Bollguignat, Moll. Égypte, Abyss., Zanz., ete., p. 39, 1879. (Lanistes

1. C'est pour cette Espèce que le gence Lanistes a été créé. 
Nyassanus, Dohrn, Moll. Zamb., in : Proc. zool. Soc. Lond., 1865, p. 233, et Smith, in : Proc. zool. Soc. Lond., 1877, p. 715.)

Lac Nyassa et lac Pamolombue (Zambèse).

Tels sont les Méladomes connus du continent. Sur ces Espèces, seize (Purpureus, Bloyeti, pyramidalis, nitidissimus, procerus, elatior, ovum, Jouberti, sinistrorsus, affinis, solidus, Deguerryanus, ciliatus, Alexandri, Boltenianus et Duveyricrianus), dont plusieurs nouvelles, ont été constatées dans les régions équatoriales qui m'occupent en ce moment.

\section{LEROYA}

Je renvoie à l'excellent travail de M. Alfred Grandidier, sur les Mollusques de l'Ousaghara ', pour la connaissance des caractères de ce singulier genre.

Les deux Leroya connues sont :

Leroy Bourgugrati, Grandidier, Moll. Ousaghara, in : Bull. Soc. malac. Fr., IV, 1887, p. 192, et Bourguignat, Iconogr. malac. Tang., pl. vi, fig. 2-5, 1888.

Cours du Vouami, ainsi que dans le Malagarazi et le lac Tanganika.

Lenoya chanmetanti, Grandidier (loc. sup. cit.), p. 193, 1887.

1. In : Bull. Soc. malac. Fr , IV, 1887, p. 185 et snivantes. 
Cette Espèce, dont je donne la représentation sur la planche nu (fig. 21-22), est une forme moins ventrue, plus allongée, plus acuminée que la précédente; elle provient également du Vouami. 


\section{XXII}

\section{MELANID A}

Les Mélanidées, qui possèdent de si nombreuses Espèces dans les lacs Nyassa et Tanganika, sont pauvrement représentées dans les cours d'eau des régions équatoriales. Je n'en connais que cinq Espèces appartenant aux genres Melania, Tiara et Plotia.

\section{MELANIA}

Les vraies Mélanies, telles que Lamarck les a comprises, sont des Espèces caractérisées par un test allongé, turriculé, acuminé, à tours nombreux sans spinules, seulement cerclés de sillons spiraux avec quelques indices de nodosités, et pourvues d'une ouverture entière, ovalaire, à columelle légèrement arquée et à bord externe aminci.

Melaxia tuberculata, Bourguignat, Moll. orient., p. 65, 1853, et Malac. Algérie, II, 1864, p. 251, pl. $\mathrm{xr}$, fig. 1-11, el Iconogr. malac. Tang., pl. xı, fig. 26-27, 1888. (Nerita tuberculata, Müller, 1774; Melania fasciolata, Lamarck, 1822.)

Les échantillons de celle Espèce cosmopolite sont bien caractérisés; ils sont surtout abondants dans le Voé 1, l'Ouébi, le Vouami et le bas Kyngani.

1. Ou Nangando à Kilifi, entre Monbas et Mélinde. 
Melania Inimabanica, Martens, Peters Mossamu. Moll., in : Malak. Bl., 1859, p. 216, pl. II, fig. 10.

Celte belle Mélanie, découverte dans la vallée du Zambèze, se trouve bien Iypique dans le cours inférieur du Vouami. Les échantillons de cette localité ne sont pas tronqués comme ceux de Mozambique, mais bien entiers; ils ont une spire effilée (composée de 11 à 12 tours) ressemblant beaucoup, comme mode de costulations, à celle de l'Espèce précédente.

\section{TIARA}

Cette coupe générique, établie par Bolten ęt adoptée par M. Brot (Melaniaceen, p. 288, 1874), se compose de fort jolies Espèces ornées d'une rangée de spinules saillantes à la partie supérieure des tours.

Je crois qu'il convient de rapporter à cette coupe celle des Tiaropsis, créce par M. Brot (Melan., p. 299), parce qu'à mon sens les signes invoqués par l'auteur ne me paraissent pas différer suffisamment de ceux des Tiara.

Tiara crexularis, $H$. et $\Lambda$. Adams, Genera of Moll., I, p. 295, 1854. (Melania crenularis, Deshayes, in : Guérin, Mag. zool., pl. Lxxxu1, 1844.)

Varietas B. Tiara Vouamica, Bourguignat (Melania cremularis, Martens, Peter's Moss. Moll., in : Malak. Bl., 1859, p. 216 , et Ueber's. Moss. gesamm. Moll., p. 733, 1879).

Ce n'est pas le type de la crenularis des iles 
Philippines, mais une forme particulière de cette Espèce qui a été recueillie dans le fleuve Vouami, au-dessus de Sadani. Cette forme diffère du type par une taille moindre ${ }^{1}$, par un test d'une teinte olivâtre uniforme ${ }^{2}$, par des spinules un peu moins écartées, enfin, par une ouverture sensiblement plus étroite à la base.

Les échantillons du fleuve Vouami ne sont pas tronqués. La spire, composée de 8 à 9 tours, est bien entière.

Je rapporte à cette variété, que je distingue sous le nom de Tiara l'ouamica, la forme que le $\mathrm{D}^{\mathrm{r}}$ Martens indique de Mozambique sous l'appellation de Melania crenularis.

\section{PLOTIA}

Le genre Plotia, également élabli par Bolten (1798) et adopté par les frères $H$. et $A$. Adams ${ }^{3}$ et par G. Nevill ${ }^{4}$ comme coupe sous-générique du genre Tiara, a pour type la Melania spinulosa (Lamarck) des iles des Indes orientales.

Les Espèces de ce genre, très répandues dans toutes les iles de l'Océan indien, ainsi que dans le sud de l'Indoustan et de l'Indo-Chine, sont des formes mélaniemnes à spire acuminée, fort peu

1. La taille de celte variété est: haut, 27; diam., 14; haut. ouv., 15; larg., 7 mill.

2. Celui de la crenularis (type) est d'un noir bleuâtre très fencé.

3. Genera Moll., I, p. 295, 1854.

4. Hand list Moll, ind. Mus. Calc., 11, p. 280, 1884. 
scalariforme et plus allongée que celle des Tiara, i test assez mince, ì tours ornés, à leur parlie supérieure, de grosses côtes la plupart du temps obliques, souvent subspinuliformes vers le haut, et, a leur parlie inféricure, de striations spirales plus ou moins prononcées.

PLOTIA LEROYI, spec, nor.

Testa imperforata, elongato-oblonga, parum crassula, leviter subdiaphana, uniformiter cornea cum flammulis castaneis continuis aut interruptis inter sulcos, ac eleganter costis transversis modo in ultimo superioribus et ad aperturam evanescentibus) ornata et ad partem inferiorem ullimi striis spiralibus circumcincta; - spira relative elongata, exacte acuminata, ad summum peracuta; - anfractibus 9 convexiusculis, regulariter usque ad ultimum crescentibus, sutura impressa separatis; - ultimo dimidiam altitudinis non attingente, convexo; - apertura verticali, oblonga, superne angulata; columella brevi, crassa, curvata; - peristomate externe acuto; margine externo superne sinuoso, dein valde antrorsum provecto et arcuato;-alt., 18; diam., S; alt. ap., S; lat.. 4 millim.

Les côtes transversales, sur les deux tours antépéunltièmes, sont un tant soit peu subspinuliformes à leur partie supérieure. Le bord externe offre une projection arquée si accentuće que la base columellaire semble comme rétrocédente.

Cette Espèce, dédiée au R. P. Leroy, parait 
abondante dans le fleuve et les cours d'eau de la vallée inférieure du Vouami.

\section{PLOTIA BLOYETI, spec. nov.}

Cette Espèce qui, à première vue, à l'air de ressembler à la précédente, diffère néanmoins essentiellement de celle-ci, par sa taille moitié plus petite (haut., 9 ; diam., 5 ; haut. ouv., 5 ; larg., 5 millim.), par sa forme plus ventrue, moins oblongue, par sa spire moins allongée, tout en ayant une apparence scalariforme assez accusée, par suite d'une plus forte saillie de ses còtes transverses, par les stries spirales de la base de son dernier tour plus prononcées, par son bord externe relativement moins arqué en avant; enfin, par ses tours plus convexes, dont le dernier, plus ventru supérieurement, offre une convexité moins régulière que celui de la Leroyi.

Celte forme, très distincte de la Leroyi, vit dans les cours d'eau des vallées du Vouami et du Kyngani, où elle parait abondante. 


\section{XXIII}

\section{VALVATIDA}

J'ai à faire connaitre une Valvée nouselle, décou verte dans l'Ouébi, en amont de Guélidi. C'est la première Valvée signalée dans les régions équatoriales. On ne connaissait, en effet, jusqu'à présent que 17 Valvées, savoir : 4 en Algérie, 12 en Égypte et 1 au Choa. Voici la liste, par ordre alphabétique, des Valvées africaines:

Valvata egrptaca, Bourguignat, Spec. Moll., $\mathrm{n}^{\circ}$ 195, 1878, et Innes, in : Bull. Soc. malac. Fr., I, p. 350, 1884. - Basse Égypte.

Valvata callista, Bourguignat (loc. sup. cit.), $\mathbf{n}^{0} 196,1878$, et Innes, in : Bull. Soc. malac. Fr. I, p. 350, 1884. - Basse et moyenne Égypte.

Valvata Defevieleusa, Hagenmüller, in : Bull. Soc. malac. Fr., I, p. 213, 1884. - Algérie (env. de Bône).

Valvata Exills, Paladille, in : Nonv. misc. ma-

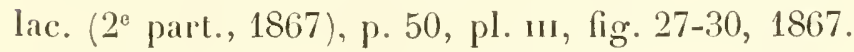
- Agérie (prov. de Constantine).

Valyata Hagexmulere, Bourguignat, in : Bull. Soc. malac. Fr., I, p. 214, 1884.- Algérie (env. de Bòne).

Valvata kuedrialis, Letourncux, in : Innes, in: 
Bull. Soc. malac. Fr., I, p. 348, 1884. - Basse Égypte.

Valvata Letoureuxi, Bourguignat, Spec. Moll., $n^{\circ}$ 194, 1878, et in : Bull. Soc. malac. Fr., I, p. 349, 1884. - Basse Égypte.

Valvata nilotica, Jiclicli, Moll. N.-O. Afrique, p. 233, pl. vil, fig. 29, 1874. - Basse Égypte.

Valvata Petretrixh, Letournelux, in : Bull. Soc. malac. Fr., I, p. 349, 1884. - Basse Égypte.

Valvata Pifaraonum, Bolliguignat, in : Bull. Soc. malac. Fr., I, p. 351, 188' - - Base Égypte.

Valvata Planonbulina, Paladilhe, in : Nouv. mise. malac. (2e part., 1867), p. 49, pl. n, fig. 2326. - Algérie (prov. de Constantine).

Valvata planulata, Bourguignat, Spec. Moll., $n^{0} 177,1878$, et Innes, in : Bull. Soc. malac. Fr., I, p. 351, 1884. - Basse et moyenne Égypte.

Valvata Rothl, Bourguiguat, in: Inues, in: Bull. Soc. malac. Fr., I, p. 347. 1884. - Basse Egrpte.

Valvata Saulcyi, Bourguiguat, Moll. Orient, p. 68, pl. 11, fig. 41-42, 1853, et Innes, in : Bull. Soc. malac. Fr., I, p. 346, 1884. - Moyenne Egypte.

Valvata scionsa, Pollonera, Moll. Scioa, p. 38, 1888. - Choa.

Valvata Swempentm, Bourgulghat, in: Bull. Sor. mal. Fr., 1. p. 352, 18E4. - Moyenne Égyple.

valvata syrica, Bourguignat, Spec. Moll., 
$\mathrm{n}^{0}$ 191, 1878, et Innes, in : Bull. Soc. malac. Fr., I, p. 347, 1884. - Basse et moyenne Égypte.

VALVATA REVOILI (pl. vir, fig. $3-6$ )

Testa profunde umbilicata (perforatio umbilicalis sat aperta), leviter depressa, nihilominus sat globulosa, superne rotundato-perobtusa, subpellucida, cornea, fere laevigata ; - spira brevissima, tholiformi, ad summum rotundata (apex amplus); anfractibus 3 convexis, rapide crescentibus (anfractus embryonalis relative maximus), sutura impressula separatis; - ultimo rotundato, ad aperturam descendente; - apertura leviter obliqua, subsphacrica, superne vix subangulata; - peristomate subcontinuo, undique incrassatulo. - Alt., 3; diam., 4 mil.

Cette Espèce, remarquable par sa forme obtuse, arrondie en dessus en forme de dôme, par son tour embryonnaire relativement énorme, ainsi que par la croissance rapide de ses tours et par la direction descendante de son dernier tour, a été recueillie par le voyageur G. Revoil dans l'Ouébi, en amont de Guélidi, à quelques journées de marche de Moguedouchou. Je ne puis rapprocher cette Valvée d'aucune des formes africaines que je viens de citer et qui toutes me sont connues. 


\section{XXIV}

\section{SPHARID E}

Les Espèces de cette famille ne sont représentées jusqu'à présent que par quelques formes du genre Corbicula. Je ne sache point que l'on ait rencontré des Spharium, des Pisidium ou des Eupera, bien que je ne doute pas de la présence de plusieurs Espèces de ces genres dans ces contrées équatoriales, encore si superficiellement explorées.

\section{CORBICULA}

Les formes de ce genre, toutes recueillies dans le fleuve Kyngani, sont remarquables en ce sens qu'elles ressemblent en tous points aux formes que l'on rencontre dans les canaux et les cours d'eau de la basse Égypte. Il n'y a pas de différences sensibles entre les échantillons de ces provenances si distantes. Ainsi, les Espèces kynganiennes sont les:

Corbicula æegyptiaca, Bourguignat, 1885;

- Degonsei, Bourguignat, 1885;

- subtruncatula, Bourguignat, 1885 ;

que je crois inutile de décrire ici, puisque les descriptions et les figures en sont données dans mon Histoire malacologique de l'Égypte, en cours d'im pression. 


\section{$\mathrm{XXV}$}

\section{UNIONIDA}

Les Unionidées de ces régions équatoriales appartiennent aux genres Pharaonia et Unio.

\section{PHARAONIA}

J'ai reçu, de la vallée du Vouami, une valve de Pharaonie, malheureusement en trop mauvais état pour qu'elle puisse être décrite. Grâce à cette valve, il est acquis actuellement que ce genre existe dans presque toutes les régions du grand Centre zoologique afrieain, puisqu'il a été constaté depuis le Sénégal, à l'Onest, jusqu'en Égypte, en passant par l'Assinie, le Gabon, le Congo et le Zanguébar.

Ce grenre, reconnu par moi en 1878, puis établi par moi en $1880^{\prime}$ et 1888", adopté par plusieurs auteurs, notamment par les $\mathrm{D}^{\text {rs }}$ Jousseaume ${ }^{3}$ et de Rochebrune 4, se compose d'Espèces à test délicat, fort peu inéquilatéral, remarquables par leur apparence extérieure anodontiforme, et caractérisées

1. Matér. Moll. Acéph., I, p. 3, 1880.

2. Iconogr. 'Tang. Explic., pl. xxı.

3. Coq. Haut Sénégal, p. 16 (extr. Bull. Soc. zool. Fr., XI, $1886)$.

4. Lamellibranches Nouv. Congo, in : Bull. Soc. malac. Fr., III, 1886. 
intérieurement par une charnière munie de deu.x lamelles latérales presque aussi longues l'une que l'autre, et ne formant pour ainsi dire qu'une seule par suite de leur jonction, à peine interrompue, à l'endroit des sommets. Chez les Unios, la lamelle antérieure, improprement dénommée dent cardinale ${ }^{1}$, est grosse, courte, épaisse et dentée; la lamelle postérieure seule est allongée.

On peut juger, du reste, des caractères des Pharaonies en examinant les descriptions et les figures des:

Unio Juliani du Sénégal (Rang, Acéph. d'eau donce du Sénégal, p. 10, 1S34).

Unio Essoensis, d'Assinie (Chaper, Esp. nour. coq. vir. Afr. australe et d'Assinie, p. 3, pl. xı, fig. 7-9, 1S85̌).

Pharaonia Bellamyi, du haut Sénégal (Jousseaume, Coq. haut Sénégal, p. 16, pl.xu, fig. 7, 1856).

Pharaonia Bourguignati, du Congo (de Rochebrune, Lamellibr. nouv. Congo, p. 13, 1886).

Etc.....

\section{UNIO}

Les Espèces de ce genre proviennent des bassins du Vouami et du Kyngani; elles ont été mentionnées et décrites par M. Xavier Charmes, dans un excellent travail ${ }^{2}$, publić dans les Bulletins de

1. La vraie dent cardinale nexiste pas chez les Unionidécs.

2. Unionida des envirous de Bagamoyo. 
la Société malacologique de France (tome II, p. 165 et suiv.), en 1885. Ces Espèces sont :

Unio Mossnibicexsis, Peters, mss, in : Martens Pet. Moss. Moll. in : Malak. B1., 1859, p. 218, pl. 11, fig. 3-5, et Charmes, in : Bull. Soc. Malac. Fr., II, p. $166,1885$.

Cet Unio, répandu dans les cours d'eau du bassin du Zambèze, a été retrouvé bien identique et en abondance dans le fleuve Kyngani, ainsi que dans le Vouami.

Unio ratidotus, Charmes, in : Bull. Soc. malac. Fr., II, p. 166, 1885.

Cet Unio, du fleuve Kyngani, se distiugue du Mossambicensis par sa taille un peu moins forte, par sa forme moius haute, par sa région antérieure plus exiguë, moins développée en hauteur et plus fortement décurrente inférieurement; parsa partie rostrale plus étroitement obtuse et regardant plus en bas; par son bord inférieur moins arqué; enfin, notamment par son mode de convexité qui est tout différent.

Unio Dumesnilianus, Charmes (loc. sup. cit.), p. $168,1885$.

Cette Espèce, du Kyngani et de son affluent le Guéringuéré, est remarquable par les rugosités qui couvrent ses valves et par les forts tubercules des sommets; elle se distingue encore des deux précédentes par sa taille moindre, par sa convexité plus forte, par sa crête dorsale plus accentuée, offrant un angle plus saillant, par son contour pos- 
térieur, descendant rectilignement sur une partie rostrale plus inférieure, et regardant bien en bas.

Unio Billottunus, Charmes (loc. sup. cit.) p. 170, 1885 .

Cette Espèce, caractérisée par sa forme ovoïde régulièrement renflée dans toutes ses parties, par ses valves minces, brillantes, très finement striolées, comme polies, par l'amplitude de sa région antéricure, etc., a été découverte dans un ancien bras du Kyngani.

Unio eupruyus, Charmes (loc. sup. cit.), p. 171, 1885.

Ce bel Unio, de forme ovoïde, si richement orné de rides et de tubercules, remarquable par la régularité de sa convexité el par ses sommets peu proéminents, comme écrasés, a été également découvert dans le cours du Kyngani.

Unio Lejoulxianus, Charmes (loc. sup. cit.), p. 173,1885 .

Cette Espèce, la plus allongée des Únios de cette région, vit dans les cours d'ean des bassins du Kyngani et du Vouami. 


\section{XXVI}

\section{IRIDINID小}

Les Iridinidées, dont les formes sont si multipliées et si développées dans le lac Tanganika, ne sont représentées, à ma connaissance, dans les contrées équatoriales, intermédiaires entre ce lac et l'océan Indien, que par quatre Espèces du genre Spathella.

Ce genre, établi par moi en 1885', aux dépens dugenre Spatha, dans lequel il avait été confondu, se compose d'Lispèces oblongues-allongées, i valves légères, assez minces, à charnière filiforme munie d'une éminence cardinale sénestre très réduite.

Parmi les Spathelles conmues du continent, je mentionnerai les :

1. Espèces nouvelles et genres nonveaux découverts par les IRR. PP. missionnaires dans les grauds laes africains Oukéréwé et Tanganika, p. 14. - Dans ce travail, j’ai divisé l'ancien geure Spatha en trois conpes génériques: 10 Spatia, pour les grosses Especes subarrondies ou obtuso-oblongues, a valves épaisses, pesantes, à charnière robuste, édentulée, offrant seulement sur la valve gauche, au niveau de la dent cardinale, une éminence tuberculeuse très émoussée el très allongée (type Spatha rubens du Nil); $2^{\circ}$ Spatuelea pour les Espèces allongées, de taille médiocre (type Spathella Pelersi de Mozambique et du Zangurbar); $3^{\circ}$ Aspatuaria pour les Espèces de petite taille, à région postérieure nettement tronquée, à eharnière exiguë avec un sentiment d'éminence tuberculeuse sur la valve gauche, et reinarquables par leurs valves couvertes de rides et de rugosités (type Aspatharia Vignouana du haut Gabon). 
Spatuella sivuata, Bourguignat (Spatha sinuata, Martens, Afrik. binnenconch, in : Conch. Mittheil., II (fasc. 5-6), p. 190, pl. xxxiv, fig. 5-6, 1885. - Affluents du Congo.

Spatiella Waildergi, Bourguignat (Iridina Wahlbergi, Krauss, sudafr. moll., p. 19, pl.ıI, fig. 1, 1848; - Spatha Wahlbergi, Martens, Mossamb. Moll. in: Malak. Bl., 1859, p. 217. - Fleuve des Singes (Affenfluss); affluent du Limpopo; Tette sur le Zambèze.

Spathella Protchei, Rochebrune, Lamellibr. Congo, in : Bull. Soc. malac. Fr., III, 1886, p. 8. - Mokaka, sur le Congo.

Spatiella Adansoni, Jousseaume, Coq. haut Sénégal, p. 28, pl. xıv, fig.4-4ª 1886.-Le Diamonko, affluent du Sénégal.

Spathella myassana, Bourguignat (Spatha Nyassaensis, Lea, in: Proc. acad. nat. sc. Philad., 1864. p. 109, et in : Journ. acad. nat. sc. Philad., p. 36, pl. xıI, fig. 33, 1866. - Lac Nyassa (Mozambique).

Spathella Pfeifferian, Bourguignat, Esp. nouv. grands lacs afr., p. 14, 1885 (Margaritana Pfeifferiana, Bernardi, in : Journ. conch., VIII, 1859, p. 331, pl. xir, fig. 1-2). - Cours d'eau du Gabon.

Ete., Etc.....

Les quatre Spathelles des régions ousaghariennes que j'ai à faire connaître sont : 
SPATHELLA PETERSI

Spathella Petersi, Bourguignat, Mossamb. Moll. in : Malak. Bl., 1859, p. 218, pl. Iu f. 1-2.

Cette Espèce, de forme oblongue-allongée dans le sens horizontal, primitivement découverte dans le Zambèze, aux environs de Tette, a été retrouvée parfaitement typique dans les cours d'eau de la haute vallée du Vouami, aux alentours de Kondoa, notamment dans le Kondokoua et le Makata.

\section{SPATHELLA BOLRGUIGNATI}

Spathella Bourguignati, Ancey, in litt., et in : Bull. Soc. malac. Fr., IV, 1887, p. 268 (olim Spatha Bourguignati, Ancey, in : Bourguignat, Esp. nouv. grands lacs afric., p. 12, 1885, et pl. vill, fig. 1.2).

Cette Spathelle, recueillie d'abord sur la côte méridionale de l'Oukéréwé, a été constatée dans les cours d'eau de la plaine marécageuse d'Hahi, entre l'Ougogo et Tabora. Elle se distingue de la Spathella Petersi, par son bord inférieur non rectiligne, mais légèrement convexe; par sa région antérieure plus ample et sensiblement plus accentuée à sa partie supérieure; par ses sommets plus obtus; par son épiderme d'une coloration différente, ainsi que par sa nacre interne d'une autre muance; par ses valves non bâillantes (chez la $P e-$ tersi, les valves oflrent deux étroits bàillements en avant et en arrière). 


\section{SPATHELLA BLOYETI}

Spathella Bloyeti, Bourguignat, pl. vur, fig. 3.

Long. max., 83 ; alt. max. . 42 ; alt. vert., 40 ; crass. max.. 22 ; basis apico-rostralis, 65 ; intervallum e natibus ad angulum et ex angulo ad rostrum aque, 35 ; e rostro ad verticalem, 51 , ac e basi verticalis ae angulum, 47; regio antica, 28; postica, 56 millim.

Concha inæquilaterali, elongato-suboblonga in directionem vix declivem, undique obtusa, superne c parte antica ad rostrum exacte convexoarcuata, inferne vix decliviter subrectiuscula, parum nitente, rustice striata, uniformiter obscure straminco-lutea, intus albo-margaritacea et ad nates leviter subaurantiaca.

Valvis mediocriter crassis, vix antice posticeque bihiantibus (hiantixe angustissima), parum convexis (convexitas in ventre valvarum sat plana, nihilominus convexitas maxima ad 15 verticalis sita, et e natibus et ex angulo xqualiter 21 , e rostro 44, ex antico margine 41 , et e basi verticalis 28 millim. remota).

Supra arcuata usque ad angulum, dein convexodescendente; antice sat ampla, rotundata; infra subrecliuscula ac nihilominus in medio obscure subsinuata; postice (regio postica duplicem anterioris aquans ac inferne in altitudinem usque ad 23 verticalis leviter crescens) in rostrum obtusum infernumque attenuata.

Umbonibus mediocriter anticis perobusis, vix 
convexis, potius leviter obtritis, super erosis; area mediocri, ad angulum obtusum vix compressa; ligamento posteriori parum prominente, atro, fere semitecto.

Cette Espèce, dédiée au commandant Bloyet, vit dans le Makata, affluent supérieur du Vouami (Ousaghara).

Parmi les Spathelles, je ne vois que la Wahlbergi qui puisse lui être comparée; mais cette dernière est le double plus grande; son épiderme est olivâtre; son bord supérieur est moins arqué; ses sommets sont un peu plus proéminents; ses valves offrent vers la partie inféro-médiane une dépression sinueuse accentuće, sinuosité à peine sensible chez la Bloyeti; enfin, sa région rostrale est moins inférieure.

\section{SPATHELLA SPATHULIFORMIS}

Spathella spathuliformis, Bourguignat, pl. vir, fig. 4 .

Long. max., 97 ; alt. max., 52 ; alt. vert., 49; crass. max., 31 ; — basis apico-rostralis, 73; intervallum e natibus ad angulum, 3S; ex angulo ad rostrum, 40; e rostro ad verticalem, 60 ; et e basi verticalis ac angulım, 52 ; - regio antica, 35 ; postica, 6́ millim.

Concha inaquilaterali, oblongo-spathuliformi in directionem declivem, postice notabiliter ampla; undique rustica, striata, uniformiter luteo-straminea ct intus albo-margaritacea. 
Valvis crassis, ponderosis, vix hiantibus (hiantia postica perangusta), inferne subsinuatis, relative sat convexis (convexitas maxima sat superior, ad 15 verticalis sita, et e natibus 22 , e rostro et ex antico margine aque 50, ex angulo 21, et e basi verticalis 35 , remota).

Supra primo subrecta, dein convexo-descendente; antice rotundata; infra decurrente in medio leviter subsinuosa; postice ampla, nihilominus duplicem regionis anterioris non aequante, usque ad 29 verticalis in altitudinem leviter crescente ac in rostrum obtusum inferumque attenuata.

Umbonibus perobtusis, parum prominentibus; regione sulci dorsali ventroso, superne fere abrupte super aream (area nulla) descendente; ligamento valido, atro, prominente.

Cette Spathelle se distingue de la précédente par sa taille pliss grande; par sa région postérieure plus développée, présentant une apparence spathuliforme; par ses valves plus épaisses, plus pesantes, bâllantes seulement en arrière; par la sinuosité accusée de son bord inférieur; par son mode de convexité différent. Chez la spathuliformis, en effet, la convexité parait plus sensible vers le haut, notamment sur la région postéro-dorsale, et offre une descente presque abrupte sur le bord supérieur; chez la Bloyeti, cette descente est régulière, par suite d'une répartition plus normale du mode de convexité.

Celle Espèce a été recueillie dans le cours d'eau Magogo, à Ounyangouira (Ougogo). 
XXVII

RÉSUMÉ MÉTHODIQUE

DES ESPÉCES SIGNALĖES

DE MOGUEDOUCHOU A BAGAMOYO

ET

DE BAGAMOYO $\triangle$ U TANGANIKA

\section{GASTEROPODA INOPERGULATA}

\section{$1^{\circ}$ PUL MONACEA}

UROCYCLIDE

Urocyclus Kirki, Gray.

HELIXARIONIDE

Tapsia Leroyi, Grandidier, p. 12.

Moaria Chaperiana, Bourguignat, p. 14.

Trochonanina Mozambicensis, Mousson, p. 17.

- Ibuensis, Martens, p. 18.

- Leroyi, Bourguignat, p. 18, pl. ", fig. $13-14$.

- Jenynsi, Martens, p. 19.

1. Je n'ai pas mentionné l' Crocyclus Kirki, qui a été recueilli par le capitaine Bloyet, aux environs de Kondoa (Ousaghara). Je renvoie pour la connaissance de cette Espèce au savant Mémoire publié par notre ami le Dr Poirier (Bull. Soc. malac. Fr., IV, 1887, p. 195.232, el pl. v i x) sur le genue Urocyelus. 
Trochonanina percarinata, Martens, p. 19.

- Anceyi, Bourguignat, p. 20.

- Bloyeti, Bourguignat, p. 21, pl. 11, fig. 10-12.

Hamya Revoili, Bourguignat, p. 23.

Ledoulxia albopicta, Bourguiguat, p. 24.

- pyramidxa, Bourguignat, p. 25.

- formosa, Bourguignat, p. 25.

- megastoma, Bourguignat, p. 26.

- insignis, Bourguignat, p. 26.

- unizonata, Bourguignat, p. 27.

Bloyetia Revoili, Bourguignat, p. 30.

- magnifica, Bourguignat, p. 31.

- carulans, Bourguignat, p. 31.

- compressa, Bourguignat, p. 32.

- Georgi, Bourguignat, p. 32.

- Rochebruniana, Bourguignat, p. 32.

- Mabilliana, Bourguignat, p. 34.

- Leroyi, Bourguiguat, p. 34.

\section{ARTEMONID A:}

Gibbonsia gigas, Bourguignat, p. 38.

Tayloria ventrosa, Bourguignat, p. 39.

- Jouberti, Bourguignat, p. 39 et 47, pl. ॥, fig. 6-9.

Colpanostoma Leroyi, Bonrguignat, p. 43 et 48 , pl. I, fig. 1-3.

BULININIDE

Bulimus Lourdeli, Bourguignat, p. 51, pl. "1, fig. 1-3. 
Bulimus Bridouxi, Bourguignat, p. 53, pl. 1 , fig. 4-5.

\section{ACHATINID.E}

Achatina Marioni, Ancey, p. 74.

- panthera, Deshayes, p. 75.

- zanzibarica, Bourguignat, p. 75.

- Lhotellerii, Bourguignat, p. 75.

- Craveni, Smith, p. 76.

- Letourneuxi, Bourguignat, p. 79.

- Milne-Edwardsi, Revoil, p. 79.

- reticulata, Pfeiffer, p. 79.

- Bloyeti, Bourguignat, p. 79 et 82.

- arctespirata, Bourguignat, p. 80 el 83, pl. v, fig. 7.

- Randabeli, Bourguignat, p. 80 et 84 , pl. v, fig. 6 .

Serpar Pintoi, Bourguignat, p. 86, pl. w, fig. 4.

Burtoa Reymondi, Bourguignat, p. 92, pl. w, fig. 1.

- Bridonxiana, Bourguignat, p. 92, pl. w, fig. 3 .

- sebasmia, Bourguignat, p. 93, pl. ın, fig. 1.

- Pethericki, Bourguignat, p. 95.

- Lavigeriana, Bourguignat, p. 96, pl. ir, fig. 2 .

Burtopsis Giraudi, Bourguignat, p. 98, pl. v, fig. 1.

- Jouberti, Bourguignat, p. 99, pl. 11, fig. 1.

Limicolaria Charbonnieri, Bourgugnat, p. 102 et 104, pl. ve fig. 7-8.

- Spekiana, Grandidier, p. 102. 
Limicolaria megalxa, Bourguignat, p. 102 et 105, pl. vi, fig. 4.

- Coulboisi, Bourguignat, p. 103 et 106, pl. vi, fig. 1.

- Dromauxi, Bourguignat, p. 103 et 107, pl. vi, fig. 3.

- sepulcralis, Bourguignat, p. 103 et 108, pl. vi, fig. 2.

- rectistrigata, Grandidier, p. 103.

- Burtoniana, Grandidier, p. 103.

- Bridouxi, Grandidier, p. 103.

- Martensiana, Crosse, p. 104.

- Giraudi, Bourguignat, p. 104, pl. v, lig. 5 .

- lamellosa, Bourguignat, p. 104 et 109, pl. vi, fig. 6 .

Rachis Cameroni, Bourguignat, p. 58.

- Spekei, Bourguignat, p. 59.

- Hildebrandti, Martens, p. 59.

- rhodotrenia, Martens, p. 60.

- picturatus, Bourguignat, p. 60 .

- Bloyeti, Bourguignat, p. 60.

- Jouberti, Bourguignat, p. 61, pl. III, fig. 10-12.

- pachistoma, Bourguignat, p. 62, pl. II, fig. 8-9.

- elongatulus, Bourguignat, p. 63, pl. IIl, fig. $4-5$.

Pachnodus Leroyi, Bourguignat, p. 65, pl. III, fig. $4-5$.

- sesamorum, Ancey, p. 66, pl. uI, fig. 2-3. 
Rachisellus punctatus, Bourguignat, p. 69.

- Burtoi, Bourguignat, p. 69.

- variolosus, Bourguignat, p. 70.

- Ledoulxi, Bourguignal, p. 70 , pl. v, fig. 10-11.

\section{STENOGYRIDE}

Stenogyra Leroyi, Bourguignat, p. 110, pl. vi, fig. 2. - Grandidieriana, Bourguignat, p. 111, fig. vi, fig. 9-10.

Subulina Mabilliana, Bourguignat, p. 114.

- lenta, Smith, p. 114.

- intermedia, Gibbons, p. 114.

- cylindracea, Bourguignat, p. 115, pl. v, fig. 2-3.

- Jouberti, Bourguignat, p. 115, pI. v, fig.4.

\section{CLAUSILIDA}

Clausilia Giraudi, Bourguignat, p. 117, pl. v, fig. 8-9.

\section{STREPTOSTELIDE 1}

Anceya Girandi, Bourguignat, p. 118, pl. vn, fig. 12-13.

- admirabilis, Bourguignat, p. 119, pl. vil, fig. 10-11.

\section{ENNEIDE}

Enneastrum levigatum, Bourguignat, p. 127.

1. C'est avec doute que je classe le genre Anceya dans cette famille. 
STREPTAXID.E

Gonaxis Gibbonsi, Taylor, p. 133.

- Mozambicensis, Bourguignat, p. 133.

- Craveni, Bourguignat, p. 134.

- Bloyeti, Bourguignat, p. 134, pl. vit, fig. 1-3.

Marconia lata, Bourguignat, p. 136.

- conoidea, Bourguignat, p. 137.

- gibbosa, Bourguignat, p. 137, pl. vir, fig. 6-7.

- recta, Bourguignat, p. 138, pl. vin, fig. 4-5.

Edentulina ovoidea, Bourguignat, p. 140.

- obesa, Bourguignat, p. 141.

- bulimiformis, Bourguignat, p. 141.

- Grandidieri, Bourguignat, p. 142, pl. vil, fig. 8-9.

\section{$2^{\circ}$ PULMOBRANCHIATA}

\section{LIMNAIDE}

Limnaca africana, Ruppell, p. 157.

- Debaizei, Bourguignat, p. 157.

- truncatula, Goupil, p. 157.

- Cameroni, Bourguignat, p. 157.

- Kynganica, Bourguignat, p. 158.

- Zanzibarica, Bourguignat, p. 158.

Physopsis Stanleyana, Bourguignat, p. 160.

- preclara, Bourgnignat, p. 160.

- ovoidea, Bourguignat, p. 160.

- Soleilleti, Bourguignat, p. 160. 
Physopsis Leroyi, Grandidier, p. 160.

- Bloyeti, Bourguiguat, p. 160.

\section{GASTEROPODA OPERGULATA}

\section{$1^{\circ}$ PULMONACEA}

\section{GYCLOSTONIDE}

Georgia naticopsis, Bourguignat, p. 143.

- Guillaini, Bourguignat, p. 144.

- Perrieri, Bourguignat, p. 144.

- Poirieri, Bourguignat, p. 144.

- Revoili, Bourguignat, p. 144.

Rochebrunia Guillainopsis, Bourguignat, p. 146.

- Delmaresi, Ancey, p. 146.

- insularis, Bourguignat, p. 146.

- Zanguebarica, Bourguignat, p. 147.

- obtusa, Bourguignat, p. 147.

Maizania olivacea, Bourguignat, p. 149, pl. vu, fig. 14-18.

Cyclostoma anceps, Martens, p. 150.

- Cambieri, Bourguignat, p. 150.

- lineatum, Pfeiffer, p. 151.

- Letourneuxi, Bourguignat, p. 152.

- Leroyi, Bourguignat, p. 152, pl. vil, lig. 19-20. 


\section{$2^{\circ}$ BRANCHIATA}

\section{PALUDINIDA}

Vivipara capillata, Frauenfeld, p. 162.

- Robertsoni, Frauenfeld, p. 162.

- Jeffreyssi, Frauenfeld, p. 163.

Cleopatra Letourneuxi, Bourguignat, p. 163.

- Cameroni, Bourguignat, p. 163.

- Kynganica, Bourguignat, p. 164.

- africana, Bourguignat, p. 164.

- exarata, Bourguiguat, p. 164.

- Zanguebarica, Bourguignat, p. 164.

- Pauli, Bourguignat, p. 165.

- Ajanensis, Bourguignat, p. 165.

- Guillemeti, Bourguignat, p. 165.

ANPULLARIDE

Ampullaria Dumesniliana, Billotte, p. 169.

- gradata, Smith, p. 169.

- Letourneuxi, Bourguignat, p. 169.

- Revoili, Billotte, p. 169.

- Ruchetiana, Bourguiguat, p. 169.

- $\quad$ speciosa, Philippi, p. 169.

Meladomus purpureus, Bourguignat, p. 170.

- Bloyeti, Bourguignat, p. 171

- pyramidalis, Letourneux, p. 171.

- nitidissimus, Bourguignat, p. 171.

- procerus, Bourguignat, p. 172.

- elatior, Bourguignat, p. 173.

- ovum, Bourguignat, p. 173. 
Meladomus Jouberti, Bourguignat, p. 174.

sinistrorsus, Bourguignat, p. 174.

- affinis, Bourguignat, p. 175.

- solidus, Bourguignat, p. 175.

- Deguerryanus, Bourguignat, p. 175.

- ciliatus, Bourguignat, p. 177.

- Alexandri, Bourguignat, p. 177.

- Boltenianus, Bourguignat, p. 178.

- Duveyrierianus, Bourguignat, p. 179.

Leroya Bourguignati, Grandidier, p. 180.

- Charmetanti, Grandidier, p. 180, pl. vin, fig. 21-22.

\section{MELANIDE}

Melania tuberculata, Bourguignat, p. 182.

- Inhambanica, Martens, p. 183.

Tiara Vouamica, Bourguignat, p. 183.

Plotia Leroyi, Bourguignat, p. 185.

- Bloyeti, Bourguignat, p. 186.

\section{VALVATIDE}

Valvata Revoili, Bourguignat, p. 189, pl. vill. fig. 5-6.

\section{LAMELLIBRANCHIATA}

\section{SPHERIDE}

Corbicula agyptiaca, Bourguignat, p. 190.
- Degousei, Bourguignat, p. 190.
- subtruncatula, Bourguighat, p. 190. 
UNIONIDE

Pharaonia (spec.), p. 191.

Unio Mossambicensis, Peters, p. 193.

- ratidotus, Charmes, p. 193.

- Dumesnilianus, Charmes, p. 193.

- Billotianus, Charmes, p. 194.

- euphymus, Charmes, p. 194.

- Ledoulxianus, Charmes, p. 194.

\section{IRIDINID $E$}

Spathella Petersi, Bourguignat, p. 197.

- Bourguignati, Ancey, p. 197, pl. vill, fig. 1-2.

- Bloyeti, Bourguignat, p. 198, pl. vill, fig. 3 .

- spathuliformis, Bourguignat, p. 199, pl. vin, fig. 4. 


\section{TABLE ALPIIABÉTIQUE}

DES

\section{NOMS GÉNÉRIQUES, SPÉCIFIQUES}

ET

SYNONYMIQLES

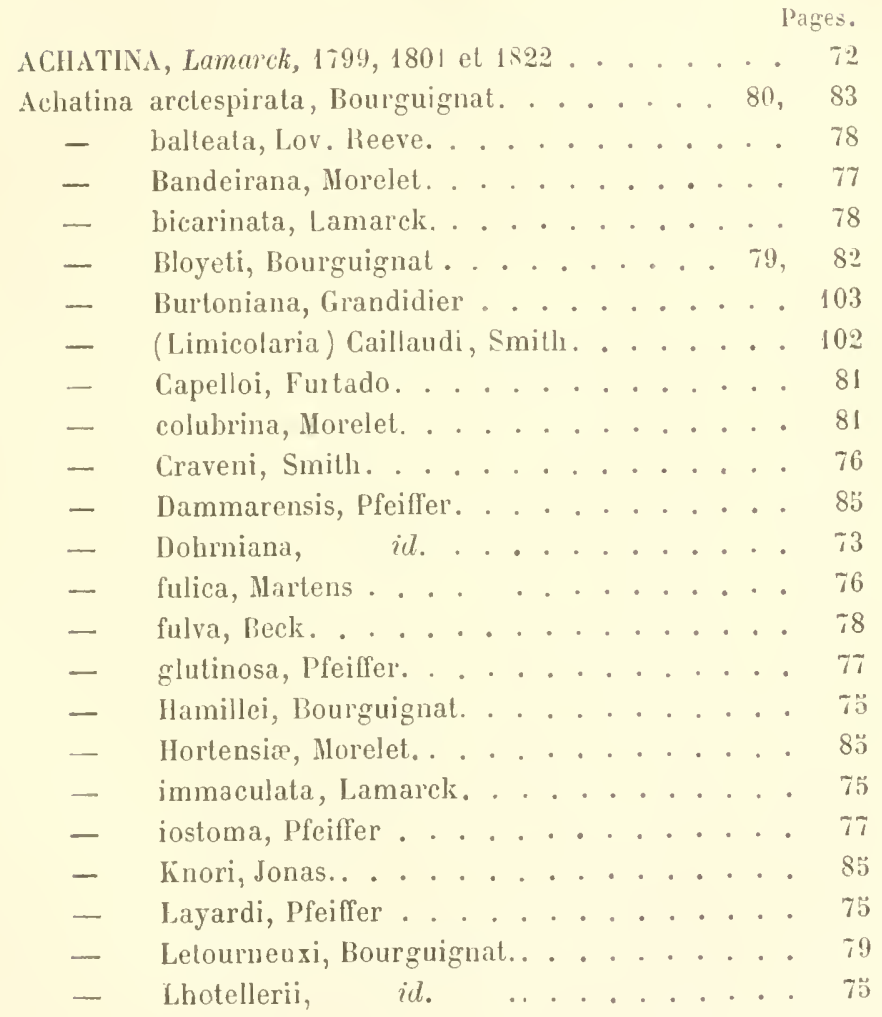


Achatina Marioni, Ancey.......... 74

- (Limicolaria) Martensiana, Smith. ..... 10'

- monetaria, Morelet.. . . . . . . . . 75

— Milne-Edwardsi, Révoil. ......... 79

- Paivana, Morelet. .......... 77

- panthera, Deshayes. . . . . . . . 75

- perdrix, Lantarck. . . . . . . . . 7t

- perfecta, Morelet.. . . . . . . . . 78

- Petersi, Martens. . . . . . . . . . 78

- Pfeifferi, Dunker........... 81

- Planti, Pfeiffer.......... 79

- polychroa, Morclet.......... 81

- porphyrostoma, Shuttleworth........ 77

- prunum, Lov. Reeve, . . . . . . . . 85

- purpurea, Lamarck. . . . . . . . . . 77

- Randabeli, Bourguignat. . . . . . 80, 84

- (Limicolaria) rectistrigata, Smitl. ...... 103

- (id.) id. (pars) id. . . 103

- (id.) id. (alt. pars) id. . 103

- reticulata, Pfeiffer ......... 79

- rhodostoma, Philippi .......... 77

- Rodatzi, Dunker. . . . . . . . 76

- Schweinfurthi, Hartens. . . . . . . . . 75

- semidecussata, Menke.. . . . . . . 81

— semisculpta, Pfeiffer......... 80

- sinistrorsa, $i d . \ldots . . . . . . .78$

— specularis, Norelet ......... 80

- suturalis, Philippi........... 78

- Thomsoni, Smith. .......... 73

- ustulata, Lamarck. . . . . . . . . . 80

- varicosa, Pfeiffer. ....... 76,86

- variegata, Roissy. ........... 74

- Vignoniana, Morelet. .......... 73

- Welwitschi, id. ......... 74

. zanzibarica, Bourguignat. . . . . . . . 73

- zebra, Lamarck. . . . . . . . . 76 
Pages.

Achatina zebriola, Morelet . . . . . . . . . . 81

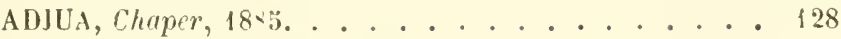

AFRICARION, Godwir-Austen, 1s83......... 11

ALCID]A, Bourguignat, 1889. . . . . . . . . . 46

Aleidia alveus, Bourgnigrat. . . . . . . . . . . 47

- aperta, id. ........... . 47

- cyprele, ril. ............. 47

- l'aivana, il. ............ 47

Amnonoceras ammonitiformis, Doring . . . . . . 43

AMPULLARIA, Lamarck, 1879 et 1801 . . . . . . . 166

Ampullaria adusta, Lov. Reeve . . . . . . . . . 166

- acyptiaca, Ehrenberg. . . . . . . . . 179

- balenoidar, Gould . . . . . . . . 166

- Bernardiana, Norelet . . . . . . . 170

.- Bolteniana, Philippi. . . . . . . . . 179

- Bourguignati, Billotte. . . . . . . 166

- Bridouxi, Bourguignat. . . . . . . . 166

- carinata, Lamarck. . . . . . . . . . 179

- Cluarmesiana, Billotte. . . . . . . 167

- Dumesniliana, $i d . \quad . . . . . . \quad 167,169$

- elliptica, Martens . . . . . . . . 173

- erysthrostoma, Lov. Reeve. . . . . . . 167

- exigua, Plilippi. ........... 167

- gradala, Smill.. . . . . . . . 167, 169

- Grasseti, Morelet ............ . 172

- Guinaica, Lamarek . . . . . . . . . . 175

- lolostoma, Murelet . . . . . . . 176

- intorta, Lamal’ck. . . . . . . . . 174

- Kordofana, rarreyss. . . . . . . . . 167

- Largillicti, Philippi ........... 167

- Letourneuxi, Bonrguignat. . . . 167, 169

— libyea, Norelet............ 176

- lucida, Parreyss. . . . . . . . . . 167

- nilotica, Sowerby. . . . . . . . . 176

- occidentalis, Mousson . . . . . . . . 167

- ovata, Olivier. . . . . . . . . 168 
Pages.

Anpullaria ovata, Morelet ....... . . . $\mathbf{1 6 8}$

- ovum, Philippi .......... . 173

- id. Peters. . . . . . . . . 173

- purpurea, Jonas. .......... 170

— Raymondi, Bourguignat. ........ 168

- Revoili, Billotte. ........ 168, 169

- Rucheti, id.......... 168, 169

- sinistrorsa, Philippi......... 174

- speciosa, id. ....... 168, 169

- subcarinata, Sowerby. . . . . . . 177

- vilrea, Lov. Reeve. . . . . . . . . 168

- Welwitschi, Bourguignat. . . . . . 168

- Wernei, Plilippi .......... 168

ANCEYA, Bourguignat, 1885. . . . . . . . 118

Anceya admirabilis, Bourguignat . . . . . . 119

- Giraudi, id. . . . . . . 118

AliTENON, Beck, 1838............ 44

Artemon candidus, Beck. . . . . . . . . . 44

- costulosus, Bourguignat. . . . . . . 45

- intermedius, id. . . . . . . . 4t 4 t.

- uberiformis, id. ......... 4 . .

- Wagneri, $i d . \quad . . . . . . .45$

BLOYET1A, Bourguignut, 1889......... . . 28

Bloyelia carulans, Bourguignat. . . . . . . . . 31

- compressa, id. ......... 32

- Georgi, id. ........ 32

- Leroyi, ill. . . . . . . . . 34

- Mabilliana, id. ......... 34

- magnifica, il. ......... 31

- Revoili, id. ........ . 30

- Rochebruniana, $i d$. . . . . . . . . 33

Burcinum truncatulum, Nüller. . . . . . . . . 157

- variegatum exoticum, Columna ..... . 74

Buliminus Brauusi, Martens. . . . . . . . . 59

- id. var. Ilildebrandti, Martens. . . . . 59

— palleus, l'feiller........... 58 


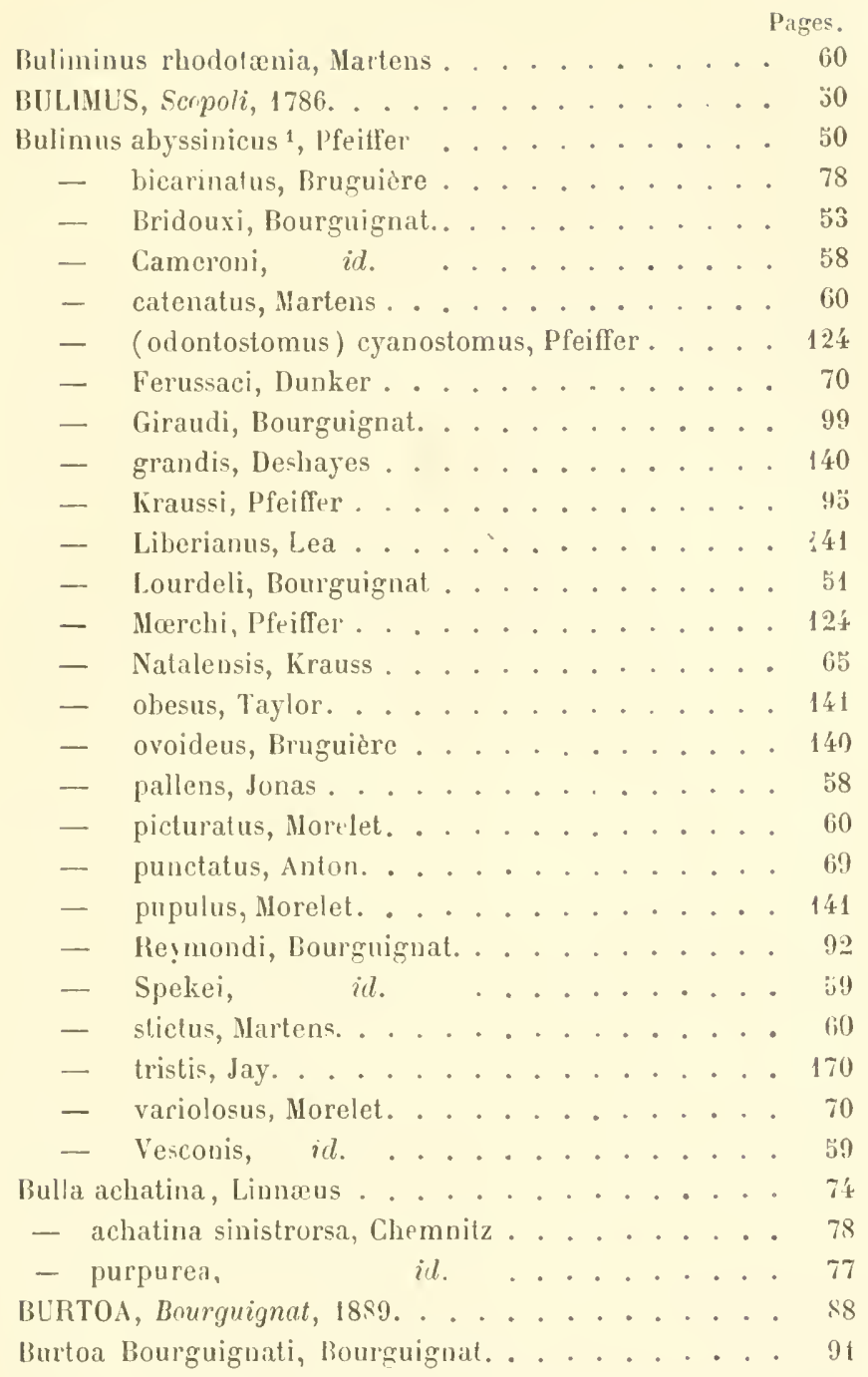

1. Pour la liste des Bulimes du groupe de l'Abyssinicus, voir pages 50 et 51 . 
Pages.

Burtoa Bridouxiana, Bourguignat. . . . . . . 92

- hraussi, $\quad i d . \quad \ldots . . . . . .95$

- Lavigeriana, $\quad i d . \quad \ldots . . . . . .996$

一 nilotica, $\quad i d . \quad \ldots . . . . . .89$

- Pethericki, id. ........ 9ä

- Reymondi, $\quad i d . \quad \ldots . . . . . .92$

- sebasmia, $\quad$ id. . . . . . . . 93

BURTOPSIS, Bourguignat, $1889 \ldots . . . .99$

Burtopsis Giraudi, Bourguignat . . . . . . . . . 9 . . .

- Jouberti, $i d . \quad \ldots . . . . . . .99$.

CLAUSILIA, Draparnaud, 1§03̈. . . . . . 117

Clausilia Giraudi, Bourguignat. . . . . . . . . 117

CLEOPATRA, Trochel, 1857......... 163

Cleopatra africana, Bourguignat. . . . . . . 164

- Ajanensis, $\quad \imath$. . . . . . . 165

- Cameroni, id. ......... 163

- exarala, id. ........ 164

- Guillemeti, $i d . \quad \ldots . . . . .165$

- liynganica, $\quad$ d. . . . . . . . 164

- Letourneuxi, id. ........ 163

- Pauli, $\quad$ id. ......... 163

- Zanquebarica, $i d . \quad \ldots . . . . . .164$

Cochlea Guinaica, Chemnitz . . . . . . . 175

COLPANOSTOMA, Bourguignat, 18\$9. ....... 43

Colpanostoma Leroyi, Bourguignat . . . . . 43, 48

Conulus Adansoniæ, Pfeiffer . . . . . . . . 16

CorbiClla, Mégerle, 1811.......... . . . 190

Corbicula egyptiaca, Bourguignat. . . . . . . 190

- Degonsei, id. . . . . . . . 190

- subtruncala, id. ......... 190

Gyclodontina Guineensis, Beck . . . . . . . 124

CYCLOSTOMA, Draparnaud, 1801 el 1805. . . . . . 150

Cyclostoma anceps, Martens. . . . . . . . . 150

- Cambieri, Bourguignat . . . . . . $1 \ddot{0} 0$

- carinata, Olivier . . . . . . . 178

- Guillaini, Petit .......... 144 
Pages.

Cyclostoma Guillaini, Гfeiffer . . . . . . 146

- insulare, id. ......... 147

- id. Smith ......... 150

- Leroyi, Bourguignat. ........ 152

- Letourneuxi, $i d_{0}$. . . . . . . 152

- lineatum, Pfeiffer ....... 151

- zanguebaricum, id. ....... 147

Cyclostomus insularis, $i d . \quad . . . . . .147$

- Zanguebaricus, id. ....... 147

DISCARTEMON, Pfeiffer, 1850̈........ 4 . .

Discartemon Crossei, Bourguignat ....... 46

- cryptodon, id. ....... 46

- discus, $i d$. ...... 46

DREPANOST0HELLA, Bourguignat, 1889..... 42

Drepanostomella ammonitiformis, Bourguignat. . . . 43

EDENTUliNA, Pfeiffer, 185\%. . . . . . 127, 139

Edeniulina anodon, Bourguignat ...... 141

- arenicola, id. ....... 141

- bulimiformis, il. ...... 141

- Grandidieri, $i l$. ...... 142

- insignis, id. ...... 140

- intermedia, id. ....... 140

- Liberiana, id. ....... 141

- minor, il. ....... 141

- obesa, id. ....... 141

- ovoidea, i....... 140

- tumida, id. ........ 140

ELWA, II. Adams, 1866. . . . . . . . . . 127

E.NEA, II. et A. Adams, 1855 . . . . . . . . 122

Ennea anodon, Pfeiffer. .......... 141

- arenicola, Morelet. . . . . . . . 141

- bicolor, 11. et A. Adams. . . . . . . . 123

- bulimiformis, Grandidier. . . . . . . 141

- cylindrica, pfeiffer. . . . . . . . 123

- insignis, $i d . \quad \ldots . . . . . .140$

- intermedia, Morelet. . . . . . . . 140 
Ennea liprigata, Dohrn. Pates

- lata, Smith............. 136

- Liberiana, Pfeiffer. ........... 141

- minor, Morelet........... 141

- mueronata, Martens ......... $12 \dot{4}$

- Pirriei, Pfeiffer........... 123

- (gonospira) ringens, II. et A. Adams . . . . 194

- tumida, Morelet.......... 140

- vitrea, il. . . . . . . . 136

- zangueharica, id. ......... 1/1

ENTEASTHUM ${ }^{1}$, Pfeiffer, 18\%ั. . . . . . . . . 12.3

Enneastrum levigatum, Bourguignal. . . . . . 127

GEORGIA, Bourguignat, 18.2 ........ 143

Georgia Guillaini, Bonrguinnat......... 144

- naticopsis, id. ......... 143

- Perrieri, ill. ........ 14t

- Poirieri, id. .......... 144

一 Revoili, id. . . . . . . . 14 t'

GIBBONSIA, Bouryuignat, 188? . . . . . . . 38

Giblonsia gigas, Bourguignat. . . . . . . . . $3^{\text {}}$

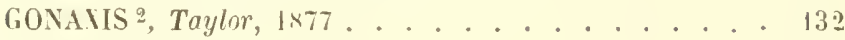

Gonaxis Bloyeti, Bourguignat. . . . . . . . 134

GUILlaINI, Burguignat, 1883. . . . . . . . 23

Guillainia cerulans, Bourguignat . . . . 31

- compressa, $\quad i$. . . . . . 32

- Georgi, il. ...... 32

- Mabilliana, $i \iota$. ..... $3 i$

- magnifica, $\quad i d . \quad \ldots . . . .31$

- Revoili, id. ...... 30

- Rocliebruniana, i...... . 33

Gulella Assiniensis, Chaper. . . . . . . . . 125

- Elimensis, $i$ il. . . . . . . . 123

- Treichi, id. .......... 125

1. Voir, à la page 127, la longue liste des Enneastrum.

2. Voir, a la page 133, la liste des Espèces de ce genre. 
Pages.

HAHYA, Bourguignat, 1883. . . . . . . . . . 23

IIamya Revoili, Bourguignat . . . . . . . . . 23

HAPPL, Bourguignat, $1 \times 89$. . . . . . . . . 39

Happia Cuzcana, Bourguignat ....... 40

- flora, il. ...... 40

- Noyobambensis, $i d . \quad \ldots . . . . .40$

- omalomorpha, id. ...... 40

- orbicula, $\quad$ id. . . . . . . 40

- trochilioides, $\quad$ id. $\quad \ldots . . . .40$

- vitrina, $\quad i d . \quad$. . . . . . 40

Helix achtephila, d’orbigny. . . . . . . . 49

— Adansonir, Morelet. . . . . . . 16

- ammonitiformis, d'(lrbigny ........ 43

- ammonoceras, Pfeiffer .......... . 41

— Baezensis, Hidalgo . . . . . . . . . 40

- Bolteniana, Chemnitz. . . . . . . . 178

- Borbonica, Férussac ......... . 76

- Bounobæna, d'Orbigny .......... 42

- candida. Moricand ............ 44

- chalicophila, d'orbigny ........... 41

- coffreana, Moricand . . . . . . . . . 45

- costulosa, Pfeiffer . . . . . . . . . 45

- (streptaxis) cryptodon, Moricand. . . . . . . 46

- Cuzcana, Philippi. . . . . . . . . 40

- cypsele, Pfeiffer . . . . . . . . . . 47

- elevata, d'Orbigny ........... 42

- flora, Pfeiffer ........... 40

- Folini, Morelet. . . . . . . . 16

- fulica, Férussac ........... . 76

- liylephila, d'orbigny ......... 42

- lbuensis, Pfeiffer............ 18

- Jenynsi, il. . . . . . . . . . 19

- Moyobambensis, Moricand. . . . . . . . . 40

- nozambicensis, Pfeiffer. ......... 17

- il. Smith .......... 17

- mulata, Gould ............ 4. 4 
Helix Nyassana, Smith........... 17

- omalomorpha, d'0rbigny ......... 40

- orbicula, id. ........ 40

- panthera, Férussac. . . . . . . . . 75

- Paraguayana, Pfeiffer ... . . . . . 42

- perspectiva, Wagner ......... . 44

- plicatula, Pfeiffer. .......... 17

— skiaphila, d’orbigny ......... 41

- spirorbis, Deshayes. . . . . . . . . . 41

- Spixiana, [feiffer. . . . . . . . . . 44

- Thomasi, $i d . \ldots . . . . . . .41$

— trochilioides, d'0rbigny......... 40

- ustulata, Férussac . . . . . . . . . 80

— Vitrina, Wagner.......... . 40

- Wagneri, Pfeiffer. . . . . . . . . . 45

Hyalina argentina, Strobel . . . . . . . . 4 41

- omalomorpha, Albers. . . . . . . 40

Iridina Wahlbergi, Krauss . . . . . . . 196;

LANISTES, Denys de Montfort, 1810 . . . . . . 173

Lanistes affinis, Smith . . . . . . . . 173

- Bernardianus, Pfeiffel ........ 17i

- ciliatus, Martens. . . . . . . . . 177

- ellipticus, Pfeiffer.......... 173

- Guinaicus, id. ........ 175

- Libycus, var., id. ......... 173

- Nyassanus, Dohrn ........ 180

- olivaceus, Pfeiffer ......... 172

- id. var. procerus, Pfeiffer. ..... 172

- id. var. ambigua, id. ...... 173

- Olivieri, Denys de Montfort. . . . . . 179

- ovum, Dohrn.......... 174

- id, var. elatior, Pfeiffer....... 173

- purpureus, Dohrn . . . . . . . 170

- solidus, Smith. . . . . . . . 175

- Zambesianus, Furtado . . . . . . . 173

LEDOULX1A, Bourguignat, 188ว ........ 24 
Pages.

Ledoulxia albopicta, Bourguignat ........ 24

- Alfieriana, Soleillet. . . . . . . . 24

- formosa, Bourguignat. ....... 2o

- insignis, $\quad i d . \quad \ldots . . . .26$

- megastoma, id. . . . . . . 26

- pyramidaca, id. ........ 2.

- Inizonata, ill. . . . . . . 27

LEROYA, Grandidier, 1887 . . . . . . 180

I.croya Bourguignati, Grandidier ........ $\$ 80$

一 Charmetanli, id. ........ 180

I.IIICOLARIA, Schumucker, 1817 ...... . 101

Limicolaria Bourgnignati, Grandidier . . . . . . 91

- Bridouxi, id. ....... 103

- Charbonnieri, Bourguignat ... 102, 10 f

- Coulboisi, id. .... 103, 106

- Dromauxi, id. $\quad . . . \quad 103,107$

- Giraudi, id. . . . . . 104

- Kraussi, H. et A. Adams. . . . . . . 95

- lamellosa, Bourguignat ..... 104, 109

- Martensiana, Martens......... 103

- id. Crasse ........ 104

- megalæa, Bourguignat ..... 102, 103

— nilotica, Pfeiffer ......... 89

- id. (pars), Pfeilfer. . . . . . 95

- rectistrigata, Grandidier. . . . . . 103

- sepulcralis, Bourguignat. . . . 103, 108

— Spekiana, Grandidier ....... 102

一 subconica, Martens . . . . . . . . . 99

LINNEd ' Lemarck, 1799 et 1801 . . . . . . . . 15t

Limnæa africana, Ruppell . . . . . . . . . 157

- De Baizei, Bourguignat ........ 157

- Cameroni, il. ....... 157

- liynganica, id. . . . . . 158

- truncatula, Gorpil ......... 152

1. Voir, à la page 155 , la liste des Limnées africaines. 
Pages.

Limnca Zanziharica, Bonrguignat. . . . . . . . 1 aั8

MAIZANIA, Bourguignat, $1889 . . . . . .148$

Maizania olivacea, Bourguignat ....... 149

MARCONIA, Bourguignat, 18:9 . . . . . . 133

Marconia enneoides, Bourguignat. . . . . . . 137

- gibbosa, id. ........ 137

- lata. il. . . . . . . 136

- recta, $\quad i d . \quad \ldots . . . . . .138$

- vitrea, $i d . \quad \ldots . . . . .136$

Margaritana, Pfeifferiana, Bernardi . . . . . . 196

Martensia Mozambicensis, Semper. . . . . . . 18

MELADOMUS, Swainson, 1840........ 169

Meladomus affinis, Bourguignat...... 175

- Alexandri, $\quad i d . \quad \ldots . . .177$

- ambiguus, id. ...... 172

- Bernardianus, id. ...... 176

- Bloyeti, id. . . . . . 171

- Boltenianıs, id. ..... 178

- ciliatus, id. . . . . . 177

- Deguerryanus, il. ...... 17\%

- Duveyrierianus, id. . . . . . 179

- elatior, id. ...... 173

- ellipticus, $\quad i d . \quad \ldots . . .173$

- Grasseti, $\quad$ id. ....... 172

- Guinaicus, $i d . \quad \ldots . . .175$

- holostoma, iil. . . . . . $1 ; 6$

- intortas, $i d . \quad \ldots . . . .174$

- Jouberti, $\quad i d . \quad$...... 174

-. Letourneuxi, $\quad i d . \quad$. . . . . . 179

- Libycus, $\quad i d$. . . . . . 176

- niloticus, id. ...... 176

- nitidissimus, $\quad i d$. . . . . . . 171

- Nyassanus, $i d . \quad$. . . . . 179

- olivaceus, H. et A. Adams . . . . . 170

- id. Swainon......... 172

- ovum, Bourgnignat........ 173 
Meladomus Pfeifferi, Bourguignat. . . . . . . 173

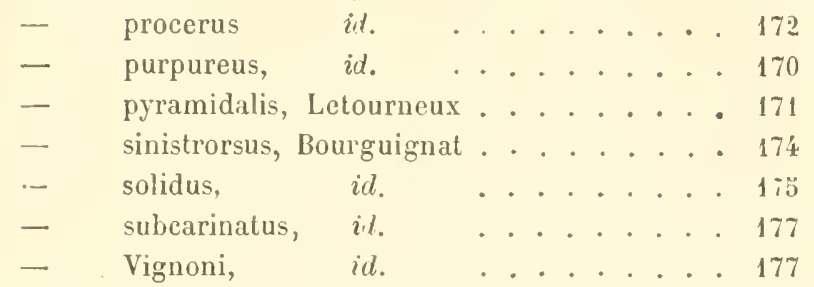

MELANIA, Lamarck, 1799 et 1801 . . . . . . 182

Melania crenularis, Deshayes. . . . . . . 183

- id. Martens. . . . . . . 183

- fasciolata, Lamarck. . . . . . . . 182

- Inhambanica, Martens. . . . . . . . 183

- tuberculata, Bourguignat . . . . . 182

- Zanguebarensis, Pelit . . . . . . . 164

MOARlA, Chaper, 1885. ........... 14

Moaria calabarica, Bourguignat. . . . . . . 14

- Chaperiana, id. . . . . . . 14

- conica, Chaper. . . . . . . . . 14

— talcosa, Bourguignat ......... 14

Nanina aglypta, IIartens . . . . . . . . . 16

— Jenynsi, Gray ........... 19

- Mozambicensis, var. albopicta, Martens . . . . 24

- plicatula, Martens. ......... 17

- pyramidæa, $i d . \quad \ldots . . . . .25$

Nerita tuberculata, Müller. . . . . . . . $1 \times 2$

OEROPE, Albers, 1860.............. 11

Otopoma obtusum, Pfeiffer.......... 147

- Perrieri, Bourguignat. . . . . . . 144

- Poirieri, id. ......... 144

PACHNODUS, Albers, $1 \& 60 \ldots . . . . .64$

Pachnodus Leroyi, Bourguignat. . . . . . 65

- Natalensis, $i d . \quad \ldots . . . . .65$

- Rochebrunianus, $i d$. . . . . . . . 59

- sesamorum, Ancey. . . . . . . . 66

- spadiceus, Menke ......... 6 . . 6 . 
Pachnodus Spekei, Bourguignat. ..... Pages,

- Vescoi, id. ......... 59

Paludina olivacea, Sowerby . . . . . . . 172

- sinistrorsa, Lea. . . . . . . 174

Paludomus africanus, Martens . . . . . . . 164

- Ajanensis, Morelet......... 165

- exaratus, Martens. ......... 16t

PARACHATINA, Bourguignat, 1889....... 73

Passamaella, Pfeiffer, 1877......... 128

PIIARAONIA, Bourguignat, 1880........ 191

Pharaonia Bellamyi, Jousseaume . . . . . . 192

Bourguignati, Rochebrune . . . . . 192

PHYSOHSIS 1, Krauss, 1848. . . . . . . 159

Physopsis Bloyeti, Bourguignat . . . . . . 160

- I.eroyi, Grandidier. . . . . . . 160

- ovoidea, Bourguignat. ........ 160

- præclara, id. . . . . . . 160

- Soleilleti, id. ....... 160

- Stanleyana, il. ....... 1 1i0

PINTOA, Bourguignat, 1889......... 80

PLOTlA, Bolten, 1798. . . . . . . . 18'

Plotia Bloyeti, Bourguignat. . . . . . . 18i;

- Leroyi, $i d . \quad \ldots . . . . . .185$

PTYCOTREMA, Nærch, 1802 . . . . . . 124

Ptycotrema assinicum, Bourguignat ....... 125

- cyanostoma, i.l. ...... 124

- Elimense, id. . . . . . 12J

- Guinaica, id. . . . . . 12t

- Guineensis, Morch ......... 12t

- mucronalum, Bourguignat . . . . . . 12f

- ringens, id. ....... 124

- Treichi, $\quad i d . \quad \ldots . . . . .125$

Pupa bicolor, Hutton. . . . . . . . . . . . . 123

- Ceylanica, Pfeiffer ... ........ 123

1. Voir, à la page 159 , la liste des Physopsis africaines. 
Pupa grandis, Pfeiffer . . . . . . . . . . . 140

- intermedia, Morelet. . . . . . . . . . 140

- minor, id. ............. 141

- Pirrieri, Pfeiffer . . . . . . . . . . 123

RACIHS, Albers, 1850 . . . . . . . . . . . 55

Rachis Bloyeti, Bourguignat. . . . . . . . . . 60

- Braunsi, Martens. . . . . . . . . . . 59

- Cameroni, Bourguignat. . . . . . . . . 58

- catenatus, Martens ........... . 60

- elongatulus, Bourguignat. . . . . . . . . 63

- Ferussaci, Pfeiffer ........... . 70

_ Hildebrandti, Martens. . . . . . . . . . 59

- Jouberti, Bourguignat.......... 61

- jaclistoma, $\quad i d$. . . . . . . . 62

- pallens, Albers . . . . . . . . . . . . 8

- picturatus, Bourguiguat. . . . . . . . . 60

- rhodotænia, Martens . . . . . . . . . 60

- Rochebrunianus, Bourguignat. . . . . . . . 59

- Spekei, ir. . . . . . . 59

— stictus, Martens. . . . . . . . . . . 60

— subconicus, Bourguignal . . . . . . . . . $\$ 9$

- valiolosa, Pfeiffer. . . . . . . . . . . 70

- Vescoi, Bourguignat . . . . . . . . . 59

RACHISELLUS, Bourguignat, 1889. . . . . . . . . 68

Rachisellus Burtoi, Bourguignat . . . . . . . . 6!

- Furussaci, id. ........ $i 0$

- Ledoulxi, $i d$. . . . . . . 70

- punctalus, il. $\quad$......... . 69

- variolosus, il. ........ 70

ROCIFERLNIA, Bo rrguignat, 188t. . . . . . . . 143

Rochebrunia Delmaresi, Ancey . . . . . . . . 146

- guillainopsis, Bourguignat. . . . . 146

- insularis, $t d$. . . . . 146

- Letourneuxi, Incey . . . . . . . 132

- obtusa, Bourguignat. ..... . 147

- zanguebarica, id. . . . . . . 147 
Pages.

SCOL.0D0NTA, Doring, 1875 . . . . . . . . . 40

Scolodonta achtephila, Bourguignat. . . . . . 42

- ammonoceras, ill. ...... 41

- argentina, id. ...... 41

- Bounobæna, $i d . \quad . . . . .42$

- chalicophila, $i d$. . . . . 41

- hylephila, $\quad$ id. . . . . . 42

- mutata, id. ...... 42

- Paraguayana, il. ...... 42

- Semperi, id. ...... 41

-. skiaphila, id. ...... 41

- spirorbis, $\quad i d . \quad \ldots . . . .41$

- Thomasi, id. ...... 41

SERPAA, Bourguignat, 1887. . . . . . . . 74, 8 כ

Serpac Dammarensis, Bourguignat . . . . . . . . 85

- Hortensix, id. . . . . . . 85

- Knori, id. ........ 8 ă

- obesa, il. $\quad$. . . . . . . 86

- Pintoi, id. $\quad$. . . . . . . . 86

- varicosa, $\quad i d$. . . . . . . 86

SITALA, H. Adams, 180ั6 . . . . . . . . . . . . 13

Sitala membranacea, Bourguignat . . . . . . . . 13

- Raffrayi, id. . . . . . . . 13

- trochulus, Pollonera . . . . . . . . . 13

Solarium candidum, Spix . . . . . . . . . . . 44

Spatha Bourguignati, Ancey . . . . . . . . . . 197

- Nyassaensis, Lea . . . . . . . . . . . 196

- sinuata, Martens. . . . . . . . . . . . 196

- Wahlbergi, id. . . . . . . . . . . 196

SPATHELLA, Bourguignat, 188ă. . . . . . . . . . 19 วั

Spathella Adansoni, Jousseamme. . . . . . . . . 196

- Bloyeti, Bourguignat . . . . . . . . 198

- Bourguignati, Ancey . . . . . . . . 197

- Nyassana, Bourguignal . . . . . . . . 196

- Pelersi, $\quad i d . \quad$. . . . . . . . 197

- Pfeifferiana, id. . . . . . . . 196 
Pages.

Spallsella Protchei, Rochebrune. . . . . . . . 196

- sinuata, Bourguignat........ 196

- spathuliformis, $i d$. . . . . . . 199

- Wahlbergi, $i d . \quad \ldots . . . . .196$

STENOGYRA Shuttleworth, 1834......... 110

Stenogyra Grandidieriana, Bourguignat ...... 111

- Leroyi id. . . . . . 110

STREPTAXIS ${ }^{1}$ Gray, 1837. . . . . . . . 130

Streptaxis alveus, Philippi. . . . . . . . . . 47

- apertus, Martens. ......... 47

- Crossei, Pfeiffer......... 46

- discus, $i d . \ldots . . . . . . .44$

- enneoides, Martens. . . . . . . 137

- gigas, Smith ......... 38

- intermedius, Albers ......... 4 t

- Paivana, Pfeiffer........... 47

- Semperi, Dœring ......... 41

- uberiformis, Pfeiffer......... 44

- Wagneri, var. $i d . \quad . . . . . . .444$

SUBULINA, Beck, 1838 . . . . . . . . . . 113

Subulina cylindracea, Bourguignat . . . . . 115

- intermedia, Gibbons. ......... 11'

- Jouberti, Bourguignat. ........ 115

- lenta, Smith .......... 11t

- Mabilliana, Bourguignat. ....... 11'

TAPSId ${ }^{2}$, Albers, 1860.............. 12

Tapsia Leroyi, Grandidier .......... 12

TAYLORIA, Bourguignat, $1889 \ldots . . . .3 s$

Tayloria Jouberti, Bourguignat . . . . . . 39, 47

- ventrosa, $i$. . ......... 39

TlARA, Bolten, 1798........... 183

Tiara crenularis, H. et A. Adams . . . . . . 183

1. Voir, aux pages 129 et 130 , la liste des Espèces de re genre.

2. Voir, ì la page 12, la liste des Espèces de ce genre. 
Tiara Vouamica, Bourguignat. . . . . . . . 183

Tiaropsis, Brot, 1874. . . . . . . . 183

ThIPACHATINA, Bourguignat, 1889 ....... 73

Trochomorpha Folini, Pfeiffer. . . . . . . . 16

TROCHONANINA, Mousson, 1\$69 ....... 15

Trochonanina Adansoniæ, Bourguignat. . . . . . 16

- aglypla, id. ...... 16

- Anceyi, ill. . . . . . 20

- Bloyeti, id. ....... 21

- Folini, id. ...... 10

- lbuensis, Martens. . . . . . . 18

- Jenynsi, $i d . \quad . . . . . . . .19$

— Leroyi, Bourguignat. . . . . . . 18

- Mozambicensis, Mousson. ...... 17

- nyassana, Bourguignat ....... 17

— percarinata, Martens ....... 19

- plicatula, Pfeiffer. . . . . . . 17

- pyramidata, Martens ........ 25

- Smithi, Bourguignat ....... 17

- tumidula, Martens ....... 16

UNI0, Philipsson, 1788........... 192

Unio Billotianus, Charmes ........ 19'

- Dumesnilianus, id. ........ 193

- Essoensis, Chaper . . . . . . . . . 192

- euphymus, Charmes........... 194

- Juliani, Rang . . . . . . . . . . . 192

- Ledoulxianus, Charmes. ......... 194

— Mossambicensis, Peters. . . . . . . . 193

- ratidotus, Charmes. ......... 193

VALVATA, Muller, $1774 \ldots . . . . . . . .9187$

Valvata ægyptiaca, Bourguignat. . . . . . . 187

- callista, $\quad i d . \quad$. . . . . . . . 187

— Delevieleusæ, Hagenmüller . . . . . . . 187

- exilis, Paladilhe ........... 187

- Hagenmölleri, Bourguignat . . . . . . 187

kliedivialis, Letourneux . . . . . . . 187 
Pages.

Valvata Letourneuxi, Bourguignat. . . . . . . 188

- nilotica, Jickeli. . . . . . . . . 188

— Petrettinii, Letourneux ........ 189

- Pharaonum, Bourguignat . . . . . . 188

- planorbulina, Paladilhe . . . . . . 188

- planulata, Bourguignat ........ 188

- Revoili, $\quad i d . \quad \ldots . . . . .189$

- Rothi, il. ........ 188

- Saulcyi, id. ......... 188

- Schweinfurthi, $i d$. . . . . . . 188

- Scioana, Pollonera ........ 188

- syriaca, Bourguignat . . . . . . . 188

VIVIPARA, Lamarck, 1809 . . . . . . . 162

Vivipara capillata, Frauenfeld. . . . . . . 162

— Jeffreyssi, $i d . \quad$. . . . . . . 163

- Robertsoni, id. ......... 162

ZINGIS, Martens, $1878 \ldots . . . . . .22$

Zingis radiolata, Martens. .......... 22

Zonites ventrosa, Taylor ......... . 39

F I N 

(i) $<0$

(b) $<$

$\Leftrightarrow 6$

\& $\quad 6$

$64<$ 



Moll Afriq. équat.

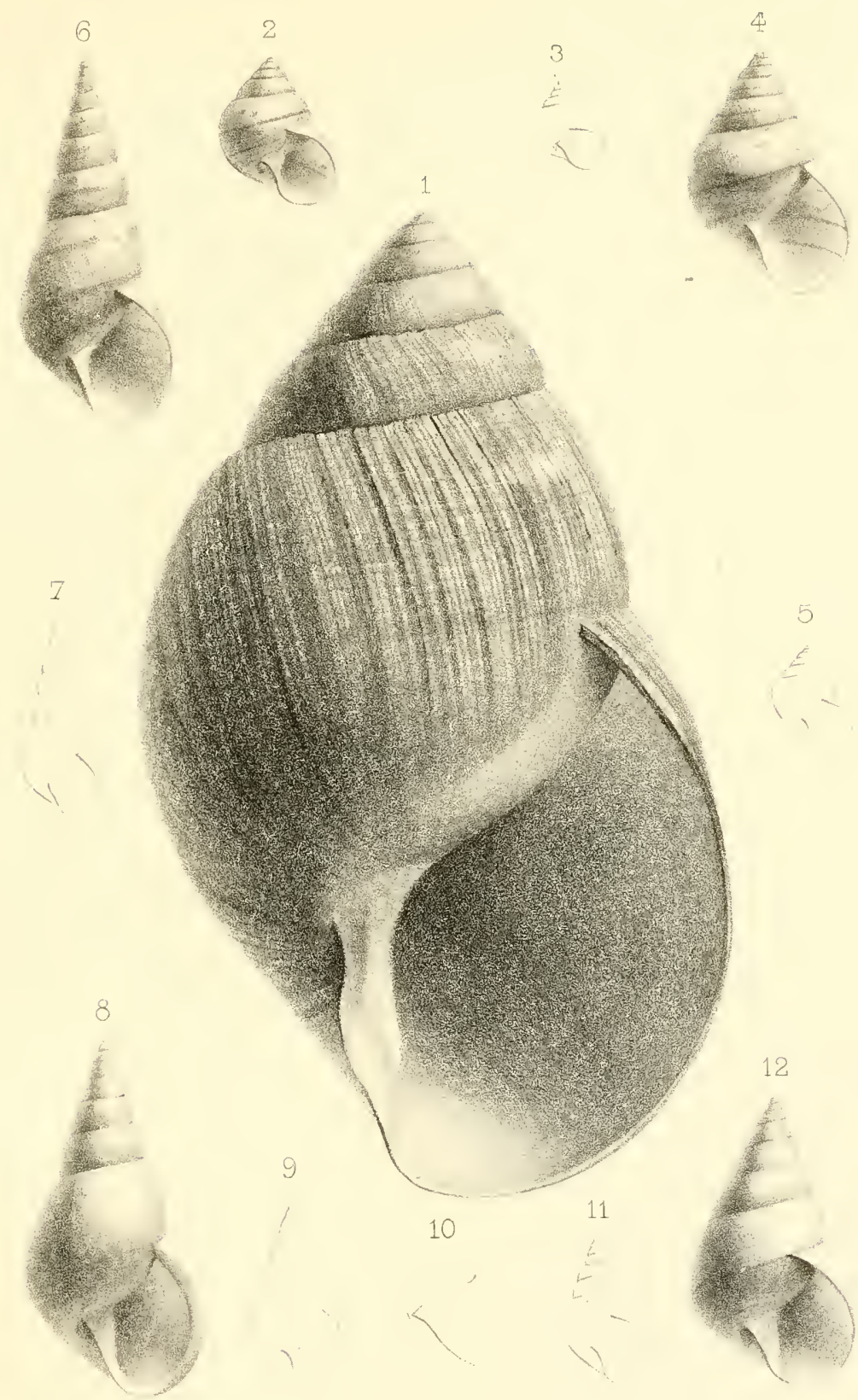

A. de Vaux-Bidon del

Imp. Edouard Bry, Paris.

1. Burtoa sebasmia, 2-3. Pachnodus Sesamorum,

4-5. Pach. Leroyi, 6-7. Rachis elongatulus,

8-9. Rachis pachistoma, 10-12. Rachis Jouberti. 


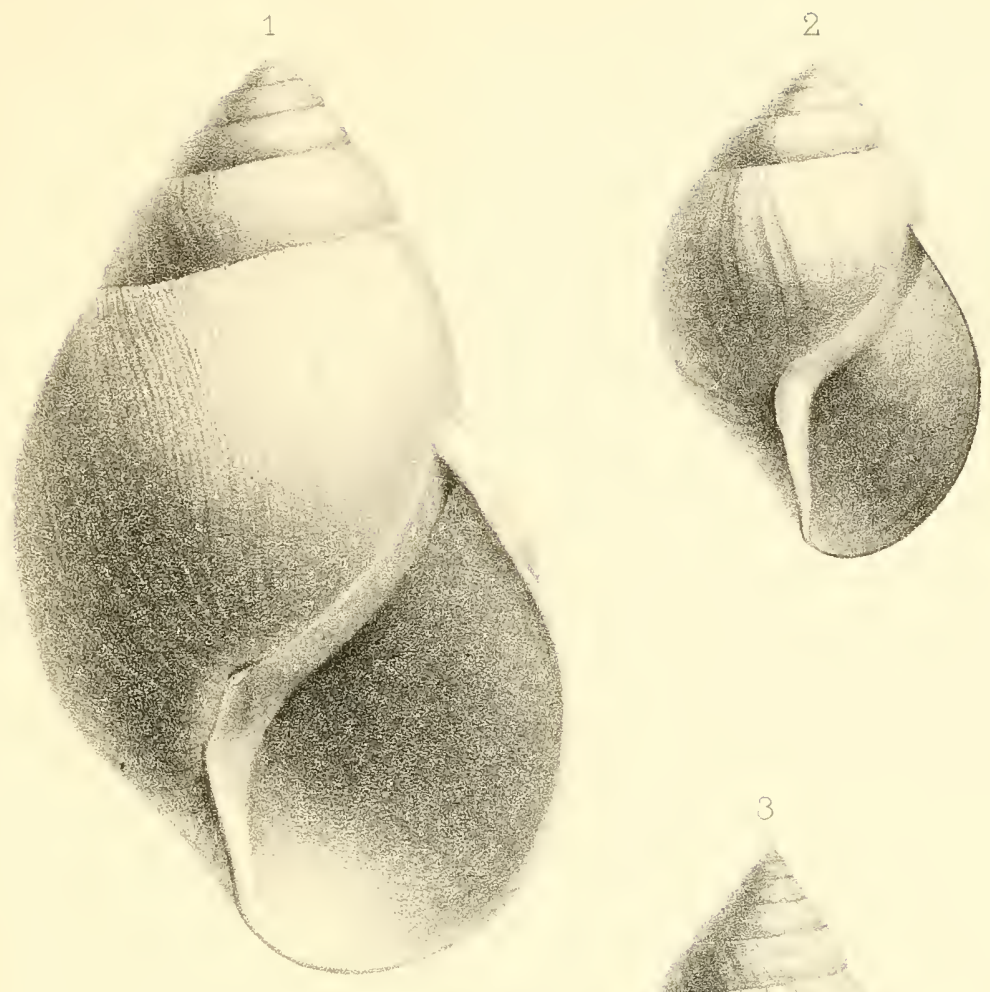

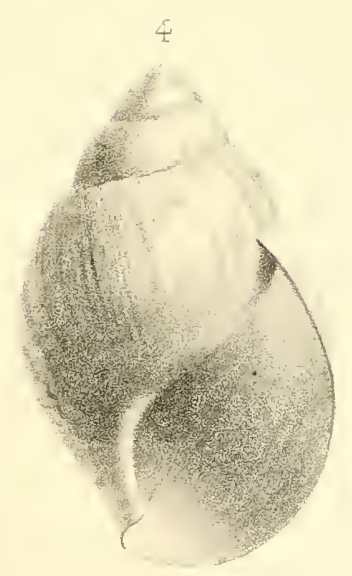

1. Ie vaux-Bidon del.

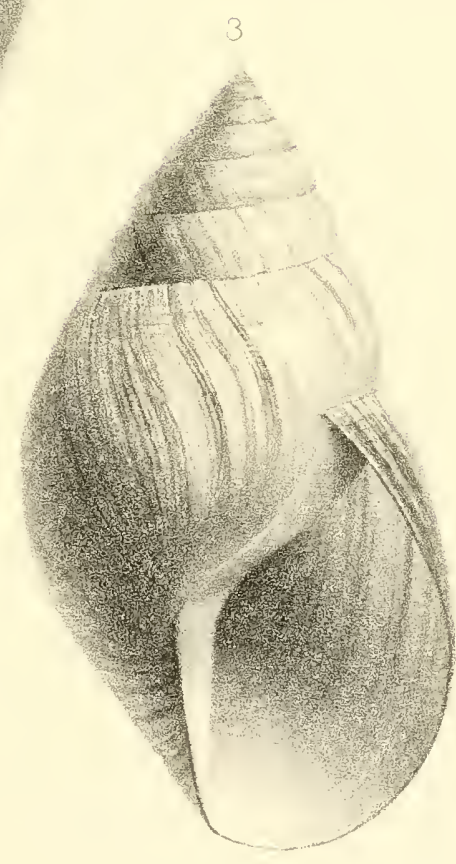

Imp. Edouard Bry, Paris
1. Burtoa Reymondı, 2. Burtoa Lavigeriana,
3. Burtoa Bridouxiana, 4. Serpoea Pintor 



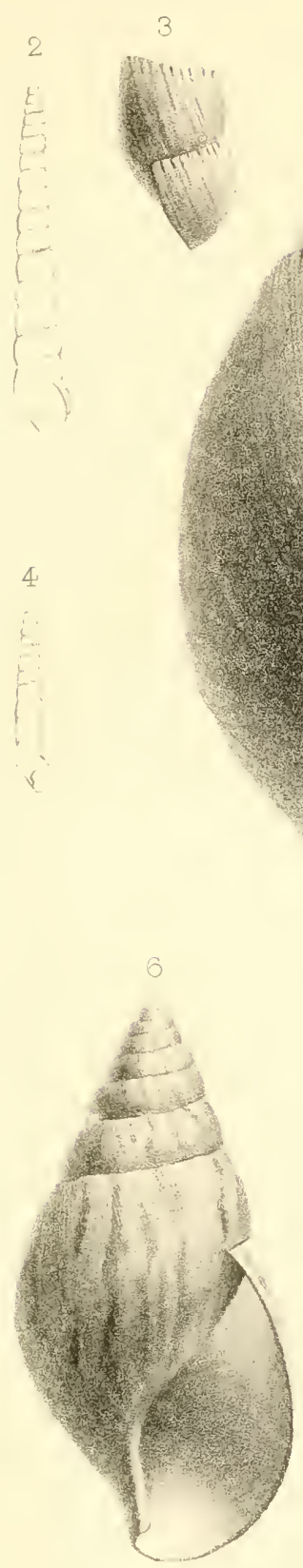

A de Vaux-Bidon del.
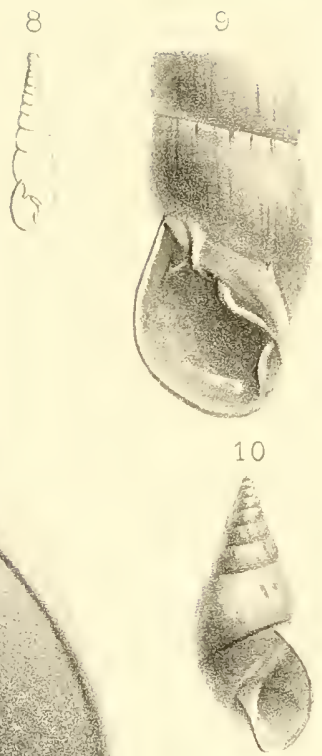

11
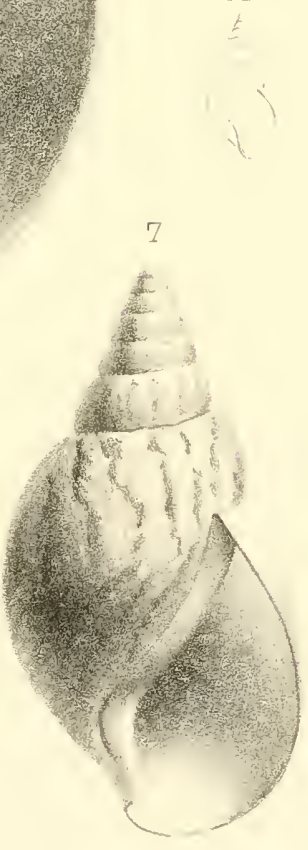

Imp. Edouard Bry, Par's

1. Burtopsis Giraudi, 2-3. Subulina cylindracea,

4. Sub. Jouberti. 5. Sub. lenta, 5. Achatina Randabeli, 7. Ach. arctespirata, 8-9 Clausilia Giraudi 10-11. Rachisellus Ledoulxi. 


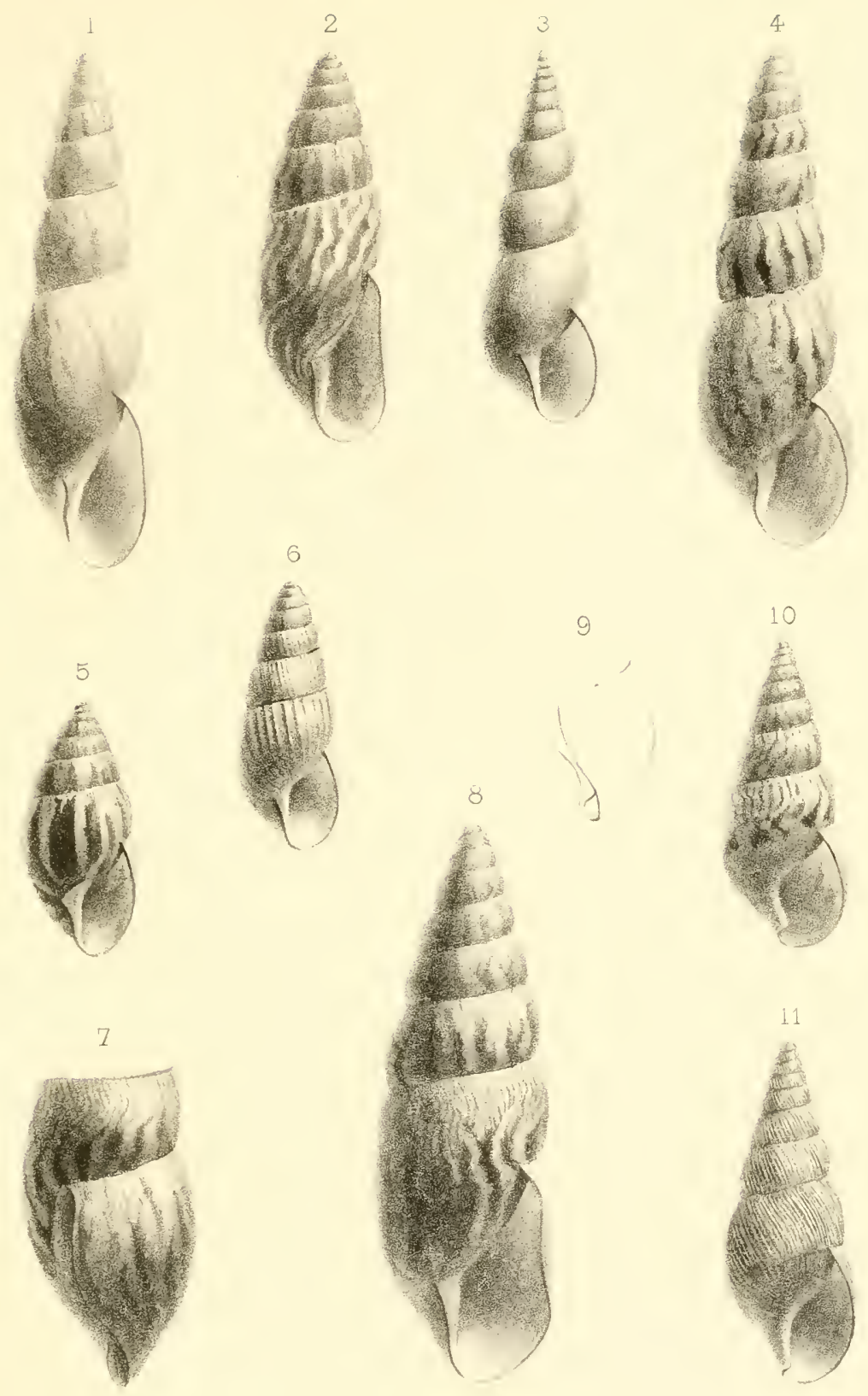

1. Limicolarıa Coulboisı. 2. Limic sepulcralis, 3. Limic Dromauxı, 4. Limic megaloea, 5. Limic. Giraudi.

6. Limic lamellosa, 7-8. Limic. Charbonnieri.

9-10 Stenogyra Grandideriana. 11. Sten Leroyi 


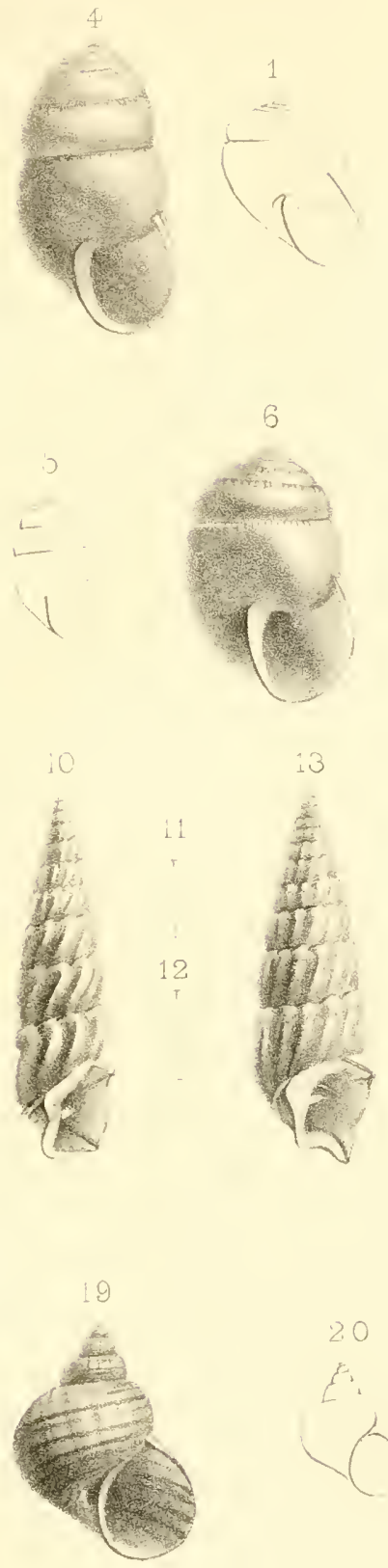

A de Vux-Bidon del
3
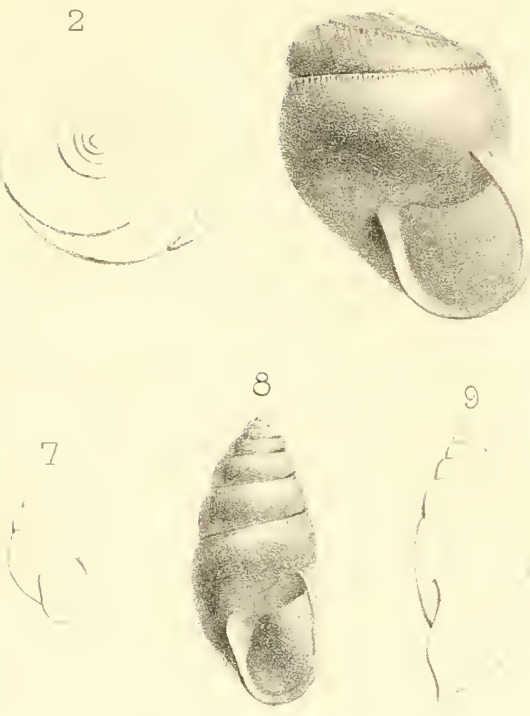

9

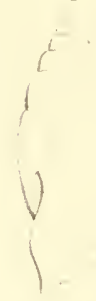

16

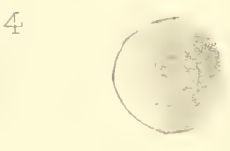

17

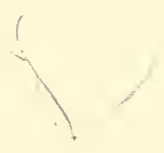

2.1

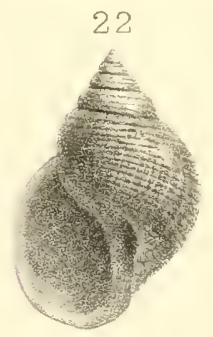

Inp Edor.tard Bry Paris

1-3. Gonaxis Bloyeti, 4-5. Marconia recta, 6-7. Marc.

gibbosa, 8-9. Edentulina Grandidieri, 10-11. Anceya admirabilis, 12-13. Anc. Giraudi, 14-18. Maizania olivacea, 19-20. Cyclostoma Leroyi, 21-22. Leroya Charmetantı. 


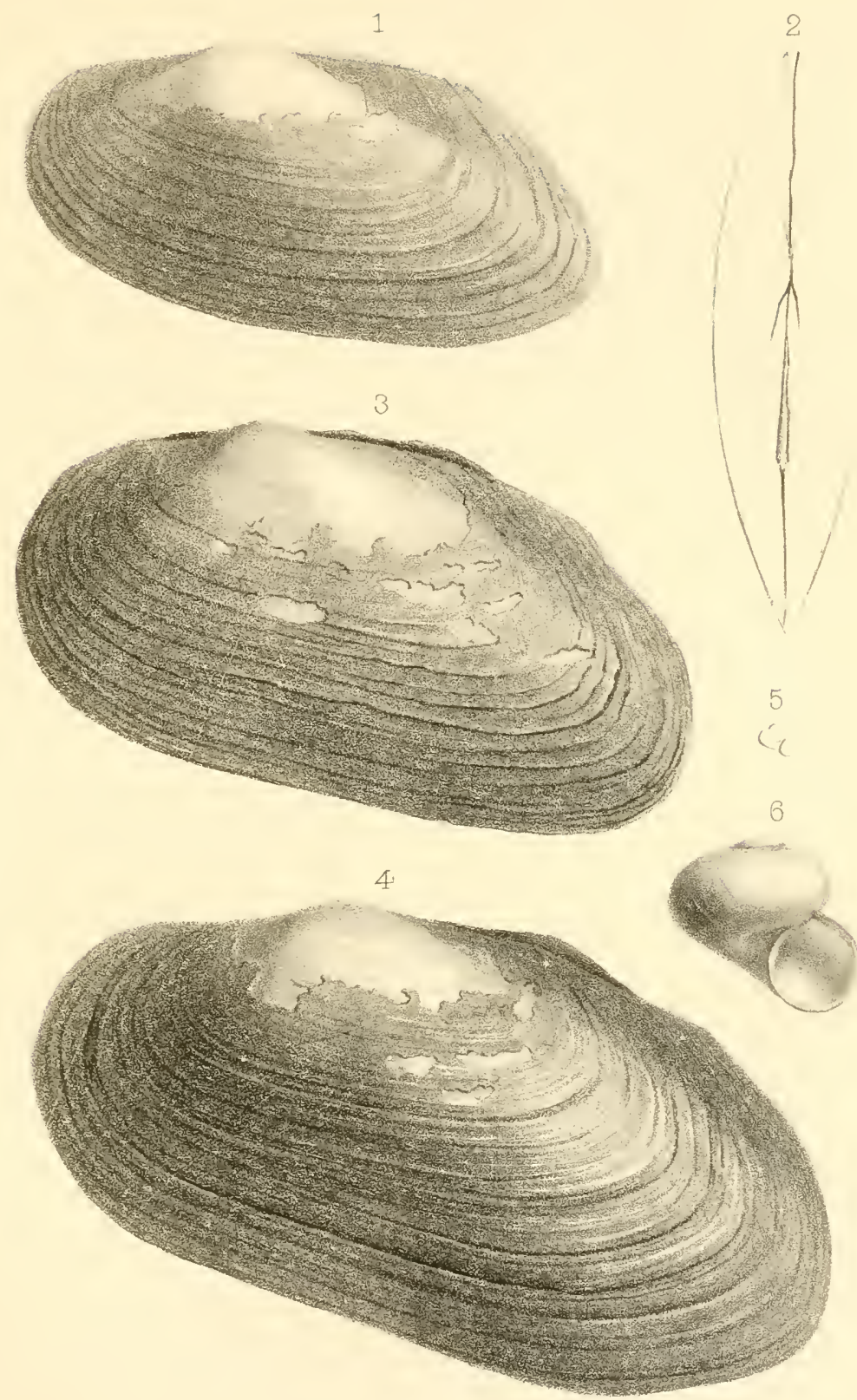

A. de Vaux-Bidnn del.

Imp Edouard Bry, Parss

1-2. Spathella Bourguignati, 3. Spathella

Bloyeti, 4. Spathella spathuliformis,

$$
\text { 5-6. Valvata Revoili. }
$$






